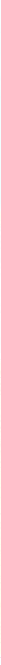

\title{
Self-governance of water resources under climate change: Insights from Cambodia
}

\section{Tum Nhim}

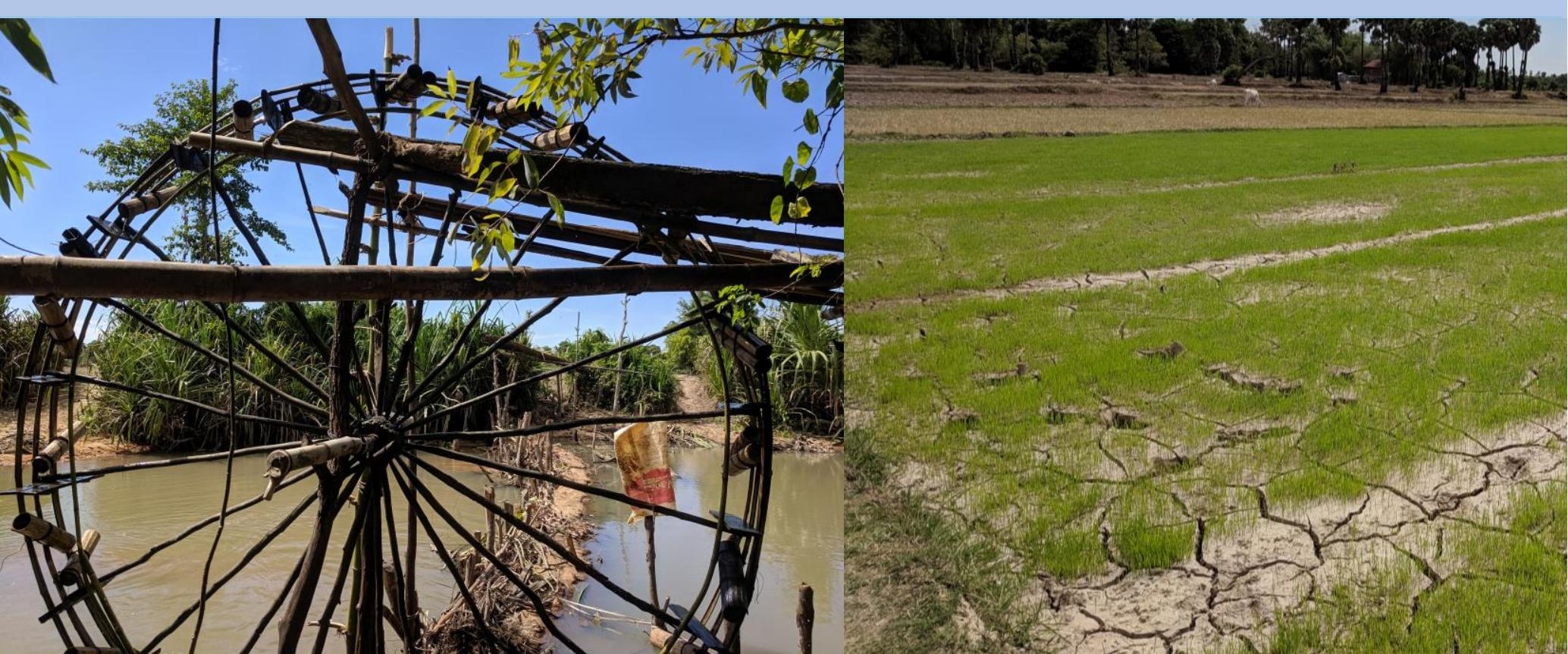




\section{Propositions}

1. The robustness of cooperative arrangements in a self-governing community depends on how well its members can deal with scarcity.

(this thesis)

2. If humans are strongly connected, an informal institution such as social norm is the most obvious solution to a free rider problem.

(this thesis)

3. A combination of agent-based modelling and field experiments can provide more insights into human cooperation than traditional game theory.

4. "Re-search" means systematically search again and again for a justifiable answer until the time and resources are exhausted.

5. Social sciences are not free from bias unless the mind of scientists is in equanimity.

6. Behavioral scientists will never fully understand human behavior unless their own mind becomes the subject of experimentation.

7. To eliminate mental stress, one needs to fully align expectation with reality.

8. The most efficient way to enhance memory naturally is to always tell the truth.

Propositions to the thesis, entitled

Self-governance of water resources under climate change: Insights from Cambodia

Tum Nhim,

Wageningen, 1 July 2021 


\section{Self-governance of water resources under climate change: Insights from Cambodia}

Tum Nhim 


\section{Thesis committee}

\section{Promotor}

DrX Zhu

Associate Professor, Environmental Economics and Natural Resources Group Wageningen University \& Research

\section{Co-promotor}

Dr AP Richter

Associate Professor, Environmental Economics and Natural Resources Group Wageningen University \& Research

\section{Other members}

Prof. Dr GJ Hofstede, Wageningen University \& Research

Dr KH Pham Do, Massey University, Palmerston North, New Zealand

Dr GAK van Voorn, Wageningen University \& Research

Dr J.-C. Diepart, Gembloux Agro-Bio Tech, University of Liège, Belgium

This research was conducted under the auspices of the Graduate School for SocioEconomic and Natural Sciences of the Environment (SENSE) 


\title{
Self-governance of water resources under climate change: Insights from Cambodia
}

\author{
Tum Nhim
}

Thesis

submitted in fulfilment of the requirements for the degree of doctor at

Wageningen University

by the authority of the Rector Magnficus

Prof. Dr APJ Mol

in the presence of the

Thesis Committee appointed by the Academic Board

to be defended in public

on Thursday 1 July 2021

at 11 a.m. in the Aula. 
Tum Nhim

Self-governance of water resources under climate change: Insights from Cambodia, 146 pages.

$\mathrm{PhD}$ thesis, Wageningen University, Wageningen, NL (2021)

With references, with summary in English

ISBN 978-94-6395-792-2

DOI https://doi.org/10.18174/545818 


\section{Acknowledgements}

What a long and exciting journey it has been! I began this journey alone, but over time many more people join in to make this journey unforgettable. Without supports from all of them, this journey couldn't have been successful and memorable.

First of all, I am deeply indebted to my supervisor, Andries. He is extraordinary at both personal and professional levels. He is very kind, supportive and encouraging. Instead of suggesting or commanding, he helps me to paint the whole picture of my doctoral research in a way that this thesis is purely emerged out of my personal interest while scientifically relevant. Whenever I got stuck with something personally and academically, the first person I talked to is Andries. I am probably one of the very lucky doctoral students who have chance to work with him. I am very grateful for the support of my co-supervisor and promotor, Xueqin. She is very helpful and supportive. Big thanks to Xueqin and Ekko for giving me the chance to join ENR, and being part of this exciting group.

ENR is a lovely and stimulating group. Beside academic supports, social interactions, coffee breaks, group outing, and other social supports are all incredible. Without all of these, my $\mathrm{PhD}$ journey is undoubtedly tough and lonely. Immense supports from Wil and Gre on academic and living arrangements are touching for me. I am very thankful to all ENR colleagues for spending time with me chitchatting and discussing about sciences and life. Special thanks to people at the Center for Khmer Studies and at IIASA, especially Matthias and Nikita, for providing supports and stimulating environment for me to work on one of the chapters.

A warm and sincere thank to my dear friends and colleagues Anna, Esther, and Sanmitra. I never thought I could have such amazing friends like you. Anna is very kind and empathetic. Sanmitra is someone who I can just sit with and talk about anything personally. I won't forget our amazing fieldwork experience in Cambodia, Esther. It was incredibly challenging since it was the first ever field experiment we run. Many more authentic foods and fruits are for you to try out in Cambodia, and you are always welcome at any time to come again for personal or field visits.

Finally, a heartfelt thanks to my lovely wife Saem. I am the luckiest man to share this journey with you. Without your support, this $\mathrm{PhD}$ journey could not have continued till now. You took care of the kids, Tika and Suosdey, and acted as a father while I was away. Tika and Suosdey 
is the greatest source of motivation and is the main reason why I could overcome any challenges during difficult times. My parents and brothers and sister provide me a real strength to finish this $\mathrm{PhD}$. Being the first person in the family to ever pursue a $\mathrm{PhD}$ is what they are proud of, and my determination to make their wish come true is the reason why this $\mathrm{PhD}$ journey is successful.

My sincere thanks to all of you! 


\section{Table of Contents}

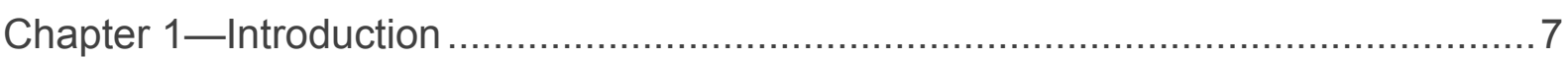

Chapter 2-The resilience of social norms of cooperation under resource scarcity and inequality - an agent-based model on sharing water over two harvesting seasons

Chapter 3-Path dependencies and institutional traps in water governance: Evidence from Cambodia.

Chapter 4-Multi-level water self-governance-cooperation within and between communities in Cambodia

Chapter 5-Voting on costly institutions in public goods: Experimental evidence from Cambodia

Chapter 6-Synthesis

Summary 136 



\section{Chapter 1-Introduction}

\subsection{Background}

Globally, water governance is facing several challenges including increasing scarcity due to growing demand as well as climate change (Haddeland et al. 2014; Elliott et al. 2014). While climate change directly impacts water supply by decreasing mean water availability (Schewe et al. 2014; Haddeland et al. 2014) and increasing water's variability in time and space (Jaeger et al. 2017), human behavior of water use shapes the demand and sustainability (Schill et al. 2019). Across regions, human's water use in the upstream of a river basin exacerbates water scarcity in the downstream (Veldkamp et al. 2017), highlighting the importance of coordinated human behavior in managing water scarcity. Since the interactions between human behavior and water systems are complex (Wada et al. 2017), understanding how its dynamics unfold remains a challenge (Schill et al. 2019). This is especially the case when water is managed as a common-pool resource, in which water consumption by one user is not available to others and excluding others from using it is too costly or difficult. Common-pool resources are typically considered as part of a so-called social-ecological system (SES), where the interactions between the resource (water), the biophysical system, resource users, and the governing institutions are complex, non-linear, and exhibit thresholds (Ostrom 2009; Liu et al. 2007). Given the complexity of the system, this thesis mainly focuses on small-scale irrigation systems, which can be considered as a SES. At the heart of such a system lies the capacity to self-organize for collective action and cooperation among agents, which is shaped by the embedded institutional arrangements, and pivotal in determining the long-term sustainability or resilience with regard to drivers, such as climate change (Yu et al. 2015; Anderies et al. 2004; Ostrom 2009).

This thesis analyzes under what conditions, and with which institutional mechanisms cooperative arrangements can be established and maintained under climate change. I focus on a small-scale social-ecological system (SES), namely an irrigation system which consists of the resource (water), water users (farmers), and the governance system (informal and formal institutions) (Fig. 1). Farmers interact with the resource system by harvesting the water for irrigation and by investing in infrastructure maintenance. They may overharvest the water for personal benefit or align with the agreed-upon rules or norms that promote collective benefits. Likewise, they may jointly contribute to the infrastructure maintenance so the system generates 
more water for everyone, or freeride on efforts by others. The harvesting and investment decisions are complex and depend on the agents' attributes (inequality, preferences, and experiences), and are shaped by the institutions in place. In some cases, water is managed by local communities following informal institutions such as social norms that prescribe who can take how much water and when. Those social norms are usually enforced through interpersonal communication by means of peer sanctions or reputational concerns. In other cases, water is managed through formal institutions, where written rules are enforced by the local government or a farmer organization.

Obviously, the interactions between the subsystems of water, users, and institutions are complex and may produce emergent outcome that may in turn feedback other parts of the system (Ostrom 2009; Muneepeerakul and Anderies 2020). The resilience of this system and its sustainability depends largely on the self-organizing capacity of the social system for collective action such as cooperation (Schill et al. 2019; Anderies et al. 2004; Perez et al. 2016; Ostrom 2009; Lansing et al. 2017). Ostrom (2009) has identified a set of key variables that may interact with the self-organizing capacity of the system, among which are system productivity (e.g. resource scarcity) and social norms and social capital. This thesis investigates the role of resource scarcity, either exogenously caused by climate change or endogenously, caused by resource users to maintain the water infrastructure (a public good), and its coupling with some key contextual variables through the lens of institutions (Anderies et al. 2004). The key question in this thesis is what kind of institutional configurations facilitate cooperation and can thus accommodate the changes in the face of increasing scarcity due to climate change. To answer the question, I will discuss briefly first the theme, rationale and contribution of each or a combination of chapter(s) and then the methodological approach. 


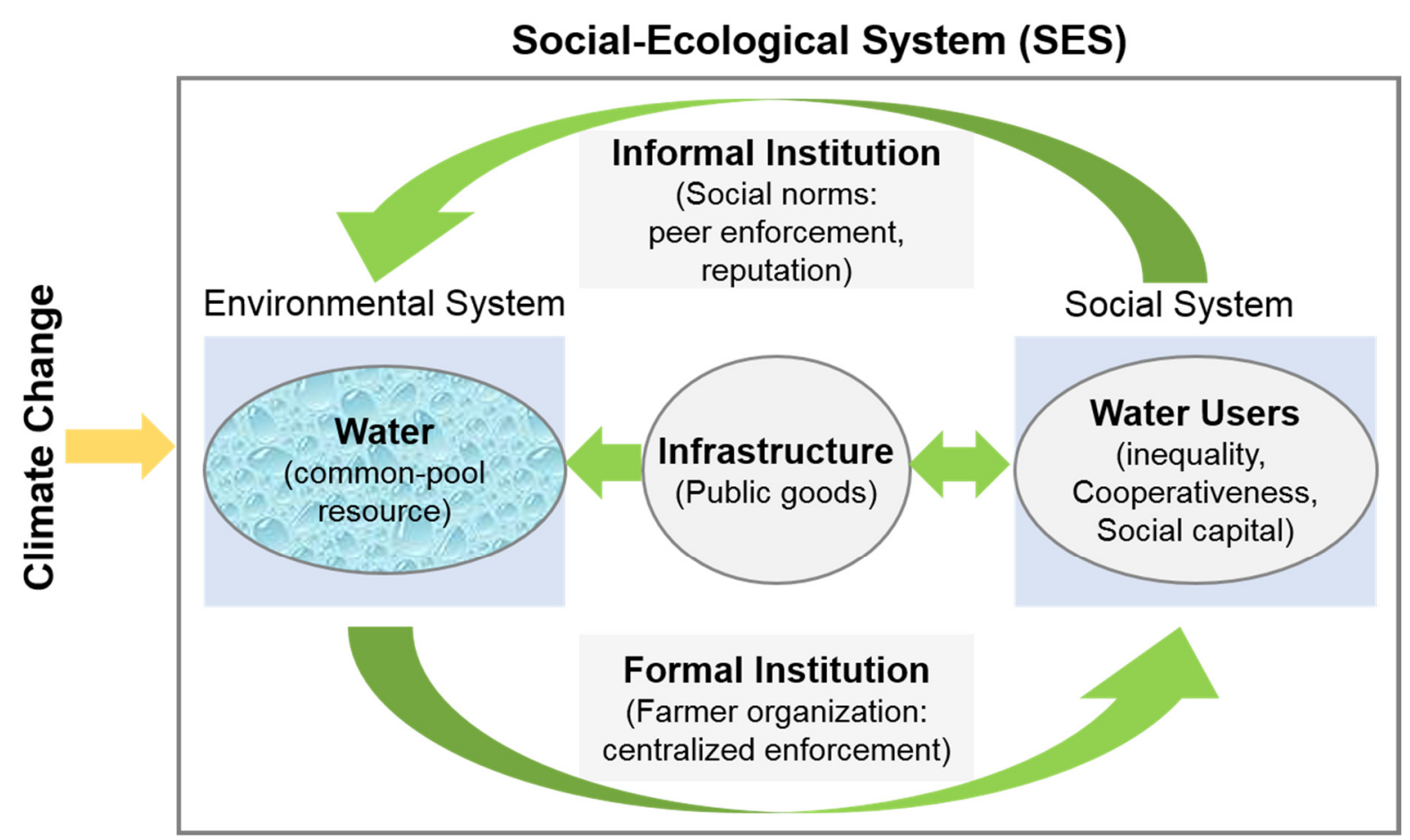

Fig. 1. Thesis's conceptual framework to study the resilience of a small-scale social-ecological system under climate change, with a focus on the role of cooperation in the social system. Climate change directly impacts water supply by increasing scarcity and variability, which translates into changes in economic incentives related to water use. Water users may act cooperatively by restraining water harvest and contributing to maintain the quality of the infrastructure or free-ride on others' effort, considering both immediate economic gains and social consequences. Together, all individual decisions determine the capacity to self-organize and give rise to the co-evolution of social and water systems. Thus individual behaviors are shaped by institutional arrangements embedded as well as the social structure and characteristics, which determine the resilience of the social-ecological system in consideration.

\subsection{Social dilemmas in common-pool resources and public goods}

A social dilemma is a situation in which pursuing own interests is detrimental to the collective interests. Social dilemmas are everywhere in daily life and are at the heart of many social, environmental, and economic problems. In the context of local resource governance, users are privately better off when overharvesting the resource than restraining from harvesting following socially-binding agreements or norms, but doing so may lead to depletion of the resource. In a modelling setting, social dilemma is typically defined following the tradition of game theory. Dawes (1980) defines the social dilemma following the prisoner's dilemma game as a situation in which defecting yields better personal outcomes than cooperating regardless of what others do, but mutual cooperation yields better social outcomes than mutual defection. In real world problems, however, social dilemmas are ingrained in much more diverse situations depending 
on the benefit structure of mutual cooperation and mutual defection (Kollock 1998). This is especially true when it comes to social dilemmas faced by multiple persons who have repeated interactions (Kollock 1998) and when the payoff is uncertain (Dawes 1980). For a more comprehensive review on social dilemmas and discussions with regard to the context of a common-pool resource, see e.g., Van Lange et al. (2013) and Jager (2000).

In the context of natural resource management, the social dilemmas are either concerned with common pool resources or with public goods. In this thesis, Chapter 2 analyses the role of a social dilemma arising from the sharing of water as a common-pool resource, using an agentbased modelling framework. While the social dilemma in a common-pool resource is typically sketched as the tension between short-term and long-term interests, here it considers a social dilemma arising from seasonal variations of resource availability. This case is very prevalent in the context of small-scale irrigation systems in tropical regions where on may have more than one harvesting season (Pérez et al. 2016).

Most of the studies in the commons literature, mainly focus on conditions under which users can overcome social dilemmas related to resource extraction, whereas problems related to provision of the resource are largely overlooked (Anderies et al. 2004). In many real-world situations, however, both problems are strongly coupled and manifest at multiple scales (Gardner et al. 1990; Ostrom 2010; 2009). Chapter 3 \& 4 thus analyze social dilemmas considering the combined problem of resource extraction and provision of water infrastructure as a public good. While a few studies also considered coupled social dilemmas (Botelho et al. 2015; Solstad and Brekke 2011), findings from those studies rely on the assumption that benefits from contributing to a public good is linearly dependent on users' contribution level. In practice, however, benefits can be nonlinear and exhibit thresholds. The dynamics of restraining resource use and contributing to public goods becomes even more interesting when also exogenous changes occur. For example, climate change may affect resource availability or actions by other communities or altered regulations by governmental agencies tend to affect scarcity and ultimately also resources users' harvesting behavior.

The institutional setting plays an important role in mediating individual and group interests to mitigate risks and impacts of climate change (Agrawal 2008). A key requirement for successful resource governance - be it formal or informal - is compliance with rules, regulations or norms (Bastakoti and Shivakoti 2012; Shreedhar et al. 2019; Tavoni et al. 2012). Without effective enforcement of rules and norms, a self-interested individual has no incentive to follow the 
prescribed rules and norms because doing so is costly to the individual. A voluntary mechanism to sustain cooperation thus may be at risk due to free riding. This raises the obvious question under which conditions users choose to implement appropriate institutional arrangements to safeguard cooperation and how it relates to the cost of institutions (Dannenberg and Gallier 2019). Chapter 5 focuses on a public good dilemma, which is studied in a field experimental setting. As cooperative arrangement enforced through peer enforcement may be fragile under climate change, it is necessary to consider alternative governance arrangement through formal institutions. In small-scale irrigation systems, one can often observe institutions that are formally organized, but emerged decentrally through, for example, farmer organizations. Such institution self-organizes resource sharing as well as ensuring the proper maintenance of the shared infrastructure. Further, it is sometimes unclear how this form of institution is explicitly different from that of the informal institutions such as social norms, since the rules and regulations of the farmer organizations are typically incorporating local practices, norms, and laws. While differentiating both institutions is not the main focus of this thesis, understanding to what extent institutions are supported is crucially important, especially considering the individual experience of resources with existing institution.

\subsection{Resource scarcity and cooperation}

In the commons literature, findings on the interaction between resource scarcity and cooperation are inconclusive. On the one hand, the literature suggests that resource scarcity enhances cooperation (Osés-Eraso and Viladrich-Grau 2007; Osés-Eraso et al. 2008; Schlüter et al. 2016; Nie et al. 2020). This line of literature provides various explanations for why concerns about resource scarcity may foster cooperation (Osés-Eraso and Viladrich-Grau 2007), looking at the sources of scarcity (either environmental or human-induced) (Osés-Eraso et al. 2008), costs and benefits of cooperation in relation to punishment strengths (Schlüter et al. 2016), and concerns about the future decline of the resource (Finkbeiner et al. 2018). On the other hand, scarcity may also undermine cooperation (Grossman and Mendoza 2003; Blanco et al. 2015; Richter et al. 2013; Pfaff et al. 2015; Gatiso et al. 2015). Various explanations for the decrease in cooperation include increasing competition for the resource (Grossman and Mendoza 2003; Blanco et al. 2015), and opportunistic extraction of the resource by selfish individuals (Richter et al. 2013). 
While scarcity could have significant implication for the emergence of collective action, other contextual factors such as inequality and heterogeneity may have a role in promoting or undermining cooperation (Bisaro and Hinkel 2016; Bardhan et al. 2007). In the context of local water governance, land inequality and heterogeneity among users may pose an obstacle towards cooperation (Marchiori 2014; Kun and Dieckmann 2013). This thesis hypothesizes that scarcity may interact with other contextual factors such as user attributes and institutions. Understanding the effects of those mediating factors on cooperation thus can provide more insights into the self-governance of the social-ecological system. In particular, Chapter 2 looks at the interactions between resource scarcity and agent's inequality and heterogeneity and its effect on the emergence and evolution of social norms of water sharing among farmers. Chapter 3 investigates whether the institution in place may interact with scarcity and thus affect cooperation. Chapter 5 analyses to what extent individual experiences about resource scarcity in the past may explain the support of costly institutions that ensure high cooperation or more effective enforcement.

\subsection{Social norms, institutions, and water governance}

Institutions can be broadly defined as rules that guide or constrain behavior of agents and their interactions (North 1991). Institutions consist of rules and norms which can be informally or informally arranged. Both forms of institutions are different in several aspects, but most importantly in terms of enforcement mechanisms (Yeboah-Assiamah et al. 2017). In the context of natural resource governance, enforcement is a key for all governance arrangements. Without effective enforcement of rules and norms, a self-interested individual is tempted to freeride on effort of others, since the benefit are personal, whereas the costs are shared by all resource beneficiaries.

Informal institutions such as social norms play an important role in water governance, especially under climate change (Pahl-Wostl 2009; Herrfahrdt-Pähle and Pahl-Wostl 2012). A social norm is a common behavioral pattern that is socially approved, collectively understood and maintained through social interactions within a group (Nyborg et al. 2016). In small communities that self-govern common pool resources, social norms can play important roles to facilitate cooperation (Ostrom 2000). With appropriate conditions, social norms can even address global problems (Nyborg et al. 2016). A social norm, however, is not always obeyed, especially when following it does not yield better personal outcomes. An enforcement 
mechanism is thus needed to sustain social norms. Regarding self-governance of a local resource, social norms are typically enforced through peer punishments (Fehr and Gächter 2002; Gächter et al. 2008; Ostrom 2000). Sometimes, especially in large groups where interactions are infrequent, social norms can be enforced through interpersonal communications, e.g. due to reputational concerns among their members (Fehr 2004) or moral motivations (Brekke et al. 2003; Efferson and Fehr 2018). For a review on the foundation of human cooperation in relation to social norms, see e.g., Fehr and Schurtenberger (2018).

In other cases, water is managed through a formal institution in which written rules or regulations are centrally enforced by a management agency or a third party. Various experimental and theoretical studies examine the performance of peer enforcement and central enforcement mechanisms to regulate common pool resource use (Fehr and Williams 2018; Zhang et al. 2014; Sigmund et al. 2010). Using an economic experiment, Traulsen et al. (2012) find that central enforcement is preferred over peer enforcement, especially when social sanctions can also be targeted at those who are unwilling to correct and enforce behavior of peers. With a similar theoretical model, Sigmund et al. (2010) have shown that peer sanctioning may be more effective and more efficient than central enforcement but only if it is not possible to commit everyone to contribute to a central sanctioning mechanism. An entirely open question concerns to what extent resource users may support such institutions if they are about to be implemented or already in place. Few studies have looked at how climate change may affect cooperative arrangements, mainly on how resource users adjust their harvesting level in response to increasing levels of resource scarcity. However, how peer and central enforcement mechanisms respond to and perform under scarcity conditions remains less understood, and is the focus of this thesis.

\subsection{Evolutionary agent-based modelling and collective action}

Agent-based modelling (ABM) has been extensively used in various disciplines, including agricultural, environmental, and ecological economics (Berger 2001; Rasch et al. 2016), and for analysis of social-ecological system dynamics (Schlüter et al. 2016). A social-ecological system (SES) is characterized by nonlinear dynamics and thresholds, heterogeneity, and emergence properties (Liu et al. 2007; Hull et al. 2015). In the analysis of a SES and its resilience, $\mathrm{ABM}$ is widely used for its flexibility to incorporate heterogeneity of agents, dynamic feedbacks of a complex adaptive system, and emergence of collective action from 
micro-level interactions (Janssen and Ostrom 2007; Schlüter et al. 2019). For a review on the use of an ABM for analysis of SES, see e.g., Schulze et al. (2017).

For the study of collective action and the evolution of cooperation, ABM is a promising tool since it allows modelers to specify behavior of agents and observe the interactions over time. Since human behavior is complex and adaptive, incorporating it into a formal model thus remains a challenge (Schlüter et al. 2017). In real world situation, human decisions are typically based on incomplete information, which can evolve based on adaptation and learning. The agent-based modelling approach is thus a promising tool to study human decision making in such an adaptive, evolving and complex environment, which is the key feature in the analysis of the evolution of cooperation (Heckbert et al. 2010; Janssen and Ostrom 2007). For a comprehensive discussions on agent-based modelling for the study of collective action, see e.g., Poteete et al. (2010).

This thesis employs agent-based modelling in Chapter 2, Chapter 3, and Chapter 4 since agents are adaptive and heterogeneous in terms of their skills and endowments, as well as resource extraction and investment levels. Further, this modelling approach also allows for the analysis of diverse coupling effects between scarcity and contextual variables on cooperation. For example, I investigate how social learning arises from different modes of social interactions, e.g. between a focal agent and his immediate neighbours and with partners from a different community. Finally, this thesis (Chapter 4) also uses agent-based simulation to study multilevel interactions within and between communities of resource users who jointly harvest water as a common-pool resource as well as invest to a public good such as an irrigation system. In such a complex system with multilevel interactions, other studies also employ this modelling approach, e.g. the study of a cross-scale resilience of a commons rangeland system in South Africa (Rasch et al. 2017), and the evolution of cooperative arrangement of multiple communities in Bali (Lansing et al. 2017). 


\subsection{Overview of this thesis}

\subsubsection{Thesis objectives}

The main focus of this thesis is to understand how institutional arrangements interact with contextual factors in the field to affect the resilience of cooperative resource management under climate change. In particular, I focus on informal institutions such social norms of cooperation, in which the norm is enforced by costly peer punishment (Chapter 2) or through reputational concerns (Chapter $3 \& 4$ ), as well as on formal institutions, in which the preference over two enforcement mechanisms, namely a costless weak enforcement versus a costly strict enforcement is studied (Chapter 5). Firstly, in Chapter 2, 3, \& 4 I focus on how the interactions between resource scarcity and user attributes as well as institutions affect cooperation. Specifically, Chapter 2 analyses how resource scarcity interacts with inequality and affects the emergence and stability of cooperative arrangements. While Chapter 2 considers the effect of scarcity that is exogenously driven e.g. by climate change, Chapter $3 \& 4$ considers the case in which scarcity is endogenous through the presence of a public good. Chapter 4 furthermore investigates how the effect of scarcity spreads between two communities who are linked through the presence of a public good. Findings from Chapter $2 \& 3$ highlight the role of informal and formal institutions, in particular enforcement mechanisms and existing institutional settings, in moderating or intensifying scarcity effects and thus affecting cooperation. Chapter 5 tests this in the field through lab-in-the field experiments to see if (i) a strict, but costly enforcement mechanism and (ii) costly formal institution, will be selected by users, and further investigate how this depends on contextual factors, such as individual experience of resource users with regard to scarcity. Overall, this thesis aims to answer the following questions.

1. How does resource scarcity and inequality among agents jointly affect the emergence and resilience of social norms? (Chapter 2)

2. How does the need to contribute to water infrastructure affect the cooperation to restrain water use and which mechanisms explain the emergence of institutional traps and prosperity? (Chapter 3)

3. Under which conditions does cooperation emerge and spread between communities and how does this depend on the level of interaction? (Chapter 4) 
4. Under which conditions would users choose to implement appropriate institutional arrangements to safeguard cooperation and how does it relate to the cost of institutions? (Chapter 5)

\subsubsection{Thesis outline}

This thesis focuses on the self-governance of local water resources under climate change. In particular, I analyze under which conditions and mechanisms of cooperative arrangements can be established and maintained, through the lens of institutions.

In Chapter 2 I developed an evolutionary agent-based model to study the evolution of water sharing norms among farmers who harvest water as a common-pool resource over two harvesting seasons. The norm prescribes to save water in the rainy season for use in the dry season. Agents face the social dilemma of using the water immediately for personal benefit or save it for later use for collective benefit. The norm is enforced through a costly peer enforcement mechanism. This chapter adds to the literature of the commons by focusing on the social dilemma arising from seasonal resource allocation. Further, the chapter analyses the role of scarcity and contextual factors, in fostering or eroding cooperation.

In Chapter 3 I developed an agent-based model in which users have to decide how much to contribute to common water infrastructure and how much water to extract. The starting point were stylized facts obtained from field experiments to show that a larger share of conditional cooperators in villages correlates positively with infrastructure quality and negatively with experienced scarcity. The model formalized the mechanism of conditional cooperation, where decisions are based on economic considerations, but also reputational concerns, where the own decision is evaluated against the social norm in the community.

In Chapter 4, I developed an agent-based model to analyse under what conditions cooperation emerges and spread across two communities who share water as a common-pool resource and infrastructure as a public good. Agents make decisions on levels of extraction and investment over time, starting from heterogeneous strategies. While taking more resource or investing less to its production makes an agent economically better off in the short-term, doing so erodes his self-image which can potentially inflict loss on his economic performance in the long-term. Agents make decisions balancing between doing well economically and doing good morally by learning from the best performing neighbours and other agents in a different community. 
Chapter 5 investigates the role of individual experience of scarcity on the support of institutions. In a field experiment with farmers in Campong Chhnang (Cambodia) individuals played oneshot public good games in groups of three, with an option to vote for a preferred institution. In the first setting, the choice is between a costless voluntary system and a costly tax system that requires a minimum contribution to the public good. In the second setting, the choice is between a costless weak enforcement mechanism in which rule breakers might not be detected and punished, and a costly strict enforcement mechanism in which every rule breaker is detected and punished. In both cases, contributions are made after having voted, but before knowing what others would have voted to obtain a clean measure for behavior in both institutional settings. 


\section{References}

Agrawal, Arun. 2008. "The Role of Local Institutions in Adaptation to Climate Change.” Anderies, John M, Marco a Janssen, and Elinor Ostrom. 2004. "A Framework to Analyze the Robustness of Social-Ecological Systems from an Institutional Perspective.” Ecology and Society 9 (1): 1-18. https://doi.org/18.

Bardhan, Pranab, Maitreesh Ghatak, and Alexander Karaivanov. 2007. "Wealth Equality and Collective Action." Journal of Public Economics 91 (9): 1843-74. https://doi.org/10.1016/j.jpubeco.2007.03.002.

Bastakoti, Ram C., and Ganesh P. Shivakoti. 2012. "Rules and Collective Action: An Institutional Analysis of the Performance of Irrigation Systems in Nepal.” Journal of Institutional Economics 8 (2): 225-46. https://doi.org/10.1017/S1744137411000452.

Berger, Thomas. 2001. “Agent-Based Spatial Models Applied to Agriculture: A Simulation Tool for Technology Diffusion, Resource Use Changes and Policy Analysis." Agricultural Economics 25 (2-3): 245-60.

Bisaro, Alexander, and Jochen Hinkel. 2016. "Governance of Social Dilemmas in Climate Change Adaptation." Nature Climate Change 6 (4): 354-59.

Blanco, Esther, Maria Claudia Lopez, and Sergio Villamayor-Tomas. 2015. "Exogenous Degradation in the Commons: Field Experimental Evidence.” Ecological Economics 120: 430-39. https://doi.org/10.1016/j.ecolecon.2015.03.028.

Botelho, Anabela, Ariel Dinar, Lígia M.Costa Pinto, and Amnon Rapoport. 2015. "Promoting Cooperation in Resource Dilemmas: Theoretical Predictions and Experimental Evidence." Journal of Behavioral and Experimental Economics 54: 40-49. https://doi.org/10.1016/j.socec.2014.11.007.

Brekke, Kjell Arne, Snorre Kverndokk, and Karine Nyborg. 2003. “An Economic Model of Moral Motivation.” Journal of Public Economics 87 (9-10): 1967-83. https://doi.org/10.1016/S0047-2727(01)00222-5.

Dannenberg, Astrid, and Carlo Gallier. 2019. "The Choice of Institutions to Solve Cooperation Problems: A Survey of Experimental Research.” Experimental Economics. https://doi.org/10.1007/s10683-019-09629-8.

Dawes, Robyn M. 1980. “Social Dilemmas.” Annual Review of Psychology 31 (1): 169-93.

Efferson, Charles, and Ernst Fehr. 2018. "Simple Moral Code Supports Cooperation.” Nature 555 (7695): 169-70. https://doi.org/10.1038/d41586-018-02621-X.

Elliott, Joshua, Delphine Deryng, Christoph Müller, Katja Frieler, Markus Konzmann, Dieter 
Gerten, Michael Glotter, et al. 2014. "Constraints and Potentials of Future Irrigation Water Availability on Agricultural Production under Climate Change." Proceedings of the National Academy of Sciences 111 (9): 3239-44.

Fehr, Ernst. 2004. “Don’t Lose Your Reputation.” Nature 432 (November): 2-3. https://doi.org/10.1038/432449a.

Fehr, Ernst, and Simon Gächter. 2002. “Altruistic Punishment in Humans.” Nature 415 (6868): 137-40. https://doi.org/10.1038/415137a.

Fehr, Ernst, and Ivo Schurtenberger. 2018. "Normative Foundations of Human Cooperation Review-Article." Nature Human Behavior 2 (7): 458-68. https://doi.org/10.1038/s41562-018-0385-5.

Fehr, Ernst, and Tony Williams. 2018. "Social Norms, Endogenous Sorting and the Culture of Cooperation." 7003.

Finkbeiner, E. M., F. Micheli, A. Saenz-Arroyo, L. Vazquez-Vera, C. A. Perafan, and J. C. Cárdenas. 2018. "Local Response to Global Uncertainty: Insights from Experimental Economics in Small-Scale Fisheries." Global Environmental Change 48 (October 2017): 151-57. https://doi.org/10.1016/j.gloenvcha.2017.11.010.

Gächter, Simon, Elke Renner, and Martin Sefton. 2008. "The Long-Run Benefits of Punishment.” Science 322 (5907): 1510. https://doi.org/10.1126/science.1164744.

Gardner, Roy, Elinor Ostrom, and James M. Walker. 1990. “The Nature of Common-Pool Resource Problems." Rationality and Society 2 (3): 335-58.

Gatiso, Tsegaye T, Björn Vollan, and Ernst-august Nuppenau. 2015. "Resource Scarcity and Democratic Elections in Commons Dilemmas : An Experiment on Forest Use in Ethiopia." Ecological Economics 114: 199-207. https://doi.org/10.1016/j.ecolecon.2015.04.005.

Grossman, Herschel I., and Juan Mendoza. 2003. "Scarcity and Appropriative Competition." European Journal of Political Economy 19 (4): 747-58.

Haddeland, Ingjerd, Jens Heinke, Hester Biemans, Stephanie Eisner, Martina Flörke, Naota Hanasaki, Markus Konzmann, et al. 2014. “Global Water Resources Affected by Human Interventions and Climate Change." Proceedings of the National Academy of Sciences 111 (9): 3251-56.

Heckbert, Scott, Tim Baynes, and Andrew Reeson. 2010. “Agent-Based Modeling in Ecological Economics.” Annals of the New York Academy of Sciences 1185 (1): 39-53. Herrfahrdt-Pähle, Elke, and Claudia Pahl-Wostl. 2012. "Continuity and Change in SocialEcological Systems: The Role of Institutional Resilience." Ecology and Society 17 (2). 
https://doi.org/10.5751/ES-04565-170208.

Hull, V, MN Tuanmu, Jianguo Liu, and T Lags. 2015. "Synthesis of Human-Nature Feedbacks." Ecology and Society 20 (3): 17.

http://www.ecologyandsociety.org/vol20/iss3/art17/ES-2015-7404.pdf.

Jaeger, William K., Adell Amos, Daniel P. Bigelow, Heejun Chang, David R. Conklin, Roy Haggerty, Christian Langpap, et al. 2017. "Finding Water Scarcity amid Abundance Using Human-Natural System Models." Proceedings of the National Academy of Sciences 114 (45): 11884-89.

Jager, Wander. 2000. “The Commons Dilemma.” In Modelling Consumer Behavior, 9-20. Janssen, Marco A, and Elinor Ostrom. 2007. “Adoption of a New Regulation for the Governance of Common- Cool Resources by a Heterogeneous Population.” In Inequality, Cooperation, and Environmental Sustainability, edited by Jean-Marie Baland, Pranab K. Bardhan, and Samuel Bowles, 60-96. Princeton University Press. Kollock, Peter. 1998. "Social Dilemmas: The Anatomy of Cooperation." Annual Review of Sociology 24: 182-214. https://doi.org/10.1146/annurev.soc.24.1.183.

Kun, Adam, and Ulf Dieckmann. 2013. "Resource Heterogeneity Can Facilitate Cooperation." Nature Communications 4: 2453.

Lange, Paul A M Van, Jeff Joireman, Craig D. Parks, and Eric Van Dijk. 2013. “The Psychology of Social Dilemmas: A Review." Organizational Behavior and Human Decision Processes 120 (2): 125-41. https://doi.org/10.1016/j.obhdp.2012.11.003. Lansing, J. Stephen, Stefan Thurner, Ning Ning Chung, Aurelie Coudurier-curveur, Cagil Karakas, Kurt A. Fesenmyer, and Lock Yue Chew. 2017. “Adaptive Self-Organization of Bali's Ancient Rice Terraces." Proceedings of the National Academy of Sciences 114 (25): 6504-6509. https://doi.org/10.1073/pnas.1605369114.

Liu, Jianguo, Thomas Dietz, S. Carpenter, Marina Alberti, Carl Folke, Emilio Moran, Alice N Pell, et al. 2007. "Complexity of Coupled Human and Natural Systems.” Science 317 (5844): 1513-16.

Marchiori, Carmen. 2014. "Inequality and Rules in the Governance of Water Resources." Ecological Economics 105: 124-29.

Muneepeerakul, Rachata, and John M. Anderies. 2020. "The Emergence and Resilience of Self-Organized Governance in Coupled Infrastructure Systems." Proceedings of the National Academy of Sciences of the United States of America 117 (9): 4617-22. https://doi.org/10.1073/pnas.1916169117.

Nie, Zihan, Xiaojun Yang, and Qin Tu. 2020. "Resource Scarcity and Cooperation : Evidence 
from a Gravity Irrigation System in China.” World Development 135: 105035.

https://doi.org/10.1016/j.worlddev.2020.105035.

North, Douglass C. 1991. "Institutions.” Journal of Economic Perspectives 5 (1): 97-112.

Nyborg, By Karine, John M Anderies, Astrid Dannenberg, Therese Lindahl, Caroline Schill, Maja Schlüter, W.N. Adger, et al. 2016. "Social Norms as Solutions.” Science 354 (6308): 42-43.

Osés-Eraso, Nuria, Frederic Udina, and Montserrat Viladrich-Grau. 2008. "Environmental versus Human-Induced Scarcity in the Commons: Do They Trigger the Same Response?" Environmental and Resource Economics 40 (4): 529-50. https://doi.org/10.1007/s10640-007-9168-6.

Osés-Eraso, Nuria, and Montserrat Viladrich-Grau. 2007. “Appropriation and Concern for Resource Scarcity in the Commons: An Experimental Study.” Ecological Economics 63 (2-3): 435-45. https://doi.org/10.1016/j.ecolecon.2006.11.016.

Ostrom, Elinor. 2000. “Collective Action and the Evolution of Social Norms." Journal of Economic Perspectives 14 (3): 137-58.

- 2009. "A General Framework for Analyzing Sustainability of Social-Ecological Systems.” Science 325 (5939): 419-22.

_.2010. "Beyond Markets and States : Polycentric Governance of Complex Economic Systems.” American Economic Review 100 (3): 641-72.

Pahl-Wostl, Claudia. 2009. “A Conceptual Framework for Analysing Adaptive Capacity and

Multi-Level Learning Processes in Resource Governance Regimes." Global

Environmental Change 19 (3): 354-65. https://doi.org/10.1016/j.gloenvcha.2009.06.001. Perez, I, M A Janssen, and J M Anderies. 2016. "Food Security in the Face of Climate Change: Adaptive Capacity of Small-Scale Social-Ecological Systems to Environmental Variability." Global Environmental Change 40: 82-91.

https://doi.org/10.1016/j.gloenvcha.2016.07.005.

Pérez, Irene, Marco A. Janssen, and John M. Anderies. 2016. "Food Security in the Face of Climate Change: Adaptive Capacity of Small-Scale Social-Ecological Systems to Environmental Variability." Global Environmental Change 40: 82-91. https://doi.org/10.1016/j.gloenvcha.2016.07.005.

Pfaff, Alexander, Maria Alejandra Vélez, Pablo Andres Ramos, and Adriana Molina. 2015.

"Framed Field Experiment on Resource Scarcity \& Extraction: Path-Dependent

Generosity within Sequential Water Appropriation.” Ecological Economics 120: 416-29. Poteete, Amy R., Marco A. Janssen, and Ostrom Elinor. 2010. “Agent-Based Models of 
Collective Action." In Working Together: Collective Action, the Commons, and Multiple Methods in Practice. Princeton University Press.

Rasch, Sebastian, Thomas Heckelei, Roelof Oomen, and Christiane Naumann. 2016.

"Cooperation and Collapse in a Communal Livestock Production SES Model-a Case from South Africa." Environmental Modelling and Software 75: 402-13.

Rasch, Sebastian, Thomas Heckelei, Hugo Storm, Roelof Oomen, and Christiane Naumann. 2017. "Multi-Scale Resilience of a Communal Rangeland System in South Africa." Ecological Economics 131: 129-38. https://doi.org/10.1016/j.ecolecon.2016.08.012.

Richter, A., D. van Soest, and J. Grasman. 2013. "Contagious Cooperation, Temptation, and Ecosystem Collapse.” Journal of Environmental Economics and Management 66 (1): $141-58$.

Schewe, Jacob, Jens Heinke, Dieter Gerten, Ingjerd Haddeland, Nigel W. Arnell, Douglas B. Clark, Rutger Dankers, et al. 2014. "Multimodel Assessment of Water Scarcity under Climate Change." Proceedings of the National Academy of Sciences 111 (9): 3245-50.

Schill, Caroline, John M. Anderies, Therese Lindahl, Carl Folke, Stephen Polasky, Juan Camilo Cárdenas, Anne Sophie Crépin, Marco A. Janssen, Jon Norberg, and Maja Schlüter. 2019. "A More Dynamic Understanding of Human Behavior for the Anthropocene.” Nature Sustainability 2 (12): 1075-82. https://doi.org/10.1038/s41893019-0419-7.

Schlüter, Maja, Andres Baeza, Gunnar Dressler, Karin Frank, Groeneveld Jürgen, Jager Wander, Marco A. Janssen, et al. 2017. “A Framework for Mapping and Comparing Behavioral Theories in Models of Social-Ecological Systems." Ecological Economics 131: 21-35. https://doi.org/10.1016/j.ecolecon.2016.08.008.

Schlüter, Maja, Birgit Müller, and Karin Frank. 2019. "The Potential of Models and Modeling for Social-Ecological Systems Research: The Reference Frame ModSES.” Ecology and Society 24 (1). https://doi.org/10.5751/ES-10716-240131.

Schlüter, Maja, Alessandro Tavoni, and Simon Levin. 2016. "Robustness of Norm-Driven Cooperation in the Commons." Proc. R. Soc. B 283 (1822).

Schulze, Jule, Birgit Müller, Jürgen Groeneveld, and Volker Grimm. 2017. “Agent-Based Modelling of Social-Ecological Systems: Achievements, Challenges, and a Way Forward." Journal of Artificial Societies and Social Simulation 20 (2): 8. https://doi.org/10.18564/jasss.3423.

Shreedhar, Ganga, Alessandro Tavoni, and Carmen Marchiori. 2019. "Monitoring and Punishment Networks in a Common-Pool Resource Dilemma." Environment and 
Development Economics, 1-29. https://doi.org/10.1017/S1355770X19000457.

Sigmund, Karl, Hannelore De Silva, Arne Traulsen, and Christoph Hauert. 2010. "Social Learning Promotes Institutions for Governing the Commons." Nature 466 (7308): 86163. https://doi.org/10.1038/nature09203.

Solstad, Jan Tore, and Kjell Arne Brekke. 2011. "Does the Existence of a Public Good Enhance Cooperation among Users of Common-Pool Resources?" Land Economics 87 (2): 335-45. https://doi.org/10.1353/lde.2011.0023.

Tavoni, Alessandro, Maja Schlüter, and Simon Levin. 2012. "The Survival of the Conformist: Social Pressure and Renewable Resource Management." Journal of Theoretical Biology 299: 152-61. https://doi.org/10.1016/j.jtbi.2011.07.003.

Traulsen, A., T. Rohl, and M. Milinski. 2012. “An Economic Experiment Reveals That Humans Prefer Pool Punishment to Maintain the Commons." Proceedings of the Royal Society B: Biological Sciences 279 (1743): 1471-2954.

Veldkamp, T.I.E., Y. Wada, J.C.J.H. Aerts, P. Döll, S. N. Gosling, J. Liu, Y. Masaki, et al. 2017. "Water Scarcity Hotspots Travel Downstream Due to Human Interventions in the 20th and 21st Century." Nature Communications 8. https://doi.org/10.1038/ncomms15697.

Wada, Yoshihide, Marc F P Bierkens, Ad De Roo, Paul A Dirmeyer, James S Famiglietti, Naota Hanasaki, Megan Konar, Junguo Liu, and Hannes Müller Schmied. 2017. "Human-Water Interface in Hydrological Modelling: Current Status and Future Directions." Earth Syst. Sci 215194: 4169-93. https://doi.org/10.5194/hess-21-41692017.

Yeboah-Assiamah, Emmanuel, Kobus Muller, and Kwame Ameyaw Domfeh. 2017. "Institutional Assessment in Natural Resource Governance: A Conceptual Overview." Forest Policy and Economics 74: 1-12. https://doi.org/10.1016/j.forpol.2016.10.006. Yu, David J, Murad R Qubbaj, Rachata Muneepeerakul, John M Anderies, and Rimjhim M Aggarwal. 2015. "Effect of Infrastructure Design on Commons Dilemmas in SocialEcological System Dynamics." Proceedings of the National Academy of Sciences of the United States of America 112 (43): 1410688112-. https://doi.org/10.1073/pnas.1410688112.

Zhang, Boyu, Cong Li, Hannelore De Silva, Peter Bednarik, and Karl Sigmund. 2014. "The Evolution of Sanctioning Institutions: An Experimental Approach to the Social Contract." Experimental Economics 17 (2): 285-303. https://doi.org/10.1007/s10683013-9367-7. 



\title{
Chapter 2-The resilience of social norms of cooperation under resource scarcity and inequality - an agent-based model on sharing water over two harvesting seasons
}

\author{
Tum Nhim ${ }^{1}$, Andries Richter ${ }^{1}$, Xueqin Zhu ${ }^{1}$ \\ ${ }^{1}$ Environmental Economics and Natural Resources Group, Wageningen University, P.O. Box \\ 8130, 6700 EW Wageningen, The Netherlands \\ Published as: \\ Nhim, T., Richter, A., \& Zhu, X. (2019). The resilience of social norms of cooperation under \\ resource scarcity and inequality_-An agent-based model on sharing water over two \\ harvesting seasons. Ecological Complexity, 40, 100709.
}

\begin{abstract}
Water governance remains a challenge for human societies, especially when the variation in resource inflow is large and the resource users are heterogeneous. We analyze with a coupled social-ecological systems (SES) model how socioeconomic and environmental changes affect the resilience of social norms governing resource use. In our model, agents have access to water as a common-pool resource and allocate it between rainy and dry seasons. While it is socially optimal to save water for the dry season, it is individually optimal to take water immediately. In our model, punishment of norm violators is the mechanism that may sustain cooperation. We show that the resilience of social norms could be affected by changes in socioeconomic and environmental conditions. Particularly, we find that social norms may collapse in times of resource scarcity and variability, especially if several drivers act in concert. Finally, we find that user heterogeneity in the form of different skills and inequality in land endowments may undermine cooperation. This implies that climatic changes and increased inequality - both potential drivers in the field - may affect community resilience and may lead to an erosion of social norms.
\end{abstract}

Keywords: agent-based modeling, cooperation, resource scarcity, inequality, resilience, social-ecological systems 


\subsection{Introduction}

Sharing a common-pool resource (CPR), such as water, remains a challenge for human societies. Wasteful overuse of such a resource typically arises from a social dilemma, which is defined as the conflict between individual and group interests. This form of collective action problem is especially pronounced in the case of a CPR, which is a rival resource (i.e. extraction by one user makes it unavailable to others) and non-exclusive (i.e. excluding others from appropriating such a resource is difficult or costly) (Gardner et al. 1990). In the absence of enforcement mechanisms, an individual has no incentive to restrain resource use, since benefits of taking the resource immediately are private, whereas the benefits of saving it for later use are shared by all resource users.

A CPR is usually part of an interconnected system of users, governing institutions and the biophysical system, which is often referred to as a social-ecological system (SES) (Ostrom 2009). A SES is generally understood as a complex adaptive system, in which micro-level interactions of agents lead to emergent properties at a macroscopic level that, in turn, affect actions and behavior of the agents (Levin et al. 2012). Such a system is characterized by complexities, namely nonlinear feedbacks, tipping points, heterogeneity of agents, and scaledependences, which may pose obstacles for successful governance of CPR (Liu et al. 2007; Levin et al. 2012).

A wealth of case studies have documented that local communities are able to sustain the commons by self-organizing and solving collective action problems (Ostrom 1990). Various key factors and mechanisms that may affect collective action have been identified, which include system productivity and scarcity, as well as the existence of norms or social capital (Ostrom 2009). However, how these factors and mechanisms link across scales to affect the long-term sustainability of resource use is not clearly understood (Ostrom 2009). Experimental work has shown that social norms - in the form of restraining individual resource use and

punishing noncooperative behavior - play crucial roles in sustaining resource use (Ostrom et al. 1992; Ostrom et al. 1994). Using evolutionary game theory, Sethi \& Somanathan (1996) have shown that punishments can enforce sustainable resource use, provided that defection is not very common initially. Their model features two alternative stable states - full cooperation and full defection - depending on initial conditions. The fragility of social norms has been further documented by Richter et al. (2013), who show that cooperation can suddenly collapse 
in response to exogenous drivers, such as technological progress or climatic changes. Nyborg et al. (2016) have suggested that tipping points may also play a role when social norms are established. For example, by making agents' behavior more observable (e.g. resource extraction) social sanctioning can create a tipping point from a vicious cycle of norm-violation to a virtuous cycle of norm-following behavior.

Despite empirical and theoretical evidence suggesting that cooperation can be maintained or suddenly collapse, an open question is how the resilience of social norms could be affected by socioeconomic and environmental changes. By altering biophysical conditions of resources, climate change can obscure the nature of social dilemmas in relation to resource sharing, which requires an understanding of contextual factors such as agents' heterogeneity to overcome social dilemmas (Bisaro \& Hinkel 2016). For example, climate change is expected to aggravate water scarcity (Schewe et al. 2014; Haddeland et al. 2014) and increase water variability in time and space (Jaeger et al. 2017). How severe the impact is, however, depends on the spatial location of the irrigation areas and the strategic decisions of neighboring farmers who share the water (Esteve et al. 2015).

The objective of this paper is to analyze how the emergence and resilience of social norms of cooperation depends on external disturbances, as well as heterogeneity and inequality of users. In particular, we develop an agent-based model (ABM) to investigate how resource scarcity and inequality among agents may jointly affect the resilience of social norms in a community that extracts water for irrigation from a joint river. Our contribution to the literature is twofold. First, we analyze the case where water is used in two seasons for irrigation purposes, which is relevant in many real world settings. This is especially true for irrigation systems in Asia, where water availability varies considerably between rainy and dry seasons due to the effect of monsoon precipitation (Schewe et al. 2014; Elliott et al. 2014). A social dilemma arises, because it is socially optimal to save water in the rainy season, while the benefits of doing so are shared among all farmers, including those who have not restrained their water use in the rainy season. We analyze to what extent social norms can mitigate such social dilemma. Second, we investigate how the resilience of social norms could be affected in times of resource scarcity and variability and by changes in socioeconomic and environmental conditions.

Previous theoretical studies have demonstrated the crucial roles of social norms and punishment in facilitating cooperation and the implications for the sustainable use of a shared resource in a small community (Sethi \& Somanathan 1996; Noailly et al. 2003). On the one hand, such 
studies aim at understanding the emergence and evolution of cooperative harvesting strategies (Sethi \& Somanathan 1996; Tavoni et al. 2012; Richter et al. 2013; Lewis \& Dumbrell 2013) including cases where cooperation and defection may co-exist. On the other hand, such studies contribute to understanding the extent to which such norm-guided cooperation is robust against socioeconomic and environmental changes (Schlüter et al. 2016; Richter \& Dakos 2015; Brandt \& Merico 2013).

Agent-based modelling (ABM) has been extensively used in various disciplines (Heath et al. 2009), including agricultural and ecological economics (Berger 2001; Rasch et al. 2016), and for analysis of SES dynamics and tipping points (An et al. 2014; Schlüter et al. 2016; Siekmann 2015). ABM is widely used for its flexibility to incorporate heterogeneity of agents (An 2012; Schlüter et al. 2012), and particularly suited to analyze the emergence of collective action from micro-level interactions (Bonabeau 2002). For instance, Janssen \& Ostrom (2007) use agentbased modeling to examine how heterogeneity among agents could contribute to the emergence and evolution of social norms, a feature which is difficult to include in a traditional game theoretical model that studies the evolution of cooperation. Similarly, Bausch (2014) employs agent-based simulations to test mechanisms that contribute to cooperation between groups, which is similar to the work of Gavrilets \& Richerson (2017), who focus more on competition between groups.

$\mathrm{ABM}$ is a useful tool to analyze resilience, which is understood as the capacity of the system to accommodate changes, while maintaining the system states within the equilibrium domains (Liu et al. 2007; An et al. 2014). For instance, Schlüter \& Pahl-Wostl (2007) use ABM to assess resilience of the SES under different water governance regimes to uncertainties of water availability in a river basin. In their study, resilience is evaluated as the capacity of the system to maintain both agricultural and fish production at or above an exogenously specified level, below which each production system collapses. Schlüter et al. (2016) analyze the resilience of social norms of cooperation to environmental changes such as changes in resource availability and variability. Similarly, Rasch et al. (2016) show that social norms emerge in times of ecological crises and enhance resilience of SES.

Our paper contributes to the question how resource scarcity affects cooperation in the commons, which remains poorly understood. One strand of literature argues that resource scarcity enhances cooperation. For example, using dynamic game theory, Osés-Eraso and ViladrichGrau (2007) show that concerns for resource scarcity can dampen resource extraction. Testing 
this in an experimental setting, Osés-Eraso et al. (2008) find that users tend to react to actual scarcity by reducing the appropriation level when resources become scarcer. In addition, it matters whether the sources of scarcity are environmental or human-induced (Osés-Eraso et al. 2008). Considering changes in availability and variability of a resource, Schlüter et al. (2016) have demonstrated that cooperation can collapse even in the case of resource abundance as long as norm-violators benefit in times of resource abundance. Increased scarcity, potentially mediated through resource variability, can enhance cooperation because scarcity favors cooperation in the sense that the benefits of violating the norms are smaller (less is to be extracted), while the sanctioning strength against norm-violators remains high when the resource becomes scarcer (Schlüter et al. 2016).

The other strand of literature posits that resource scarcity may give rise to collapse of cooperation among the users of the commons. For instance, resource scarcity may increase competition for resource appropriation which can lead to a faster rate of depletion (Grossman \& Mendoza 2003). This finding is in line with experimental evidence from Blanco et al. (2015), who have found that resource users increase their appropriation levels when the resource becomes scarcer, no matter whether the reduction in resource availability is abrupt or gradual. Users even tend to appropriate more resources if they experience scarcity in the past (Blanco et al. 2015). This is further supported by Pfaff et al. (2015), who find in another experiment that users tend to extract more if the resource is initially scarce, leading to erosion of collective action. In a similar vein, theoretical work has shown that cooperation may collapse in the wake of scarcity if scarcity increases the temptation to defect because cooperatively-minded individuals restrain themselves as an attempt to restore the resource (Richter et al. 2013).

Contextual factors such as inequality and user heterogeneity may interact with external disturbances and hence affect the evolution of social norms and self-organization (Bisaro \& Hinkel 2016). In the context of water governance at a local scale, unequal land endowments may have significant implication for the emergence of collective action. Land inequality may pose an obstacle towards cooperation, depending on the complementarity between land and water (Marchiori 2014). Kun and Dieckmann (2013) have also demonstrated that user heterogeneity has important implications for the emergence of cooperation. Inequality of an initial resource endowment can facilitate or hinder cooperation depending on the benefits of defection (Kun \& Dieckmann 2013). 
Clearly, both inequality among users and resource scarcity can impact the emergence and maintenance of cooperation in the commons. Previous papers have analyzed i) how social norms emerge and overcome social dilemmas in the case of equal access to the resource (Sethi \& Somanathan 1996; Tavoni et al. 2012; Richter \& Grasman 2013); ii) how availability and variability of the resource inflow affect cooperation among agents and the long-term use of a CPR (Schlüter et al. 2016); and iii) how resource inequality affects collective use of a resource (Marchiori 2014; Kun \& Dieckmann 2013; Rasch et al. 2016). However, how resource scarcity and inequality interact and hence affect cooperation in the use of common resources remains unexplored.

The organization of the paper is as follows. Section 2 is the presentation of the SES model, which consists of three components, namely the biophysical model, the economic model and the social dynamics model. Section 3 presents the results, investigating how resource scarcity and user heterogeneity affect cooperation. Section 4 concludes and discusses the findings.

\subsection{The model}

We consider a case of small-scale irrigation system in a community, which is part of a socialecological system (SES). The community consists of $N$ farmers having access to a commonpool resource - a joint river from which water is withdrawn for irrigating a single crop in two seasons of the year, namely rainy and dry seasons. In this irrigation system, a proportion of water can be saved in the rainy season for later use in the dry season to cope with seasonal variability.

When sharing a common-pool resource, farmers face a social dilemma in which individual and group interests are misaligned. While it would be socially optimal to save water in the rainy season for farming in the dry season, this is not individually rational, as the saved water may potentially be used by other farmers, making the individual who saved the water worse off. In the model, we allow social norms of cooperation to evolve that guide resource users' behavior regarding water use. Following the tradition of Sethi \& Somanathan (1996), we assume that some agents act cooperatively, while others act selfishly. The selfish agents (called defectors) are generally short-sighted and maximize their own short-term interests. Using the amount of water extracted as a yardstick to define behavioral choice, a defector thus extracts an amount of water greater than the social optimum. The cooperators follow social norms that guide water 
use in both seasons and punish defectors. If water is scarce, cooperators restrain water use in the rainy season and save for the dry season so that everyone is potentially better off.

Furthermore, the resilience of social norms of cooperation may be threatened by socioeconomic and environmental changes. When the sales price of the agricultural commodity increases in the rainy season, farmers may be tempted to use more water in the rainy season because the marginal profit from doing so is higher. Likewise, when water becomes scarcer, farmers who take water early on are the ones who benefit from farmers who are modest and try to save. Fig. 1 shows a conceptual model on how the dynamic interplay between water scarcity and variability in the natural system and inequality among agents in the socio-economic system may affect the behavior of farmers in water allocation.

To analyze inequality among agents in promoting or reducing cooperation, we distinguish two cases of our model: the basic case in which each agent is homogeneous in terms of farming skills and land endowments and the extended case in which agents are heterogeneous.

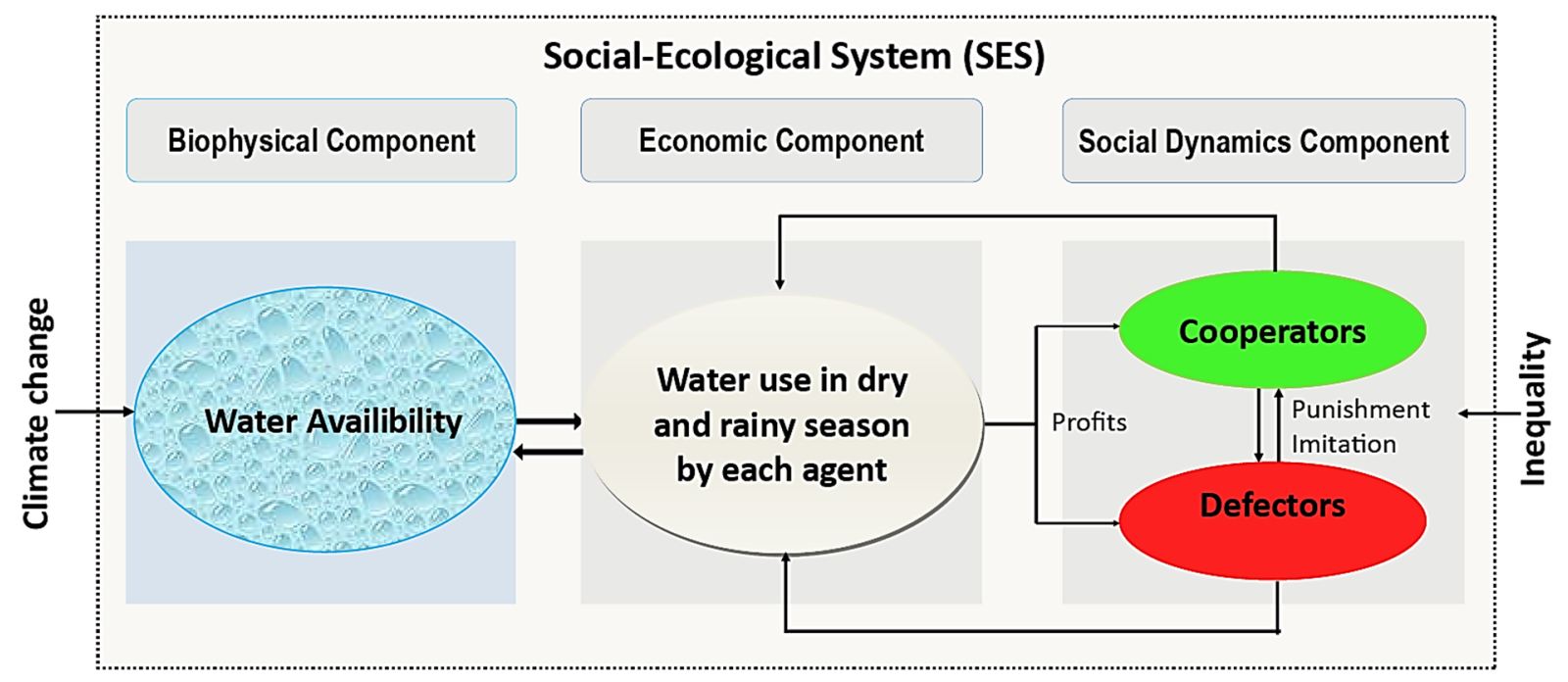

Fig.1. A conceptual model on the emergence of social norms in the wake of climate change and inequality. Climate change induces changes in rainfall pattern, which modifies the water inflow conditions in terms of quantity and seasonal variation. The changes in water availability thus affect the decisions of agents regarding seasonal water allocation following their norms that govern resource use, which then affect water availability. Differences in water use translate into differences in profits, giving rise to social dynamics. Cooperators punish defectors, while the more successful strategies are imitated. Inequality may act as a driver on the social dynamics, as individuals who are better off have a higher probability to be imitated. 


\subsubsection{Resource dynamics}

Water flows in a river are determined primarily by rainfall, which is stochastic. Water availability in the river thus fluctuates intra- and inter-annually. For simplicity, we define a random variable $Q_{r, t}$ as the total available water in the rainy season at year $t$ with a mean value of $\bar{Q}$ and a stochastic term $\varepsilon_{t}$, which denotes water variability. We assume that $\varepsilon_{t}$ is normally distributed, with zero mean and standard deviation $\sigma_{Q}$, i.e. $\varepsilon_{t} \sim N\left\{0, \sigma_{Q}{ }^{2}\right\}$. The quantity of water available in the rainy season $\left(Q_{r, t}\right)$ and dry season $\left(Q_{d, t}\right)$ is given by

$$
\begin{aligned}
Q_{r, t} & =\bar{Q}+\varepsilon_{t}, \\
Q_{d, t} & =Q_{r, t}-W_{r, t},
\end{aligned}
$$

where $W_{r, t}$ is the total amount of water withdrawn by all agents in the rainy season at year $t$. We assume that water can be saved in the rainy season for the dry season, and all water will be exhausted in the dry season. Water availability at a given year thus depends solely on water inflow occurring in the rainy season of that particular year. Hence, the social dilemma concerns inter-seasonal water allocations where agents make decision on water use in a given year. Water availability is influenced by external disturbances, e.g. climatic change, which induces shifts in rainfall patterns causing changes in both quantity and distribution of water inflow. We model the potential effects of climate change on water resource dynamics by varying the values of mean inflow $(\bar{Q})$ and standard deviation of water inflow $\left(\sigma_{Q}\right)$.

\subsubsection{Agent heterogeneity and inequality}

While the basic model comprising homogenous agents will be an important benchmark, we also consider the case where agents are heterogeneous. First, we introduce skill heterogeneity and take into account that not only water use determines yield, but also the skill of each agent, denoted by $\rho_{i}$. Skill $\rho_{i}$ is time-invariant and randomly distributed with mean $\bar{\rho}$ and standard deviation $\sigma_{\rho}$. Second, we consider land heterogeneity by assuming that each farmer is endowed with acreage $a_{i}$, which is agent-specific and randomly distributed with mean $\bar{a}$, and standard deviation $\sigma_{a}$. Furthermore, we introduce the presence of small and large landholders in the community by considering a bimodal distribution of land among the two groups. Specifically, it is modeled as the mixture of two Gaussian distributions, with two means and two variances, each representing the attributes of each farmer group with equal mixing proportions. The small landholders are endowed with a mean land size of $\bar{a}_{S}$ and the large landholders with $\bar{a}_{L}$. Both 
are assumed to have the same variance of land endowment and hence the same standard deviation $\left(\sigma_{a, S}=\sigma_{a, L}\right)$.

\subsubsection{Water allocations}

The economic model presents farmers' optimal land and water allocations for irrigating a single crop in the rainy (subscripted $r$ ) and dry (subscripted $d$ ) season at a given year. Each farmer (indexed by $i$ ) is endowed with land $a_{i}$, which can be cultivated twice a year: once in the rainy season denoted by $a_{i, r}$ and once in the dry season denoted by $a_{i, d}$. Yield per unit of land of agent $i$ is given by

$$
Y_{i} / a_{i}=\alpha+\beta w_{i}-\gamma w_{i}^{2},
$$

where $Y_{i}$ is the total production, $w_{i}$ is the amount of water use per unit land, and $\alpha, \beta$, and $\gamma$ are yield parameters. We assume that the price of the agricultural product is exogenous and the community can be considered a price-taker. However, the sales price in the rainy season $\left(P_{r}\right)$ is different from the price in the dry season $\left(P_{d}\right)$. We also assume a fixed farming cost $c$ for each unit of land cultivated, which may differ between seasons. Total profit of farmer $i$ in both seasons is given by

$$
\pi_{i}=\rho_{i} P_{r} Y_{i, r}\left(a_{i, r}, w_{i, r}\right)-c_{r} a_{i, r}+\rho_{i} P_{d} Y_{i, d}\left(a_{i, d}, w_{i, d}\right)-c_{d} a_{i, d} .
$$

Since profits depend linearly on land $a_{i}$ (see equations $3 \& 4$ ), the optimal use of land is prescribed by a "bang-bang" solution. If farming is profitable, $\rho_{i} P \partial Y_{i} / \partial a_{i} \geq c$, it is optimal to use all land in the relevant seasons. If it is profitable in both seasons, we have $a_{r}^{*}=a_{d}^{*}=a_{i}$. However, if farming is not economically viable, no land is used.

The decision on how much water to use is more complex. Defectors are short-sighted and withdraw water from the river system at the individual myopic optimum ignoring any benefits of saving water for the dry season. Cooperators use water at a socially optimal rate, typically saving water in the rainy season for the dry season. In particular, cooperators withdraw their "fair share" of water, i.e. the social optimal use of water divided by the number of farmers. When water becomes scarce, a social dilemma arises and short-sighted defectors are strictly better off than sustainably-minded cooperators. The main difference between cooperators and defectors is thus in how much water is used in the rainy season. In the dry season all water is used as long as marginal benefits are positive. For simplicity, we assume that the remaining water in the dry season is divided symmetrically among all agents, since the cooperative solution and the competitive solution (defined by the Cournot-Nash equilibrium) coincide in the dry season. 
Formally, short-sighted farmers simply maximize the profits from each farming season separately, subject to land constraints $a_{r, i} \leq a_{i}$ and $a_{d, i} \leq a_{i}$, which implies that using the same plot of land in both seasons (i.e. double cropping) is possible. The water constraint is the total water available in the system determined at the beginning of the year, while for the second season their water constraint corresponds to the amount of water left in the system. Hence, water constraints are given by $w_{r, i} \leq Q_{r} / a_{i}$ for the rainy season and $w_{d, i} \leq\left(Q_{r}-W_{r}\right) /\left(N a_{i}\right)$ for the dry season. In optimum, a short-sighted farmer equates marginal return from a unit of land with marginal cost of cultivating the land in each season, i.e. $\left(\rho_{i} P \partial Y_{i} / \partial a_{i}=c\right)$. For water allocation, water is used until marginal returns from using water is zero in each season, i.e. $\left(\rho_{i} P \partial Y_{i} / \partial w_{i}=0\right)$.

Cooperators allocate water for both seasons at the beginning of the year by maximizing total profit (see equation 4), subject to land constraints $a_{r, i} \leq a_{i}$ and $a_{d, i} \leq a_{i}$ and water constraints according to water availability in the system. In the rainy season, the water constraint for cooperators is the proportional share of the total water available in the system $\left(w_{r, i} \leq\right.$ $\left.Q_{r} /\left(N a_{i}\right)\right)$. In the dry season, their water constraint corresponds to the total water available left $\left(w_{d, i} \leq\left(Q_{r}-W_{r}\right) /\left(N a_{i}\right)\right)$. In optimum, a sustainably-minded farmer equates the marginal return to water in both seasons, i.e. $\rho_{i} P_{r} \partial Y_{i, r} / \partial w_{i, r}=\rho_{i} P_{d} \partial Y_{i, d} / \partial w_{i, d}$, and equates marginal returns to a unit of land used in each season with marginal costs of cultivated land, i.e. $\left(\rho_{i} P \partial Y_{i} / \partial a_{i}=c\right)$.

The optimization problem of cooperators and defectors can be solved analytically with inequality constraints, by forming the Lagrangian and using the Kuhn-Tucker conditions. It can also be solved numerically using the fmincon solver in MATLAB (Mathworks Inc.).

\subsubsection{Social dynamics}

After harvesting in both seasons has taken place, social dynamics unfold. The social dynamics build upon principles from evolutionary game theory (Sigmund \& Nowak 1999; Nowak 2006a) that depicts how cooperation and defection as strategies evolve. The key idea is that strategies will be imitated depending on the relative utility derived from each strategy. In our model, cooperators restrain themselves to a socially optimal level of water extraction and punish defectors who extract water at an individually optimal level. 
Our model builds upon Sethi \& Somanathan (1996) regarding how the social norm of restraining water and punishing uncooperative behavior evolves. A key difference is that Sethi \& Somanathan (1996) use a deterministic model building on ordinary differential equations, while we develop a probabilistic agent-based model.

Punishment takes place upon encounters between two agents. We model encounters as a Poisson processes (Richter et al. 2013), meaning that encounters occur randomly between two agents. The probability that agent $i$ is part of such encounter is thus equal to $2 / N$. At time $t$ in total $k$ encounters take place in the community, so that agent $i$ has an expected number of encounters equal to $(2 / N) k$. Denoting $\lambda=2 k / N$, we define $\lambda$ as the community social capital which indicates how frequently an agent encounters others in the community. For example, when $k=N$, then $\lambda=2$, meaning that an agent has a chance of encountering at least two other agents in the community at time $t$.

If a cooperator and a defector encounter each other, social sanctions, i.e. punishments occur. We assume that a cooperator incurs a utility loss $\mu$ from punishing a defector who, in turn, bears a utility loss $\omega$ from being punished. We refer to $\mu$ and $\omega$ as a unit cost of punishing and being punished respectively for cooperators and defectors and assume $\mu<\omega$. This can be thought of as social disapproval or actual destruction of material (Masclet et al. 2003). The expected utility loss of a cooperator from sanctioning defectors is thus increasing with the number of defectors being caught and punished. The expected utility loss of a defector from being punished is increasing with the number of cooperators imposing punishment. Punishment is probabilistic, i.e. a cooperator may punish more than one agent and a defector may be punished by more than one cooperator.

In each interaction loop, the number of social encounters are counted. For example, the number of defectors being caught and punished by an agent $i$ (a cooperator) at time $t$ is given by $D_{t, i}$, while the number of cooperators imposing punishment on individual agent $j$ (a defector) at time $t$ is given by $C_{t, j}$. The utility of cooperator $i\left(U_{i}^{C}\right)$ and defector $j\left(U_{j}^{D}\right)$ are, thus, given by

$$
\begin{aligned}
& U_{t, i}^{C}=\pi_{t, i}^{C}-\mu D_{t, i}, \\
& U_{t, j}^{D}=\pi_{t, j}^{D}-\omega C_{t, j} .
\end{aligned}
$$

An agent who bears excessive punishment costs (either as a punisher or as being punished) considers changing behavior. Whether agents change behavior depends on how successful the 
current strategy is compared to what others in the community are doing. Again, this process is random. We assume that - after all punishment has taken place - two agents are matched randomly. The probability of switching to the other strategy depends on how successful both strategies are. If the utility of agent $i$ is lower than that of agent $j$, the probability of agent $i$ switching from strategy $i$ to $j$ being equal to $\left(U_{j}-U_{i}\right) /\left(U_{j}+U_{i}\right)$. Otherwise, the agent $i$ keeps using the same strategy.

For the analysis in the next section, three key parameters are chosen, namely the initial proportion of cooperators $\left(C_{o}\right)$, punishment strength $(\omega / \mu)$ which depicts how costly it is for an agent to be punished for violating norms, and social capital $(\lambda=2 k / N)$ which depicts how likely and frequently agents encounter and perform punishment in a single year.

\subsection{Results}

The agent-based model is solved for the given parameter values by MATLAB (Mathworks Inc.). All parameter values and their definitions are summarized in Table 1. In section 3.1-3.2 we illustrate the effect of changing water availability and variability on cooperation in cases where agents are homogeneous in terms of yields and wealth. In section 3.3 we analyze whether cooperation can be maintained when agents differ in terms of skills and land endowments. For the same amount of water use, agents possessing high skill can produce higher yield than those who possess low skill. Similarly, agents endowed with large landholding can generate more income than those endowed with small landholding, as income is directly proportional to land. 
Table 1. Model variables and parameters with default values

\begin{tabular}{|c|c|c|c|c|}
\hline Symbol & Definition & \multicolumn{2}{|l|}{ Values } & Unit \\
\hline \multicolumn{5}{|l|}{ Variables } \\
\hline$Q$ & \multicolumn{3}{|l|}{ Total water available } & $\mathrm{m}^{3}$ \\
\hline$w$ & \multicolumn{3}{|l|}{ Individual water withdrawal } & $\mathrm{m}^{3} / \mathrm{ha}$ \\
\hline$W$ & \multicolumn{3}{|l|}{ Total water withdrawal } & $\mathrm{m}^{3}$ \\
\hline C & \multicolumn{4}{|l|}{ Number of cooperators } \\
\hline$D$ & \multicolumn{4}{|l|}{ Number of defectors } \\
\hline \multicolumn{2}{|l|}{ Parameters } & Rainy season & Dry season & \\
\hline $\bar{Q}$ & Mean inflow & 700000 & & $\mathrm{~m}^{3}$ \\
\hline$\sigma_{Q}$ & Standard deviation of inflow & 200000 & & $\mathrm{~m}^{3}$ \\
\hline$\alpha$ & Yield parameter & 0 & 0 & $\mathrm{~kg} / \mathrm{ha}$ \\
\hline$\beta$ & Yield parameter & 2 & 2.5 & $\mathrm{~kg} / \mathrm{m}^{3}$ \\
\hline$\gamma$ & Yield parameter & $1 / 1500$ & $1 / 1500$ & {$\left[\mathrm{~kg} / \mathrm{m}^{3}\right] \cdot\left[\mathrm{m}^{3} / \mathrm{ha}\right]^{-1}$} \\
\hline$p$ & Unit sale price & 0.25 & 0.3 & $\$ / \mathrm{kg}$ \\
\hline$c$ & Fixed cost per unit land & 200 & 200 & \$/ha \\
\hline $\bar{a}$ & Mean acreage & 3 & 3 & ha \\
\hline $\bar{a}_{S}$ & Mean acreage of small landholders & 2 & 2 & ha \\
\hline $\bar{a}_{L}$ & Mean acreage of large landholders & 4 & 4 & ha \\
\hline$\sigma_{a, S}, \sigma_{a, L}$ & Standard deviations of acreage & 1 & 1 & ha \\
\hline$C_{o}$ & Initial proportion of cooperators & 0.5 & & \\
\hline$N$ & Community size & 100 & & \\
\hline$\lambda$ & Social capital & 1 & & \\
\hline$\mu$ & Unit cost of punishing & 400 & & $\$$ \\
\hline$\omega$ & Unit cost of being punished & 600 & & $\$$ \\
\hline $\bar{\rho}$ & Average skill factor & 1 & & \\
\hline
\end{tabular}

\subsubsection{Dynamic patterns of emergence and collapse of cooperation}

We started the simulation with default value of parameters except that of punishment strength and observed temporal patterns of cooperation under two conditions: low punishment strength $(\omega / \mu=1.2)$ and high punishment strength $(\omega / \mu=3.5)$, as peer punishment is the mechanism in our model that may sustain cooperation. High punishment strength means it is very costly to defect. Through 100 repeated runs, we observed that the model reaches only two possible equilibria: full cooperation (full-C) and full defection (full-D). Mixed equilibria comprising 
cooperators and defectors were not found, consistent with the results of Sethi \& Somanathan (1996). For a certain range of parameter values (see section 3.2), however, the model could reach bi-stability where the system rests in either full-C equilibrium or full-D equilibrium, depending on stochastic dynamics. In general, the model reaches the equilibrium well before the $100^{\text {th }}$ time step. When agent heterogeneity is considered, however, equilibrium time varies. For each repeated run, we thus run the model until it reaches equilibrium. In Fig. 2, we show the two possible outcomes where cooperation collapses under the low punishment condition and emerges under the high punishment condition. The collapse of cooperation (Fig. 2a) is preceded by an widening gap in utility between cooperators and defectors with defectors' utility being higher. In a similar vein, the emergence of cooperation is followed by an increasing utility of cooperators over utility of defectors.
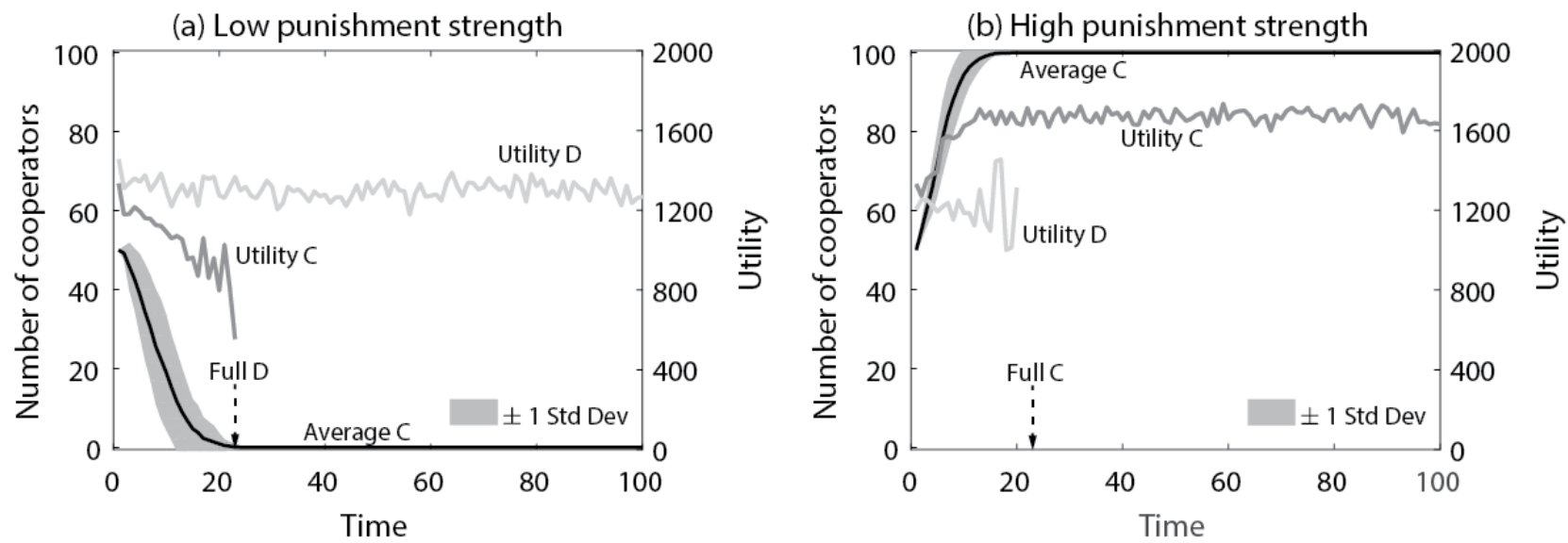

Fig. 2. Temporal patterns of emergence and collapse of cooperation under a) low punishment condition $(\omega / \mu=1.2)$ and $b)$ high punishment condition $(\omega / \mu=3.5)$. The model was simulated with default parameter values for 100 repeated runs. The number of cooperators at each time step and the utility of defectors (D) and cooperators (C) were averaged over 100 runs, where standard deviations were also shown.

\subsubsection{Effects of key social, economic and environmental parameters on cooperation}

We conduct a series of simulations to analyze how key social, economic and environmental parameters affect cooperation. The model reaches only two possible equilibria - full cooperation (full-C) and full defection (full-D) - depending on social and environmental parameters (Fig. 3). 
First, as the punishment strength $(\omega / \mu)$ increases - which measures how costly it is for a defector to violate the norm, the percentage of runs that reach full-C increases. When $\omega / \mu>$ 2.0, all simulations result in full-C. Intuitively, if the costs of punishing free-riders decrease visa-vis the costs of being punished for free-riding, social norms of cooperation spread more easily in the community. Second, the greater the initial proportion of $\mathrm{C}$, the higher the percentage of runs that reach the full-C equilibrium. Intuitively, if only a small number of cooperators attempt to discipline many defectors, they will quickly give up - leading to the full-D equilibrium. At the same time, many cooperators will be very successful in punishing a small number of defectors, leading to the full-C equilibrium. If the initial proportion of $\mathrm{C}$ is neither too small nor too large $\left(0.4<C_{0}<0.6\right)$, the model may reach either full-C or full-D equilibrium, giving rise to bistability. Third, increasing the relative price $\left(P_{d} / P_{r}\right)$, which measures the benefits of saving water for the dry season, leads to less cooperation. Tragically, when cooperation is most beneficial, least cooperation is observed. Intuitively, this happens because cooperators are doing what is socially optimal (saving more water), while the defectors are the ones who benefit, increasing the temptation to defect. Fourth, social capital $\lambda$, which measures the frequency of encounters in the community, has a positive effect on cooperation. Intuitively, more interactions imply that defection is detected and sanctioned more often, disciplining defectors. Finally, an increasing water inflow increases the chance of reaching full cooperation, as more water can potentially be saved for use in the dry season, decreasing the incentive for defecting. Increasing resource variability favors defection, mostly because in times of scarcity cooperators are the ones who restrain water use, increasing the temptation to defect. 
(a)

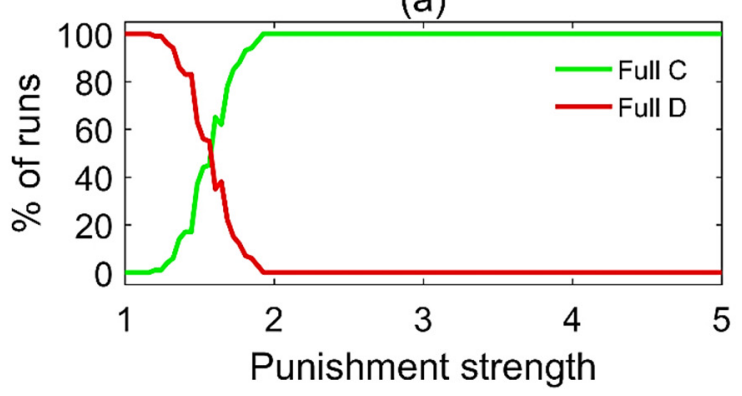

(c)

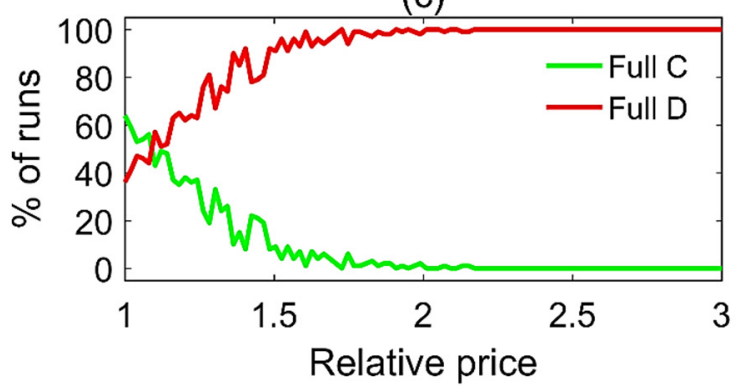

(e)

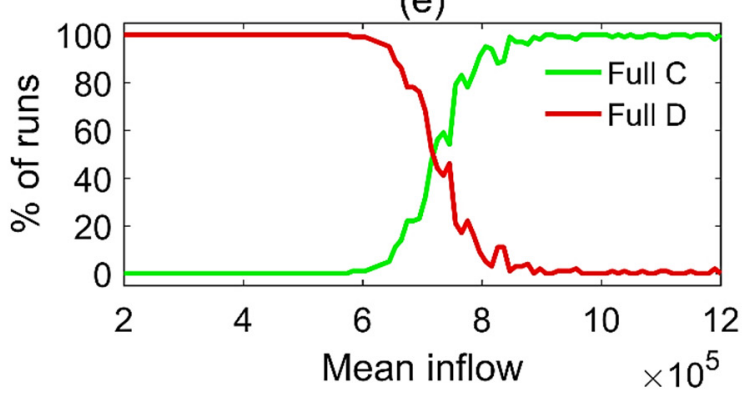

(b)

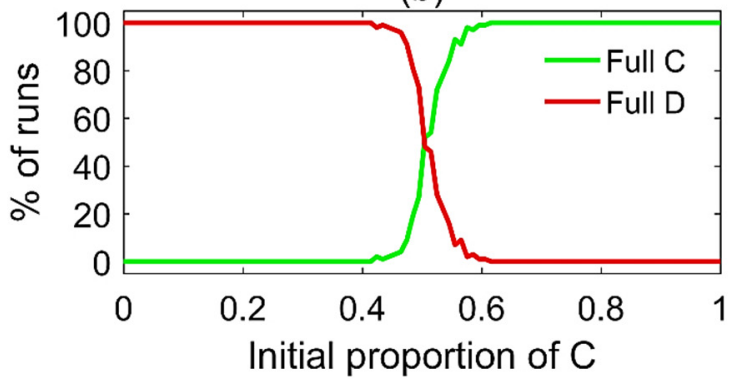

(d)

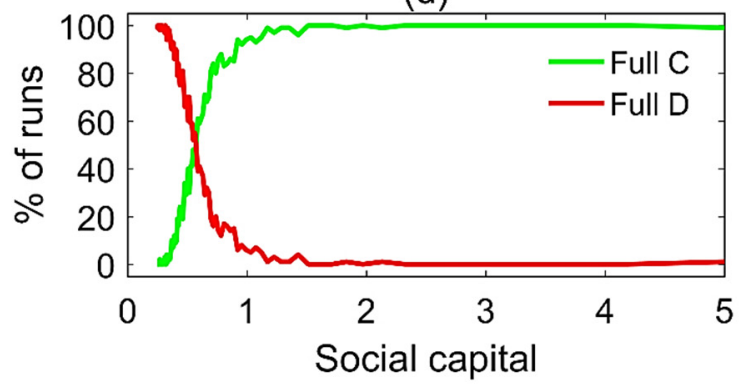

(f)

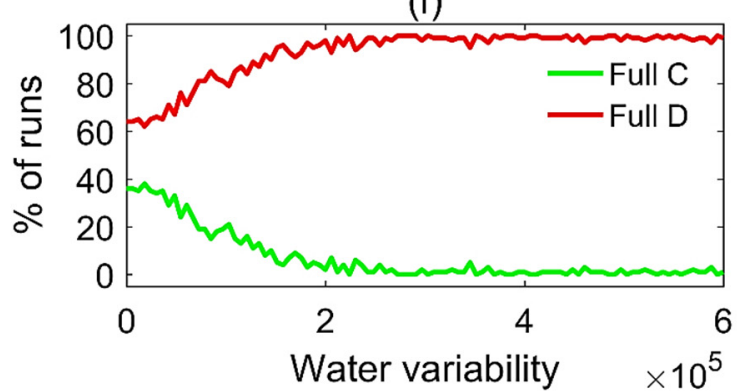

Fig. 3. We present the effect of key parameters on cooperation in the form of one-at-a-time sensitivity plots where the $x$-axis stands for the varying values of a) punishment strength, b) initial proportion of cooperators, c) relative sales price, d) social capital, e) mean water inflow, f) water variability. To account for stochasticity in the model, we run the model 100 times repeatedly for each single value of the parameter and count the percentage of model runs that reach the full-cooperation equilibrium (green line) and full-defection equilibrium (red line).

\subsubsection{Effects of changing resource conditions}

\section{(a) Effects of resource scarcity}

In Fig. 4 we show that increasing water scarcity may lead to the collapse of cooperation in various social and economic circumstances. Overall, under the scarcity condition where the mean inflow is less than $7 \times 10^{5}$, full defection is the outcome for a large parameter space of the initial proportion of cooperators (Fig. 4a), punishment strength (Fig. 4b), social capital (Fig. 4c) and relative price (Fig. 4d). In all those cases, cooperation collapses because the water that 
is saved by cooperators for potential use in the dry season is taken out by defectors, which makes the profits of the latter relatively higher than the former. If water is abundant, cooperation can thrive for most of the parameter space for all social and economic parameters. Here, when water is almost sufficiently available for all agents for farming in both seasons, the difference between the profits of cooperators and defectors is small, making the defecting strategy inferior as the utility loss due to punishment is larger than the potential gains from using slightly more water.

(a)

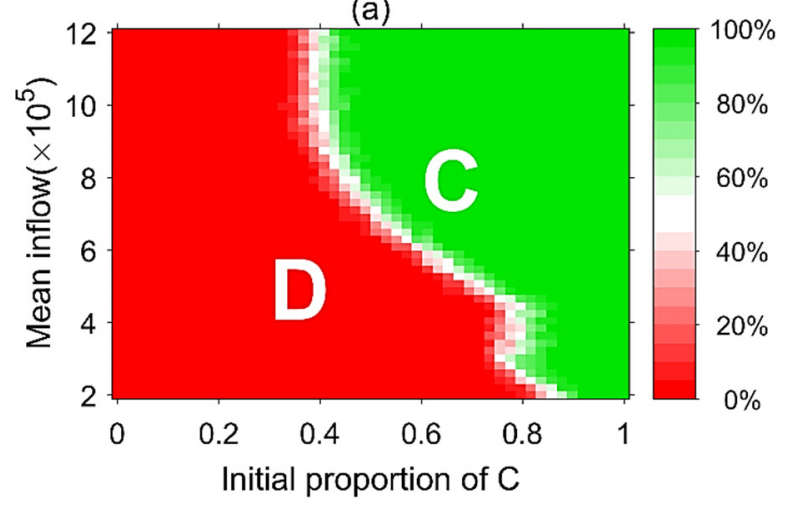

(c)

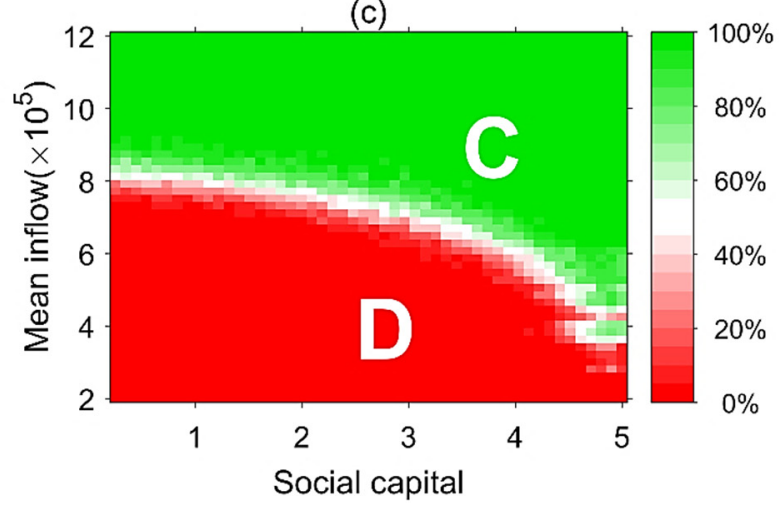

(b)

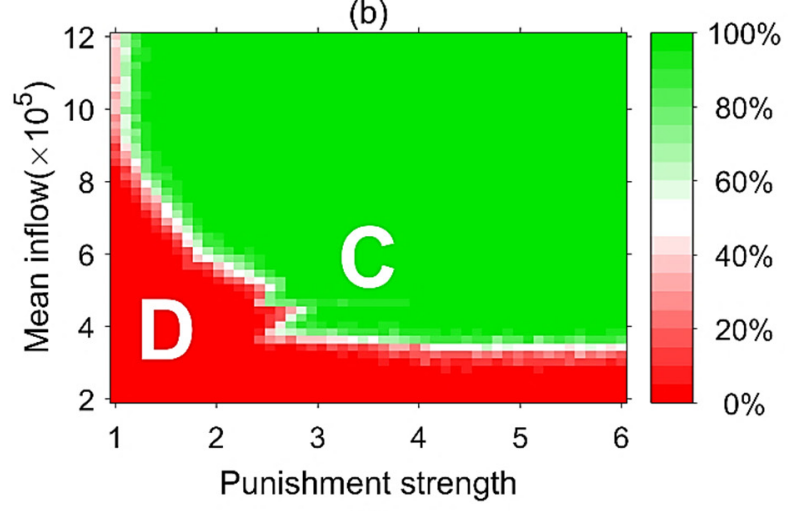

(d)

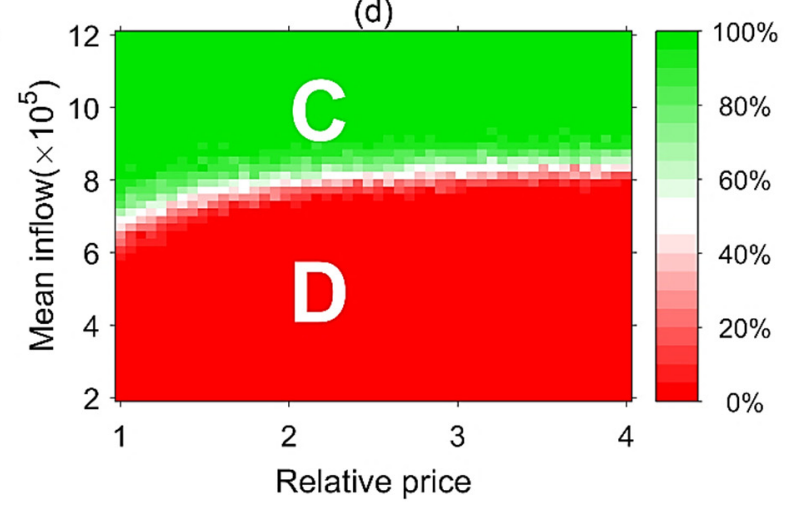

Fig. 4. Heat map illustrating the effects of mean water inflow on cooperation for key social and economic parameters. The lower the mean inflow is compared to the default value $\left(7 \times 10^{5}\right)$, the greater the scarcity is. Here, we performed 100 repeated runs for each single value of the parameters and count the percentage of model runs that reach the full-cooperation or the full-defection equilibrium. The color bar shows the percentage of model runs that reach full-cooperation equilibrium (light green) and fulldefection equilibrium (red). The white color represent the case where the model features bistability and may reach either full cooperation or full defection.

\section{(b) Effects of resource variability}

In Fig. 5 we show that variation in water inflow has a small negative effect on cooperation under various social and economic conditions. If the water inflow varies widely from year to year, it is more difficult to maintain cooperation in the community. For almost the whole 
parameter space for the initial proportion of cooperators, social capital and relative price, the defectors dominate in the community, especially when the degree of variability is high $\left(\sigma_{Q}>\right.$ $\left.2 \times 10^{5}\right)$. Cooperation can be maintained, however, if the punishment strength is high $(\omega / \mu>$ 2). Note that water variability has a much weaker effect on cooperation than changes in mean inflow. Intuitively, a high degree of water variability leads to a reduction in profits of both cooperators and defectors. However, as the profit function is concave in water use, a high variability slightly favors defection. Also, increased variability increases the zone of bistability, as stochasticity is more pronounced.

(a)

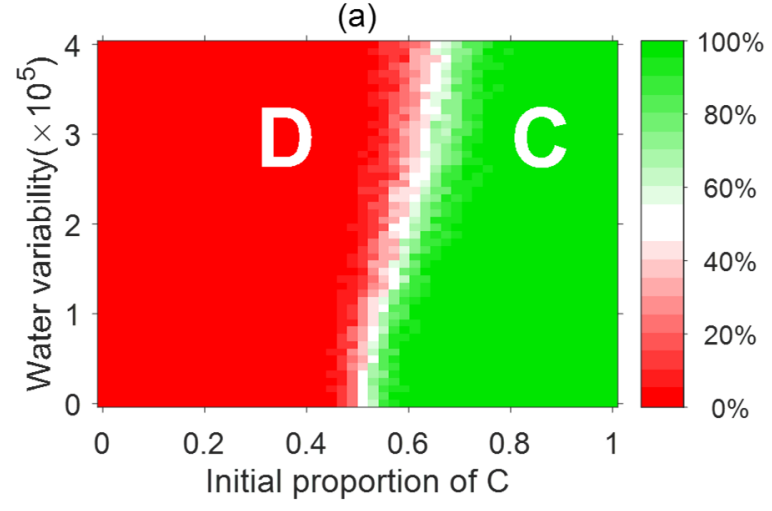

(c)

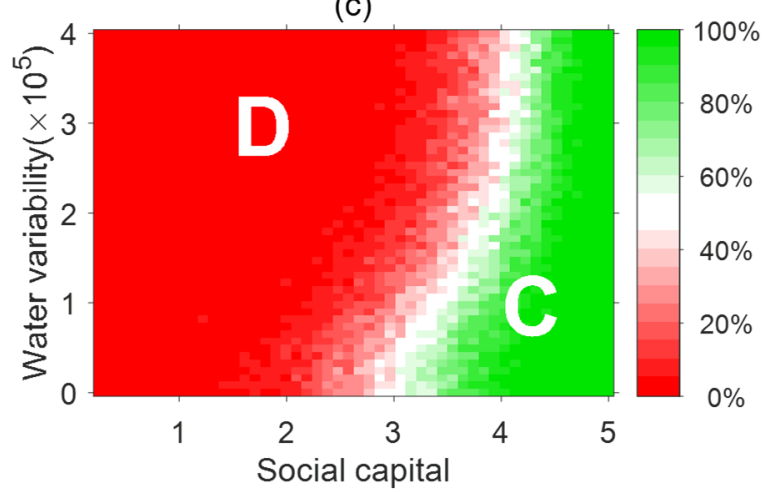

(b)

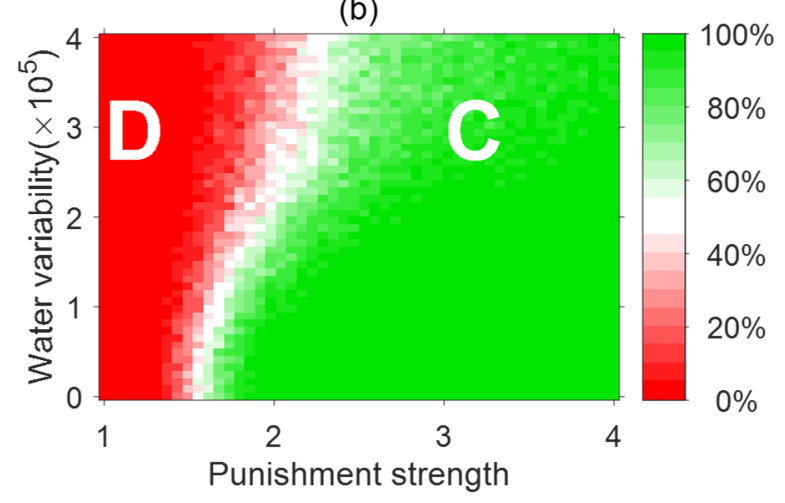

(d)

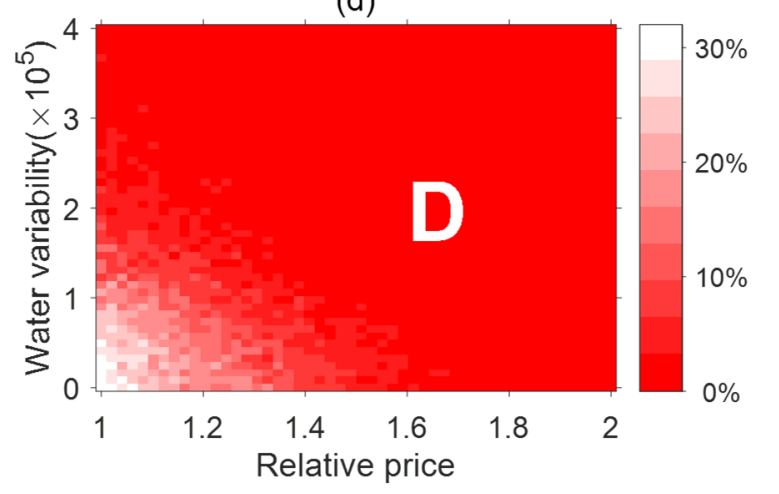

Fig. 5. Heat map illustrating the effects of water variability on cooperation for key social and economic parameters. The higher the standard deviation of resource inflow is compared to its default value $\left(2 \times 10^{5}\right)$, the more variable the resource is from year to year. To account for stochasticity, we performed 100 repeated runs for each single value of the parameters and count the percentage of model run that reach full-cooperation or full-defection equilibrium. The color bar shows the percentage of model runs that reach full-cooperation equilibrium (light green) and full-defection equilibrium (red). The white color represent the case where the model features bistability and may reach either full cooperation or full defection. 


\section{(c) Combined effects of resource scarcity and variability}

So far we have explained the effects of water scarcity and water variability on cooperation separately. Here, we consider the case where water scarcity and water variability may interact and affect cooperation in the community. Fig. 6 shows that the combined effects of water scarcity and water variability on cooperation become much more pronounced when the two interact. If punishment strength and social capital are low, it is impossible to maintain cooperation under the condition of highly variable inflow $\left(\sigma_{Q}>4 \times 10^{5}\right)$, even if water is abundant $\left(\bar{Q}>10 \times 10^{5}\right)$ (Fig. 6a). Cooperation is only stable when the mean inflow is high and the degree of variability is low. However, if the punishment strength is high (Fig. 6b), cooperation can be enhanced, even if social capital is low and water is scarce, as long as the degree of water variability is not too high. Furthermore, if punishment is low and the community has strong social capital, cooperation can be maintained in various levels of water scarcity and variability (Fig. 6c). Finally, under high social capital and high punishments cooperation can be supported, unless the mean inflow is very low $\left(\bar{Q}<3 \times 10^{5}\right)$

(a) Low punishment, Low social capital

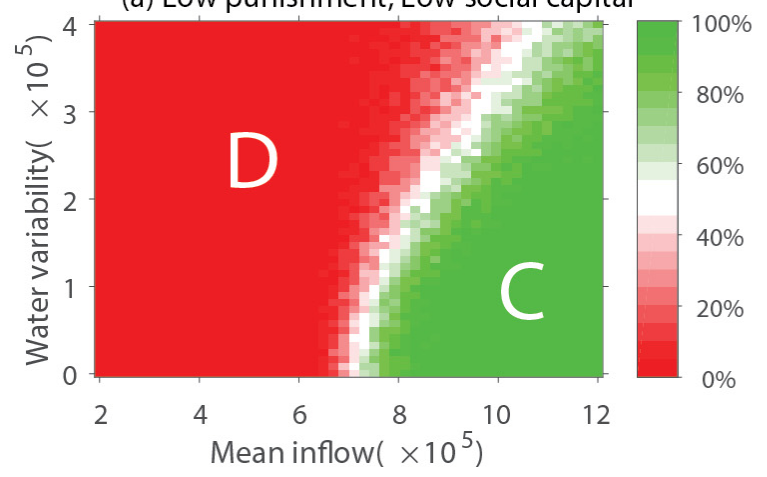

(c) Low punishment, High social capital

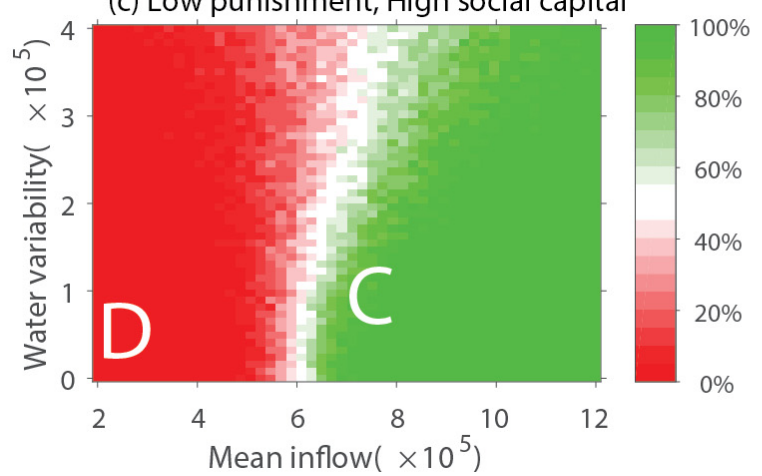

(b) High punishment, Low social capital

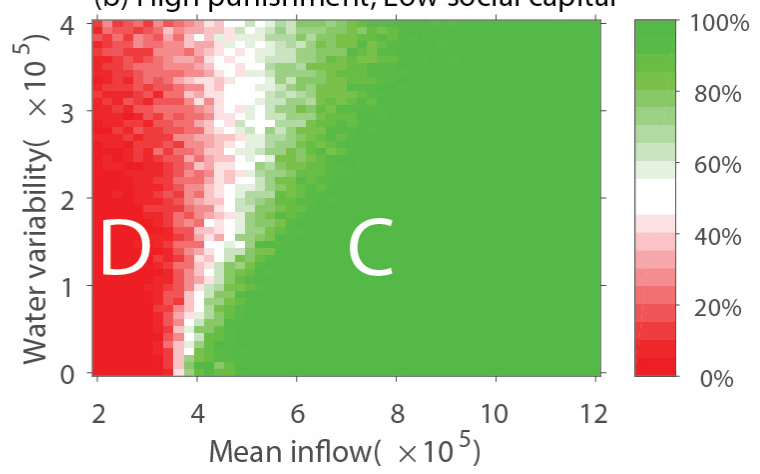

(d) High punishment, High social capital

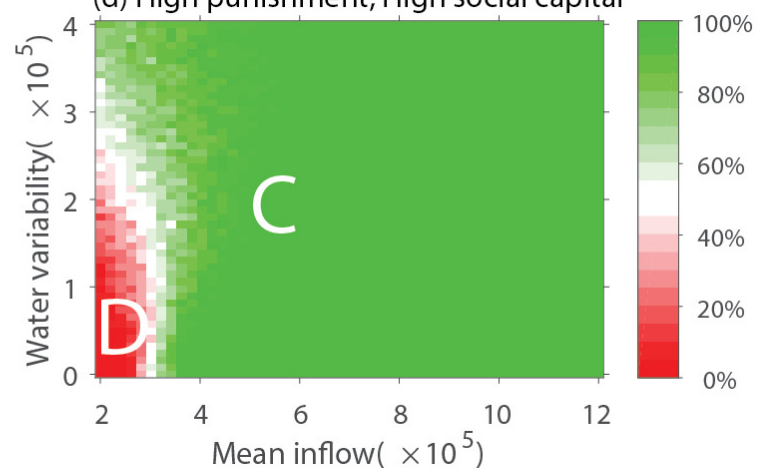

Fig. 6. Combined effects of water scarcity and variability on cooperation for a) low punishment and low social capital, b) high punishment and low social capital, c) low punishment and high social capital, d) high punishment and high social capital. To account for stochasticity, we performed 100 repeated runs for each single value of the parameters and count the percentage of model run that reach the full- 
cooperation or full-defection equilibrium. The color bar shows the percentage of model runs that reach full-cooperation equilibrium (light green) and full-defection equilibrium (red). The white color represent the case where the model may reach either full cooperation or full defection.

\subsubsection{Effects of inequality and heterogeneity}

\section{(a) Skill heterogeneity}

In the previous section we have analyzed water scarcity and water variability without considering different capacities or skills of farmers. Here, we observe in Fig. 7 that skill heterogeneity has only a weak effect on cooperation. For instance, if water becomes scarcer, cooperation will collapse regardless of the degree of heterogeneity (Fig. 7a). In addition, if water varies greatly from year to year, skill heterogeneity reduces the probability of reaching full cooperation. (Fig. 7b). In our model, a more skillful agent is able to produce higher yield than a low-skill agent for the same amount of water use. Intuitively, cooperation is affected because heterogeneous skills blur the relationship between decisions and outcomes. A low-skill defector is potentially worse off even if subjected to mild punishment, while a very skillful defector may be able to succeed in spite of large social disapproval, even though he would have been outperformed by cooperators if skills were homogenous.

(a)

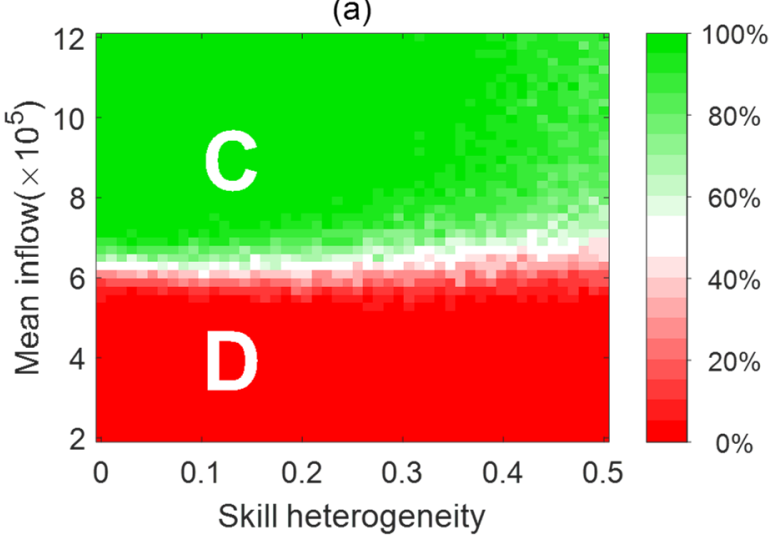

(b)

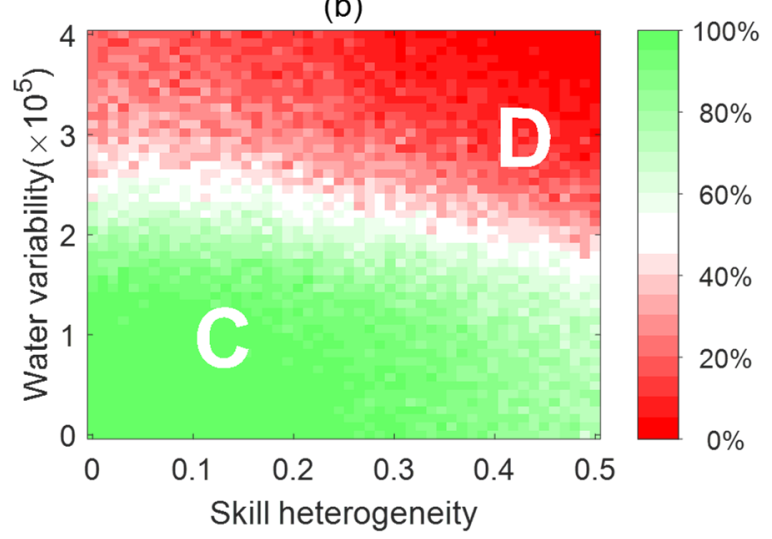

Fig. 7. The combined effects of skill heterogeneity and environmental changes on cooperation. Here, the $\mathrm{x}$-axis represents the varying values of standard deviation of the skill parameter - the higher the value, the higher the degree of heterogeneity. The y-axis represents the environmental parameters, namely a) the mean inflow and b)water variability. Here, we performed 100 repeated runs for each single value of the parameters and count the percentage of model runs that reach the full-cooperation or fulldefection equilibrium. The color bar shows the percentage of model runs that reach full-cooperation equilibrium (light green) and the full-defection equilibrium (red). The white color represent the case where the model may reach either full cooperation or full defection. 


\section{(b) Land inequality}

Land inequality is another potential factor that can catalyze the effects of environmental changes on cooperation. We model inequality as the degree of variability in land distribution among agents (land heterogeneity) and proportion of large landholders in the community. Overall, increasing inequality can lead to a collapse of cooperation, especially under conditions of low water inflow and high degree of water variability (Fig. 8). Cooperation can only emerge if low degree of land heterogeneity goes hand in hand with i) a very high degree of water inflow (Fig. 8a) or ii) a very low degree of water variability (Fig. 8c). In our model, profit is directly proportional to land, a more unequal distribution of land results in a greater discrepancy in profits as well as utility among agents, making it harder for cooperators to discipline defectors. Intuitively, inequality makes sanctioning less effective, because high-earning defectors may still enjoy relatively higher utility than poor cooperators, inducing the latter group to also defect . Similarly, increasing the proportion of large landholders can potentially reinforce the negative effects of water scarcity on cooperation (Fig. $8 \mathrm{~b}$ and $8 \mathrm{~d}$ ). Intuitively, similar to the effects of land heterogeneity, large landowners will not feel sanctions sufficiently strongly to change behavior, and less fortunate individuals may end up imitating them. For example, a poor farmer with small landholding may imitate a rich farmer with large landholding regarding the decision on water use, hoping to be as successful as the rich one. The inability to separate the role of extra income gained from cultivating on a relatively large land size, can be illusive for the poor as the foundation of the success (i.e. land endowments) cannot be imitated. 
(a)

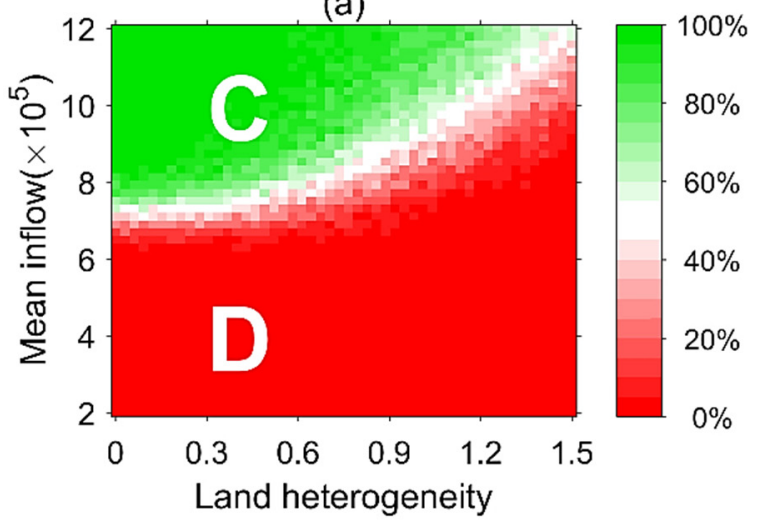

(c)

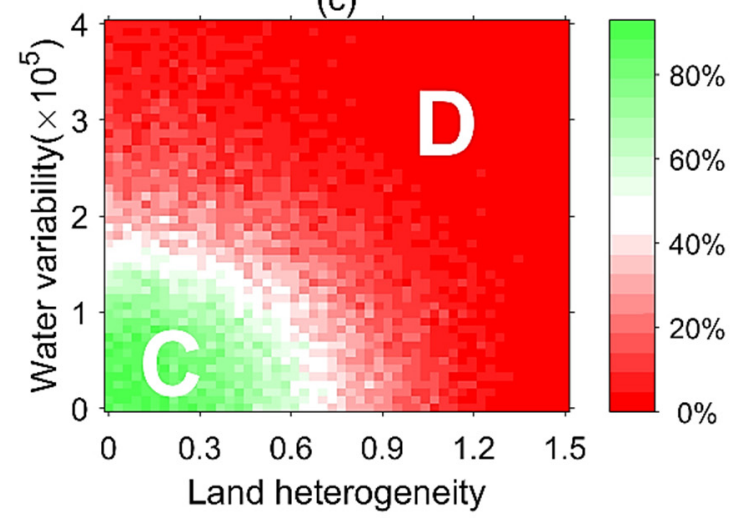

(b)

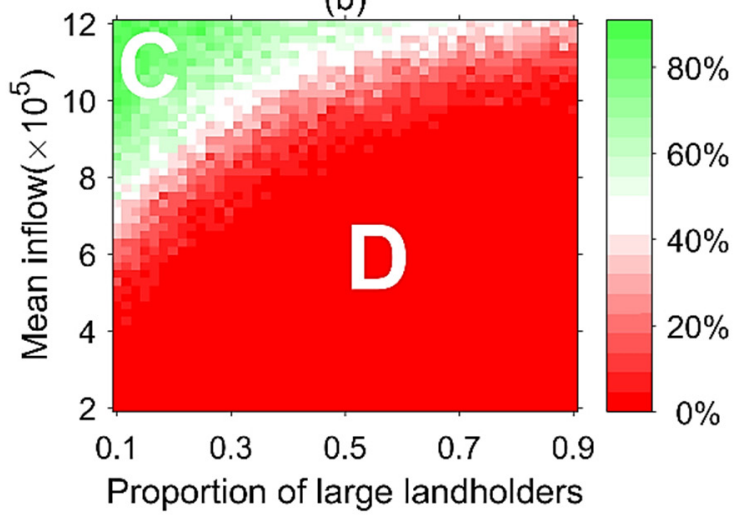

(d)

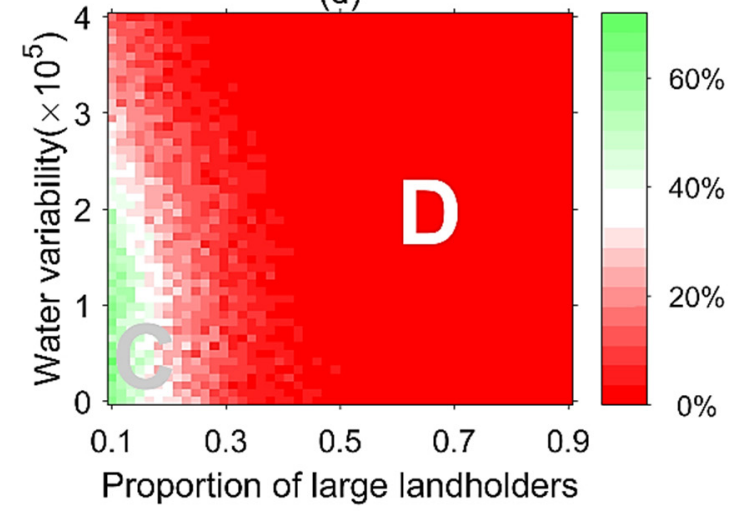

Fig. 8. The combined effects of land inequality and environmental changes on cooperation. Here, the xaxis represents the varying values of land heterogeneity which is characterized by the standard deviation of land and proportion of large landholders. The y-axis represents the environmental parameters, namely the mean inflow and the water variability. Here, we performed 100 repeated runs for each single value of the parameters and count the percentage of model run that reach the full-cooperation or full-defection equilibrium. The color bar shows the percentage of model runs that reach full-cooperation equilibrium (light green) and the full-defection equilibrium (red). The white color represent the case where the model may reach either full cooperation or full defection.

\subsection{Discussions and conclusion}

We have developed an agent-based model to analyze how socioeconomic and environmental changes affect the cooperation of agents in a self-governing community who share a resource. Our model considers a community of resource users who share water from a joint river. The water availability varies between seasons and between years and the members may be exposed to some degree of heterogeneity in terms of skills and land endowments. Social norms of restraining water use are emerging in the community and punishment of norm-violators is the enforcement mechanism to overcome the social dilemma arising from seasonal allocation of water. 
The model results show that social norms may erode in response to changes in resource conditions. Particularly, we have shown that resource scarcity and variability may hinder cooperation, especially when combined, and when the community faces some degree of inequality and heterogeneity. These findings are consistent with those of Sethi \& Somanathan (1996), who show that cooperation can be maintained if the number of punishers is sufficiently large, but upon collapse, cooperation cannot reemerge. Our model predictions differ from Schlüter et al. (2016), who found that scarcity may actually foster cooperation. The difference comes from the assumptions regarding performance under scarcity and abundance. While in Schlüter et al. (2016), the benefits from defecting are relatively smaller under scarcity, in our model defecting is especially lucrative under scarcity because of the presence of rainy and dry seasons. Any attempts taken by cooperators to save water for the dry season are benefitting defectors, who may use the water in the rainy season already. On a more fundamental level, the question whether cooperation thrives or erodes in times of scarcity, depends on how (i) the benefits and costs of cooperative acts are distributed and (ii) the enforcement mechanism in place. In the real world, both elements will be case-specific and therefore the question of cooperation will be mostly an empirical, rather than a theoretical one. However, with a theoretical model like this one can produce hypotheses that will then provide good grist for our empirical mills to test key factors that facilitate or undermine cooperation in the field.

Our theoretical model is based on evolutionary game theory where agents are assumed to act cooperatively or selfishly following the tradition of Sethi and Somanathan (1996). The behavioral rules are still relatively simple and it would be interesting to add more realism to a theoretical model like this one. First, in our model the only available strategies are cooperation and defection. It would be very interesting to allow for continuous strategies, where agents could choose from a whole continuum of extraction levels and cooperation would not be an all or nothing decision (Killingback \& Doebeli 2002; Doebeli et al. 2004). Second, we assumed that the community is well-mixed, i.e. encounters with other agents are entirely random. Allowing for spatial structure, either as people having neighbours (Nowak 2006b; Noailly et al. 2009) or operating in a network (Rand et al. 2014; Ohtsuki et al. 2006) would most likely create cooperative clusters and favor co-existence of cooperators and defectors. Third, we assume that the remaining water in the dry season is divided symmetrically among all agents. This assumption is obviously motivated by analytical convenience, rather than realism. Alternatively, one could consider that skillful get more water, since they can use it more efficiently. Also, it seems plausible that richer farmers are more successful in appropriating 
water, potential increasing the income difference between rich and poor farmers. Fourth, punishment is assumed to be only dependent on the number of cooperators, but the punishment costs are constant for punishers and defectors. In reality, both components are most likely dependent on how widespread defection is, as it seems much more difficult to sanction selfish behavior if it is in line with the empirical, i.e. observed social norm. Also, it seems plausible that users are more inclined to sanction if the resource is scarce and the social dilemma is more severe. Such adaptive punishment may be able to respond to scarcity and potentially also to the erosion of norms itself, either requiring stronger punishment or changing the cooperative strategy itself under scarcity. Richter \& Dakos (2015) have shown that such collapse of norms can be anticipated with resilience indicators, derived from, for example, fluctuations in profits. Whether such adaptive self-governance system could evolve fast enough - if at all - and how it would look like seems like an exciting topic for further research.

While our study is theoretically grounded, our paper provides some indications for the fragility of cooperation towards external pressures, such as climatic changes. In the context developed here, we have shown that the resilience of social norms could be weakened by those changes and a collapse of cooperative arrangements may occur. For policy makers, an important takehome message is that any projected changes in agricultural yields due to climate change will be dependent on how the institutional setting responds to scarcity. Our results are purely theoretical, but they suggest that welfare losses because of climate change might be higher than expected, as this would not only depend on water availability, but also on the welfare losses that may arise from the breakdown of cooperative arrangements.

While the paper mainly shows the fragility of social norms, there are also promising findings. Perhaps most importantly, cooperation is much more resilient towards external pressures if social capital (i.e. the frequency of encounters) is high. Fostering community meetings and creating common interaction places (e.g. common drying areas) seems like a relatively cheap option to maintain cooperation. Such approaches are indeed often favored by NGOs (Agrawal \& Gibson 1999). Our model is entirely theoretical and its lack of empirical grounding prevents any predictions about real world cases. Therefore, an exciting next step would be to take our model predictions to the field to validate the results and also test potential policy solutions to foster the resilience of social norms that help preserving common-pool resources. 


\section{References}

Agrawal, A. \& Gibson, C.C., 1999. Enchantment and disenchantment: the role of community in natural resource conservation. World Development, 27(4), pp.629-649.

An, L. et al., 2014. Agent-based modeling in coupled human and natural systems (CHANS): lessons from a comparative analysis. Annals of the Association of American Geographers, 104(4), pp.723-745.

An, L., 2012. Modeling human decisions in coupled human and natural systems: Review of agent-based models. Ecological Modelling, 229, pp.25-36.

Bausch, A.W., 2014. Evolving intergroup cooperation. Computational and Mathematical Organization Theory, 20(4), pp.369-393.

Berger, T., 2001. Agent-based spatial models applied to agriculture: a simulation tool for technology diffusion, resource use changes and policy analysis. Agricultural Economics, 25(2-3), pp.245-260.

Bisaro, A. \& Hinkel, J., 2016. Governance of social dilemmas in climate change adaptation. Nature Climate Change, 6(4), pp.354-359.

Blanco, E., Lopez, M.C. \& Villamayor-Tomas, S., 2015. Exogenous degradation in the commons: field experimental evidence. Ecological Economics, 120, pp.430-439.

Bonabeau, E., 2002. Agent-based modeling: methods and techniques for simulating human systems. Proceedings of the National Academy of Sciences, 99(suppl. 3), pp.7280-7287.

Brandt, G. \& Merico, A., 2013. Tipping points and user-resource system collapse in a simple model of evolutionary dynamics. Ecological Complexity, 13, pp.46-52.

Doebeli, M., Hauert, C. \& Killingback, T., 2004. The evolutionary origin of cooperators and defectors. Science, 306(5697), pp.859-862.

Elliott, J. et al., 2014. Constraints and potentials of future irrigation water availability on agricultural production under climate change. Proceedings of the National Academy of Sciences, 111(9), pp.3239-44.

Esteve, P. et al., 2015. A hydro-economic model for the assessment of climate change impacts and adaptation in irrigated agriculture. Ecological Economics, 120, pp.49-58.

Gardner, R., Ostrom, E. \& Walker, J.M., 1990. The nature of common-pool resource problems. Rationality and Society, 2(3), pp.335-358.

Gavrilets, S. \& Richerson, P.J., 2017. Collective action and the evolution of social norm internalization. Proceedings of the National Academy of Sciences, 114(23), pp.6068- 
6073.

Grossman, H.I. \& Mendoza, J., 2003. Scarcity and appropriative competition. European Journal of Political Economy, 19(4), pp.747-758.

Haddeland, I. et al., 2014. Global water resources affected by human interventions and climate change. Proceedings of the National Academy of Sciences, 111(9), pp.32513256.

Hassani-Mahmooei, B. \& Parris, B.W., 2013. Resource scarcity, effort allocation and environmental security: an agent-based theoretical approach. Economic Modelling, 30(1), pp.183-192.

Heath, B., Hill, R. \& Ciarallo, F., 2009. A survey of agent-based modeling practices (January 1998 to July 2008). Journal of Artificial Societies and Social Simulation, 12(4), p.9.

Heckbert, S., Baynes, T. \& Reeson, A., 2010. Agent-based modeling in ecological economics. Annals of the New York Academy of Sciences, 1185(1), pp.39-53.

Jaeger, W.K. et al., 2017. Finding water scarcity amid abundance using human-natural system models. Proceedings of the National Academy of Sciences, 114(45), pp.1188411889.

Janssen, M.A. \& Ostrom, E., 2007. Adoption of a new regulation for the governance of common- cool resources by a heterogeneous population. In J.-M. Baland, P. K. Bardhan, \& S. Bowles, eds. Inequality, Cooperation, and Environmental Sustainability. Princeton University Press, pp. 60-96.

Killingback, T. \& Doebeli, M., 2002. The continuous prisoner's dilemma and the evolution of cooperation through reciprocal altruism with variable investment. American Naturalist, 160(4), pp.421-438.

Kun, A. \& Dieckmann, U., 2013. Resource heterogeneity can facilitate cooperation. Nature communications, 4 , p.2453.

Levin, S. et al., 2012. Social-ecological systems as complex adaptive systems: modeling and policy implications. Environment and Development Economics, 18(2), pp.111-132.

Lewis, H.M. \& Dumbrell, A.J., 2013. Evolutionary games of cooperation: Insights through integration of theory and data. Ecological Complexity, 16, pp.20-30.

Liu, J. et al., 2007. Complexity of coupled human and natural systems. Science, 317(5844), pp.1513-1516.

Marchiori, C., 2014. Inequality and rules in the governance of water resources. Ecological Economics, 105, pp.124-129.

Masclet, D. et al., 2003. Monetary and nonmonetary punishment in the voluntary 
contributions mechanism. American Economic Review, 93(1), pp.366-380.

Noailly, J., Van den Bergh, J.C.J.M. \& Withagen, C.A., 2003. Evolution of harvesting strategies: replicator and resource dynamics. Journal of Evolutionary Economics, 13(2), pp.183-200.

Noailly, J., Bergh, J.C.J.M. \& Withagen, C.A., 2009. Local and global interactions in an evolutionary resource game. Computational Economics, 33(2), pp.155-173.

Nowak, M.A., 2006a. Evolutionary dynamics, Harvard University Press.

Nowak, M.A., 2006b. Five rules for the evolution of cooperation. Science, 314(5805), pp.1560-1563.

Nyborg, B.K. et al., 2016. Social norms as solutions. Science, 354(6308), pp.42-43.

Ohtsuki, H. et al., 2006. A simple rule for the evolution of cooperation on graphs and social networks. Nature, 441(7092), pp.502-505. Available at: http://www.nature.com/doifinder/10.1038/nature04605.

Osés-Eraso, N., Udina, F. \& Viladrich-Grau, M., 2008. Environmental versus human-induced scarcity in the commons: do they trigger the same response? Environmental and Resource Economics, 40(4), pp.529-550.

Osés-Eraso, N. \& Viladrich-Grau, M., 2007. On the sustainability of common property resources. Journal of Environmental Economics and Management, 53(3), pp.393-410.

Ostrom, E., 2009. A general framework for analyzing sustainability of social-ecological systems. Science, 325(5939), pp.419-422.

Ostrom, E., 1990. Governing the commons: the evolution of institutions for collective action, Cambridge University Press.

Ostrom, E., Gardner, R. \& Walker, J., 1994. Rules, games, and common-pool resources, University of Michigan Press.

Ostrom, E., Walker, J. \& Gardner, R., 1992. Covenants with and without a sword: selfgovernance is possible. American Political Science Review, 86(2), pp.404-417.

Pfaff, A. et al., 2015. Framed field experiment on resource scarcity \& extraction: pathdependent generosity within sequential water appropriation. Ecological Economics, 120, pp.416-429.

Rand, D.G. et al., 2014. Static network structure can stabilize human cooperation. Proceedings of the National Academy of Sciences, 111(48), pp.17093-17098.

Rasch, S. et al., 2016. Cooperation and collapse in a communal livestock production SES model - a case from South Africa. Environmental Modelling and Software, 75, pp.402413. 
Richter, A. \& Dakos, V., 2015. Profit fluctuations signal eroding resilience of natural resources. Ecological Economics, 117, pp.12-21.

Richter, A. \& Grasman, J., 2013. The transmission of sustainable harvesting norms when agents are conditionally cooperative. Ecological Economics, 93, pp.202-209.

Richter, A., van Soest, D. \& Grasman, J., 2013. Contagious cooperation, temptation, and ecosystem collapse. Journal of Environmental Economics and Management, 66(1), pp.141-158.

Schewe, J. et al., 2014. Multimodel assessment of water scarcity under climate change. Proceedings of the National Academy of Sciences, 111(9), pp.3245-3250.

Schlüter, M. et al., 2012. New horizons for managing the environment: a review of coupled social-ecological systems modeling. Natural Resource Modeling, 25(1), pp.219-272.

Schlüter, M. \& Pahl-Wostl, C., 2007. Mechanisms of resilience in common-pool resource management systems: an agent-based model of water use in a river basin. Ecology and Society, 12(2).

Schlüter, M., Tavoni, A. \& Levin, S., 2016. Robustness of norm-driven cooperation in the commons. Proc. R. Soc. B, 283(1822).

Sethi, R. \& Somanathan, E., 1996. The evolution of social norms in common property resource use. The American Economic Review, 86(4), pp.766-788.

Siekmann, I., 2015. Bifurcation analysis of individual-based models in population dynamics. Ecological Complexity, 21, pp.177-184.

Sigmund, K. \& Nowak, M.A., 1999. Evolutionary game theory. Current Biology, 9(14), pp.R503-R505.

Tavoni, A., Schlüter, M. \& Levin, S., 2012. The survival of the conformist: social pressure and renewable resource management. Journal of Theoretical Biology, 299, pp.152-161. 


\section{Authors' contribution}

T.N., A.R., and X.Z. designed the research. TN developed the model and carried out the analysis. All authors contributed to writing the final manuscript.

\section{Acknowledgments}

T.N. gratefully acknowledges funding from the European Commission through the Alfabet project under the EU Erasmus Mundus Program. 



\title{
Chapter 3-Path dependencies and institutional traps in water governance: Evidence from Cambodia
}

\author{
Tum Nhim ${ }^{1}$, Andries Richter ${ }^{1}$ \\ Environmental Economics and Natural Resources Group, Wageningen University \\ P.O. Box 8130, Wageningen 6700 EW, The Netherlands
}

Nhim, T. \& Richter, A. (2021). Path dependencies and institutional traps in water governance: Evidence from Cambodia. (under review).

\begin{abstract}
In many parts of the world, water governance is in the hands of local communities. Typically, social norms of cooperation are important mechanisms to ensure sufficient contributions to maintain a certain water infrastructure, and also to restrain excessive water use. Field work in Kampong Chhnang (Cambodia) documents a mixed picture regarding the success of selfgovernance. While some villages have well-functioning water infrastructure systems and high levels of cooperation, others are left with dysfunctional infrastructure and low cooperation. We hypothesize that this outcome may be the result of an institutional trap, where poor infrastructure leads to scarcity and low revenues, undermining cooperation further, essentially creating a vicious cycle. We also hypothesize that conditional cooperation may explain why some communities can overcome such an institutional trap. We develop an agent-based model, in which users have to decide how much to contribute to common water infrastructure and how much water to extract. This decision is based on economic considerations, but also reputational concerns, where the own decision is evaluated against the social norm, formalized as the mechanism of conditional cooperation. We find that the system features alternative stable states, depending on initial conditions. If the system has a functioning water system initially and a high level of cooperation, prosperity can be created, which facilitates further investments in water infrastructure, fostering cooperation further. If the community features initial scarcity, cooperation is relatively costly, undermining investments in water infrastructure.
\end{abstract}

Key words: Path dependencies, institutional traps, conditional cooperation, scarcity, agentbased modeling 


\subsection{Introduction}

In many parts of the world, water governance is in the hands of local communities (Ostrom 1990; Lansing et al. 2017). Social norms of cooperation have been identified as key mechanisms to ensure sufficient contributions to maintain a functioning water infrastructure, and also to restrain excessive water use. Yet, most studies on self-governance of common pool resources focus on either extraction of common-pool resources (CPR), such as water, or investment in public goods (PG) provisioning, such as water infrastructure, but rarely both combined. In many real-world situations, however, both problems are strongly coupled (Gardner et al. 1990). For example, farmers often need to collectively invest in infrastructure maintenance (PG) so that enough water (CPR) can be maintained in an irrigation system and used by community members. The question to what extent the coupling of social dilemmas, in particular the contribution to water infrastructure (PG) and restraining from extracting too much water (CPR) affects cooperation is the key contribution of this paper.

In this paper, we analyze how social norms of cooperation with regard to (i) investment in water infrastructure and (ii) water extraction co-evolve with an agent-based model. We observe strong path-dependencies where initial scarcity and poor infrastructure makes the personal sacrifice of cooperating relatively costly. As a result, cooperation erodes, leading to an institutional trap of poor water infrastructure and low cooperation. The opposite can emerge with initial abundance, where cooperation is relatively cheap, and in the long run well-maintained infrastructure, high cooperation, and general prosperity can be observed. Previous research suggests that a system comprising of more conditional cooperators is more likely to be successful in managing common pool resources (Rustagi et al. 2010). This correlation is supported by field work carried out in Cambodia and presented here. However, establishing causality from observational data is a challenge, and our modelling work provides some insights in this regard. While conditional cooperation is typically studied in an experimental or empirical setting, formalizing it in a dynamic framework is not widely considered; but see Richter and Grasman (2013). We formalize conditional cooperation in the model through a reputational mechanism. Individuals have an intrinsic motivation to comply with social norms, and thus deviating from the social norm generates disutility due to psychological costs. Aligning one's behavior with the social norm leads to utility gains (Fehr and Schurtenberger 2018). One of the main reasons for such psychological cost arising from non-compliance with social norms is due to an internal motivation to preserve a positive self-image or reputation in the society (Brekke et al. 2003; 
Fehr and Schurtenberger 2018), which is rooted in the desire to uphold a certain self- or groupidentity (Akerlof and Kranton 2000; Brekke and Howarth 1998). Cooperative behavior is socially desirable and thus leads to higher reputation, while selfishness (so-called defection) is socially undesirable, which thus leads to lower reputation. Reputational considerations can facilitate cooperation among different partners, especially if the social image or reputation of an individual is known within the community (Nowak and Sigmund 1998; Milinski et al. 2002). Thus, at the heart of social norm of conditional cooperation lies the moral motive to align own behavior with social norms at large.

There are a few studies that have analyzed coupled social dilemmas arising from both CPR extracting and investing to a PG before (Botelho et al. 2015; Solstad and Brekke 2011). Solstad and Brekke (2011) model the coupled social dilemmas as a two-stage sequential game, in which income surplus from extracting a CPR in the first stage is used for buying a private good and contributing to a PG in the second stage. They find that the possibility to provide the PG serves as a collective interest and hence can help to overcome the social dilemma in CPR extracting. Their results rest on the assumption that in equilibrium, at least some individuals contribute to the public good. Economic calculus will determine that the marginal (private) benefits equal the marginal (private) costs of providing the public good. Those individuals who will be richer after the first stage will contribute more as the marginal value of money decreases with wealth. This implies that there is no incentive to become richer by not cooperating in the first stage. Botelho et al. (2015) expand the model and test it in a laboratory setting. For both papers, the sequential nature of the social dilemma is salient, and so is the assumption that at least some individuals will have an incentive to contribute. In a natural setting, however, both assumptions may not be met. Also, in reality the benefit structure of water infrastructure, or PG more generally, is often nonlinear and exhibits thresholds, which is what we consider here. The paper starts by presenting the case of water governance in Cambodia and showing some stylized facts from field experiments. In section 3, the agent based model will be presented, before presenting the results in section 4. Finally, section 5 concludes.

\subsection{Conditional cooperation and water governance in Cambodia}

In Cambodia, irrigation is a key element of water governance, as it is salient for small-scale farming, which is very prevalent in the rural areas. Such a system depends largely on collective action of farmers. In many villages, a Farmer Water User Community (FWUC) is present as a 
self-governing institution and plays a main role in regulating water sharing among farmers, as well as collecting contributions to infrastructure maintenance. The success of the FWUC in maintaining a high quality infrastructure to safeguard water availability is mixed. While in some places the water infrastructure is well-functioning, in others the infrastructure is dysfunctional, due to underlying differences in governance and institutional structure (Mak 2017). The mutual feedbacks between individual actions and institutions lead to a complex institutional structure, best described as 'institutional bricolage' (Sakketa 2018), where institutions are the emergent outcome of individual decisions and social interactions. In Ethiopia, field evidence suggests that the presence of conditional cooperators in the system could explain the success in commons forest management (Rustagi et al. 2010). Along the same lines, we hypothesize that the success of user communities to maintain water infrastructure could be linked to conditional cooperation. We explored this in the Kampong Chhnang province of Cambodia, where we run lab-in-thefield experiments with farmers to study conditional cooperation, followed by a survey asking participants to elaborate on their experience with resource scarcity, observed infrastructure quality, and how many users contribute to infrastructure maintenance. The study was reviewed by the Social Sciences Ethic Committee of Wageningen University and registered as a preanalysis plan; see Richter et al. (2020). For more details on the study area, the conditional public goods game, and the complete survey, please see Schuch et al. (2021).

To measure conditional cooperation, we used the same game as Rustagi et al. (2010). In the game, subjects were endowed with 6 bills of $1000 \mathrm{KHR}^{1}$ and were asked to make seven decision rounds on how much to contribute to the public good, knowing what the partner contributes. Using the hierarchical cluster analysis (Fallucchi et al. 2018), the subjects can be classified into five groups: low, medium, and high unconditional cooperators, conditional cooperators, and 'other'; see Schuch et al. (2021) for implementation and experimental procedure. Subjects who are classified into the 'other' behavioral type are those whose contribution scheme does not have a clear pattern. Among these behavioral groups, we are interested in the role of conditional cooperators, who are the ones who try to match the contribution of partners.

Overall, we conducted the games in 21 villages, spread out across three communes. In total, 302 participants played the games (on average, 14 people per village), and 282 participated in the structured survey interviews. Based on the responses, we calculated per village (i) the quality of the irrigation infrastructure, (ii) the contributions to water infrastructure maintenance,

\footnotetext{
${ }^{1}$ Khmer Riel. 4000 KHR is about 1 USD
} 
(iii) experienced water scarcity, and (iv) the share of conditional cooperators. We asked participants to assess the overall quality of the water infrastructure (e.g. canal system and dam) - how well-maintained it is - in their own village on a five-point Likert scale, where 1 means very poor, and 5 is excellent. We then calculated the average score per village. Also, we asked them how much money they paid for getting water for irrigating their rice field in their village. Regarding water scarcity, we asked how many times the household experienced irrigation water scarcity in the past 5 years. We then calculate the average reported number of water scarcity events experienced per village.

\section{Stylized facts from field experiments}

Based on the field experiments, Fig. 1 shows that the presence of conditional cooperators is positively associated with better institutional performance and less water scarcity. First, villages that are composed of more conditional cooperators have better quality of infrastructure (Fig. 1a). Second, villages that comprise a large number of conditional cooperators, have more people reporting to pay for water infrastructure maintenance (Fig. 1b). This suggests that conditional cooperation is positively correlated with institutional outcomes.

(a)

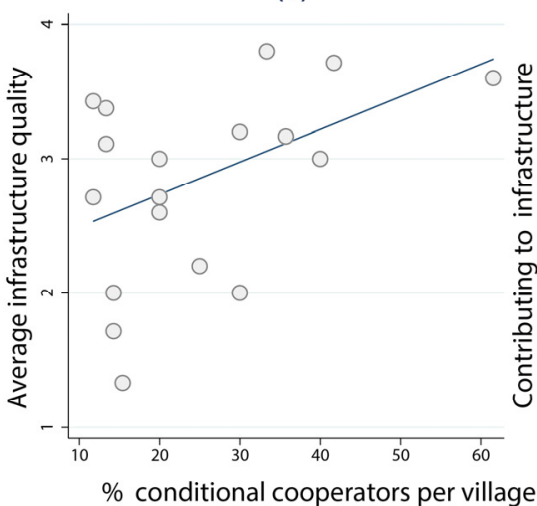

(b)

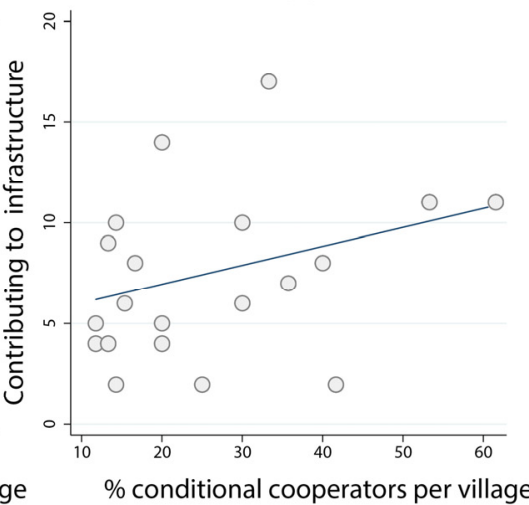

(c)

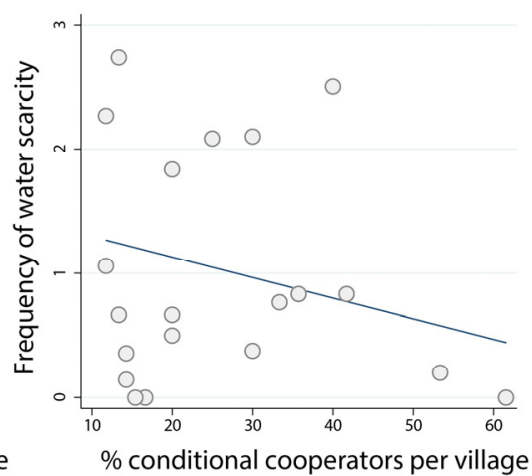

Fig. 1. Conditional cooperation and functioning of institutions across villages in the Kampong Chhnang Province, Cambodia. The institutional performance is measured in terms of (a) infrastructure quality (1 is very poor, and 5 is excellent), (b) number of people paying for water infrastructure, c) frequency of water scarcity.

The results from the field experiments further demonstrate that conditional cooperation positively affects institution in its role to moderate scarcity (Fig. 1c). The larger the number of conditional cooperators in the village, the less water scarcity has been experienced in the village. There are two obvious limitations to these empirical findings. First, while conditional 
cooperation has been measured with an experimental game, the other variables are self-reported and therefore not free of bias and error. Second, we can show correlations, but we do not infer any causality, especially because, the quality of institutions, the willingness to support those institutions, and general cooperativeness all potentially influence each other. Nevertheless, some laboratory and field experiments seem to suggest that players' decisions in the games show some degree of consistency with actual decisions in daily resource uses (Janssen and Anderies 2011). So while our empirical results may not help to disentangle causal channels entirely, they are valuable as they can to inform our modeling work to simulate institutional dynamics 'in silico'.

\subsection{The model}

We consider a community consisting of $N$ agents jointly extracting water as a common-pool resource (CPR), and sharing an irrigation infrastructure as a public good (PG). Water is a common-pool resource (CPR) because a unit of water extracted by an agent is not available to others and everyone has access to the water. Water availability is conditional on the state of the irrigation infrastructure. A well-maintained infrastructure can retain more water than a poorlymaintained one. Keeping the infrastructure well-maintained, however, requires the collective effort of all community members. While it is socially optimal to invest in infrastructure maintenance, doing so is individually costly, tempting self-interested individuals to free ride. After all, one can still benefit from the well-maintained infrastructure even without contributing. Similarly, restraining water extraction is collectively optimal, but requires individual sacrifices. Hence, investing in the PG and extracting from the CPR form social dilemmas. In our model, self-image concerns is the mechanism to uphold conditional cooperation. Each agent faces two types of decision to be made simultaneously: water extraction and investing in infrastructure maintenance. These decisions affect individual utility in two ways. First, there are monetary consequences related to benefits and costs of agricultural practices and infrastructure investments. Second, cooperation has an effect on self-image, where high levels of cooperation gives a positive self-image which translated into a utility gain, while the opposite is true for low cooperation. Cooperation levels are always evaluated against the average behavior in the community, i.e. conditional on social norms. Note that self-image is only one potential interpretation. Our model setup is also consistent with other social mechanisms that encourage cooperative behavior, such as peer pressure, or a loss of reputation. 
Over time, social learning ensures that successful strategies - those that give high utility - are imitated, while those that give low utility are abandoned.

\subsubsection{Investing in water infrastructure}

Agents collectively invest in the infrastructure maintenance. The investment affects water availability, which is shared by all agents in the community. Water availability $(S)$ depends on collective investment $(M)$ and water inflow into the system $(Q)$ and is given by $S=\varepsilon(M) Q$, where $\varepsilon(M)$ is the infrastructure productivity as a function of the collective investment $M$. We define $Q$ as a random variable with expected value $\mu_{Q}$ and standard deviation $\sigma_{Q}$, i.e. $Q \sim N\left(\mu_{Q}, \sigma_{Q}^{2}\right)$. We assume that the infrastructure productivity $\varepsilon(M)$ is a step function, as it requires a minimum level of investment $\mu_{1}$ to be productive and is fully productive when $\mu_{2}$ is provided (see Fig. 2). This stepwise function is also used in a similar context for characterizing the public good (Yu et al. 2015). Hence, the system productivity can be expressed as a function of the collective investment $M(t)$ as

$$
\varepsilon(M)=\left\{\begin{array}{lc}
0 & 0 \leq M(t) \leq \mu_{1} \\
\frac{M(t)-\mu_{1}}{\mu_{2}-\mu_{1}} & \mu_{1}<M(t) \leq \mu_{2} \\
1 & M(t)>\mu_{2}
\end{array}\right.
$$

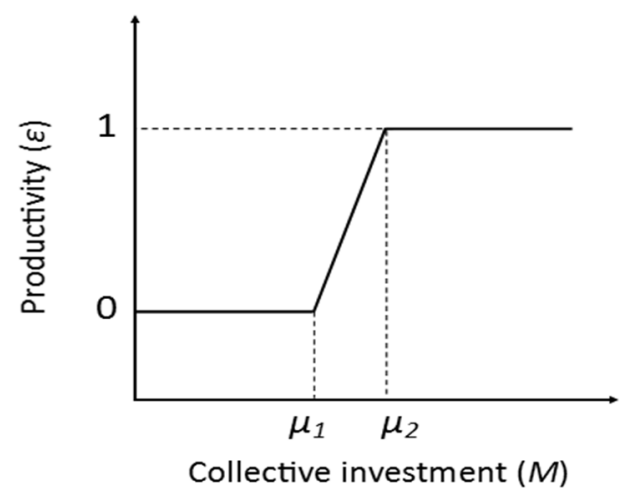

Fig. 2. The relationship between collective investment and system productivity.

\subsubsection{Water extraction}

The second decision involves water extraction. We assume that agents use only two inputs land $l_{i}$ and water $w_{i}$, for the production of a final good $f\left(l_{i}, w_{i}\right)$ which can be sold at price $P$. Land is private and using it incurs a $\operatorname{cost} c_{1}$. Water is a taken from a common pool and extracting it comes at a $\operatorname{cost} c_{2}$ (e.g. pumping cost). The amount of water going to agent $i$ is determined by 
the individual effort $e_{i}$ relative to the aggregate effort $E$ made by all the agents. The amount of water extracted by an agent is thus a fraction of total water availability, i.e. $w_{i}=\left(e_{i} / E\right) S$. Finally, individual investment $m_{i}$ to the water infrastructure comes at $\operatorname{cost} c_{3}$, giving the profit of agent $i$ at time $t$ by

$\pi_{i}(t)=P f\left(l_{i}, w_{i}(t)\right)-c_{1} l_{i}-c_{2} e_{i}(t)-c_{3} m_{i}(t)$

For our model, we assume that agents have fixed land endowment which is exogenously given. We also assume that the production of the final good is represented by a constant elasticity of substitution (CES) function, i.e. $f=\rho\left(w^{\alpha}+l^{\alpha}\right)^{1 / \alpha}$, where $\rho$ is skill factor and $\alpha<1$ measures the degree of complementarity between land and water inputs. For example, when $\alpha \rightarrow 1$, the function exhibits perfect substitutes, meaning it is possible to use either input alone to achieve the same level of output. When $\alpha \rightarrow 0$, the function exhibits some degree of substitution among inputs, i.e. it is possible to use more of one input and less of the other to obtain the same level of output. When $\alpha \rightarrow-\infty$, the function exhibits perfect complementarity among inputs, i.e. the production is possible only if all inputs are used.

\subsubsection{Social dynamics}

We assume that the self-image associated with certain behavior affects individual's utility, depending on how one's actual behavior relates to some normative benchmark what one should do. It is not entirely obvious which normative benchmark is applied in practice, and it may very well be context-dependent (Nyborg 2018). For example, it may be the case that sometimes a Kantian principle is applied based on behavior that would be socially optimal if followed by all, while in other cases social norms based on observed behavior of others may be used as a moral benchmark. We try to reconcile these two different observations. First, we take into account that contribution to a public good and restraining from common pool extraction are perceived as an act of cooperation. Providing more to the public good (and extracting less water) than what the social norm prescribes gives a utility gain. Consequently, providing less to the public good and extracting more water, leads to a utility loss. Second, the size of the utility gain (or loss) is determined how close behavior is to the social norm, i.e. average behavior in the community. This assumption reflects empirical evidence that normative statements about how much one should contribute to a public good, tend to depend on what others do (Hauge 2015). Self-image is thus a function of contributions to maintenance and water extraction and investing efforts. This specification is consistent with the notion of conditional cooperation, as agents are more inclined to cooperate if others do so as well. Formally, we follow Brekke et al. (2003) by 
assuming that self-image is determined by the (squared) deviation from the moral benchmark. Thus, self-image $g_{i}(t)$ of agent $i$ at time $t$ can be expressed as

$g_{i}(t)=\omega_{1} *\left|\bar{e}(t)-e_{i}(t)\right| *\left(\bar{e}(t)-e_{i}(t)\right)+\omega_{2} *\left|m_{i}(t)-\bar{m}(t)\right| *\left(m_{i}(t)-\bar{m}(t)\right)$

where $\bar{e}(t)$ and $\bar{m}(t)$ give the average levels of extraction and investment efforts that can be calculated as $\bar{e}(t)=(1 / N) * \sum_{j \in N} e_{j}(t)$ and $\bar{m}(t)=(1 / N) * \sum_{j \in N} m_{j}(t)$. The parameters $\omega_{1}$ and $\omega_{2}$ can be considered as strength of conditional cooperation or literally as social cohesion. The overall utility of agent $i$ is a function of economic profits $\pi_{i}$ and self-image $g_{i}$, which are imperfect substitutes. Further, we assume that agents may also derive income and self-image from other activities, given by $\hat{\pi}$ and $\hat{g}$. The utility of agent $i$ exhibits Cobb Douglas preferences, which can be written as

$$
u_{i}(t)=\left(\bar{\pi}+\pi_{i}(t)\right)^{\beta}\left(\bar{g}+g_{i}(t)\right)^{1-\beta}
$$

where $\beta$ represent the preference for income compared to self-image.

Fig. 3 shows a conceptual model on the dynamic interplay between individual decisions, water availability, and social dynamics. The decisions of each agent take into consideration the economic gain as well as self-image concerns.

\section{Resource}

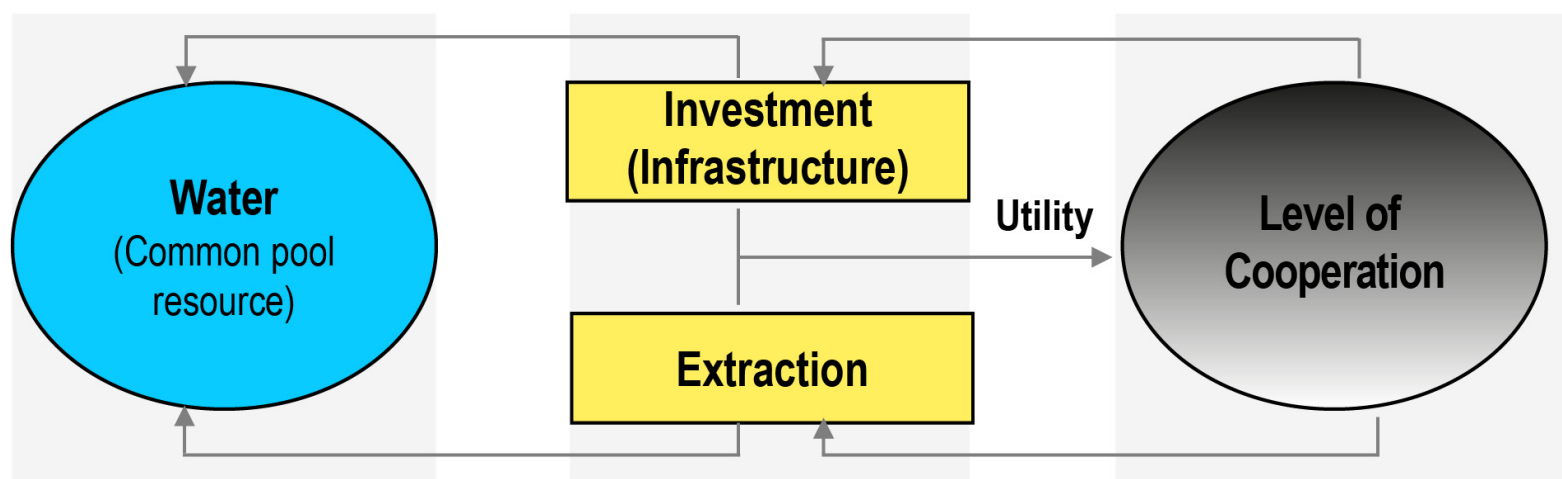

Fig. 3. A conceptual model of the key mechanisms. Agents decide how much to invest in water infrastructure (the public good) and how much water to extract (the common pool resource). The decisions affect water availability, and also profits, self-image and ultimately utility. Social learning guides the social dynamics and the emergent level of cooperation.

\subsubsection{Strategy updating and social learning}

We assume that agents revise their strategy through social learning which is facilitated through observation and imitation of other agents. A focal agent interacts with his four immediate neighbors, occupying the lattice cells in the form of a von Neumann neighborhood given by a $d \times d$ square lattice, where $d$ is the lattice dimension. A fully-occupied lattice thus contains 
$N=d^{2}$ agents. To account for edge effects, agents that are on one side of the borders of the lattice are assumed to be the neighbors of those who are at the opposite side of the border. Each agent considers changing his strategy regarding water extraction and investment to maintenance at time step $t$ by evaluating realized utility in the previous time step. To update his strategies, agent $i$ compares his utility with the utility of his four immediate neighbors. Agent $i$ identifies the strategy of the neighbor with the highest utility, and imitates it with a probability $p$, which is an increasing function of the utility difference $u_{j}(t)-u_{i}(t)$. We use a logistic function to model the imitation probability as follows

$p\left[\left(e_{i} \rightarrow e_{j}\right) \&\left(m_{i} \rightarrow m_{j}\right)\right]=\frac{1}{1+\exp \left(-\lambda\left(u_{j}-u_{i}\right)\right)}$

where $\lambda \geq 0$ can be considered as imitation strength which measures how strongly the utility difference influences the decision of an agent to switch to a better-off strategy. $\lambda \rightarrow 0$ or $u_{j}=$ $u_{i}$ means agent $i$ tosses the coin to decide if he imitates the strategy of agent $j . \lambda \rightarrow+\infty$ corresponds to high imitation strength, meaning that a successful strategy is always imitated. Consequently, a small $\lambda$ depicts low imitation strength, meaning that a successful strategy is less likely imitated. When updating the strategy, we also consider stochastic errors (mean of zero and standard deviation of 0.01) when imitating extraction and investment levels, resulting in a strategy that is a bit lower or higher than the copied strategy. 
Table 1. Model variables and parameters at default values

\begin{tabular}{lll}
\hline \hline Symbols & Definition & Value \\
\hline \hline Variables & & \\
$e_{i}$ & Individual extracting effort & \\
$m_{i}$ & Individual investment effort & \\
Parameters & & \\
$N$ & Community size & 400 \\
$t$ & Simulation length & 200 \\
$\bar{Q}$ & Mean resource inflow & 100 \\
$\sigma_{Q}$ & Standard deviation of resource inflow & 20 \\
$P$ & Sale price & 1 \\
$l$ & Land size & 1 \\
$c_{1}$ & Unit cost of farming per unit land & 0.5 \\
$c_{2}$ & Unit cost of extraction effort & 0.5 \\
$c_{3}$ & Unit cost of investment effort & 0.5 \\
$\omega_{1}, \omega_{2}$ & Social pressure in relation to extraction and investment respectively & 2 \\
$\alpha$ & Degree of complementarity & 0.5 \\
$\bar{g}$ & Exogenous reputation & 0.5 \\
$\bar{\pi}$ & Exogenous profit & 0.5 \\
$\mu_{1}$ & Minimum investment threshold & 80 \\
$\mu_{2}$ & Optimum investment threshold & 320 \\
$\lambda$ & Imitation strength & 1 \\
\hline & &
\end{tabular}

\subsection{Results}

First we explore the temporal evolution of extraction and investment efforts and the general dynamics of the system. In the model, the evolution of the state variables is path-dependent, giving rise to alternative stable states depending on initial conditions. Thus, we first explore under which conditions the good equilibrium of institutional prosperity emerges. Second, we turn to the question under which conditions an institutional trap may occur. Third, we analyze the model behavior without the conditional cooperation mechanism. Fourth, we analyze to escape the institutional trap and what is needed to reach the 'good' equilibrium of institutional prosperity. Finally, we investigate the sensitivity of key parameters with regard to flipping between the alternative stable states. 


\subsubsection{The good equilibrium: Institutional prosperity}

We simulated the model with default parameter values given in Table 1. The distributions of the investment and extraction efforts at the initial time step were modelled following a bimodal distribution or generally referred to as "Gaussian mixture distribution" to reflect the presence of different groups in the community, rather than deviation around one established norm. We also simulated our model with a normal distribution and generally found similar results. We are interested to see if a society that is largely comprised of cooperative individuals initially may remain in such desirable condition over time. In our model, the condition is mimicked by having at the initial time step a large fraction of agents (80\%) having high investment effort (mean investment effort of 0.8 and variance of 0.01$)$, and only a small fraction of them (20\%) exerting low investment (mean investment effort of 0.2 and variance of 0.01 ). Likewise, $80 \%$ of the agents have low extraction effort (mean extraction effort of 0.2 and variance of 0.01 ) and 20\% have high extraction effort (mean extraction effort of 0.8 and variance of 0.01 ). Each model was run 50 times to account for stochasticity. The stochastic components of the model are mainly due to different investment and extraction efforts at the initial time step, and the strategy updating.

Over time the model reaches an equilibrium state in which agents exert relatively high investment levels (average of around 0.7) (Fig. 4a), which is slightly lower than the starting value around 0.8 for most individuals. Those high investment levels support relatively high extraction effort (Fig. 4b) (average around 0.9), which is substantially higher than the extraction at initialization $(80 \%$ had an extraction level around 0.2$)$. Due to stochasticity, the system is out of equilibrium for some simulation runs, sometimes over prolonged periods of time (the light shading in Fig. $4 \mathrm{a} \&$ b). Since the aggregate investment effort made by all agents is directly related to system productivity (Equation (1)), higher investment effort means higher system productivity (Fig. 4c), which also leads to high profits (Fig. 4d). 
(a)

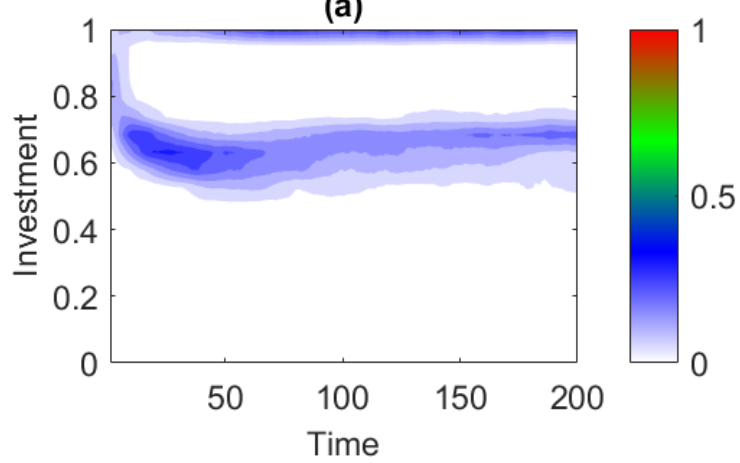

(c)

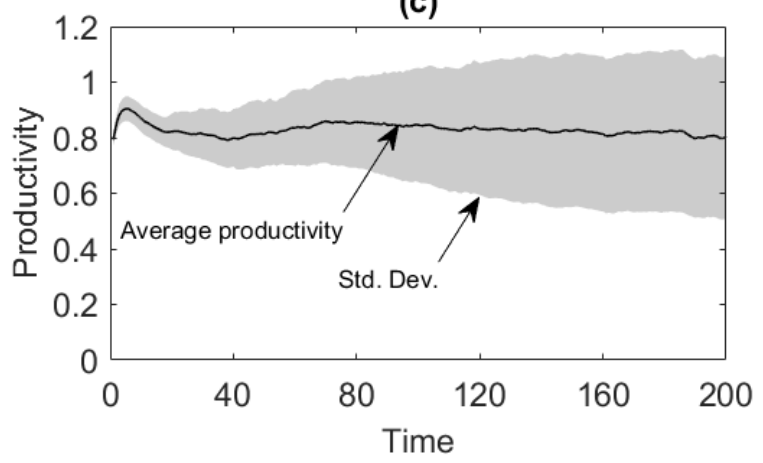

(b)

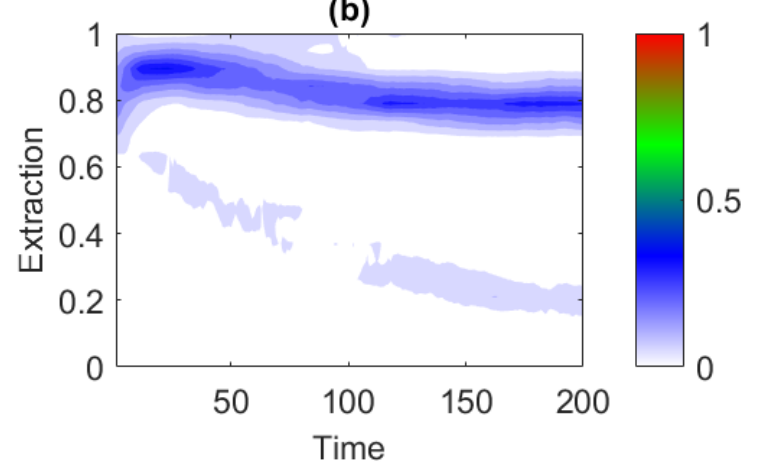

(d)

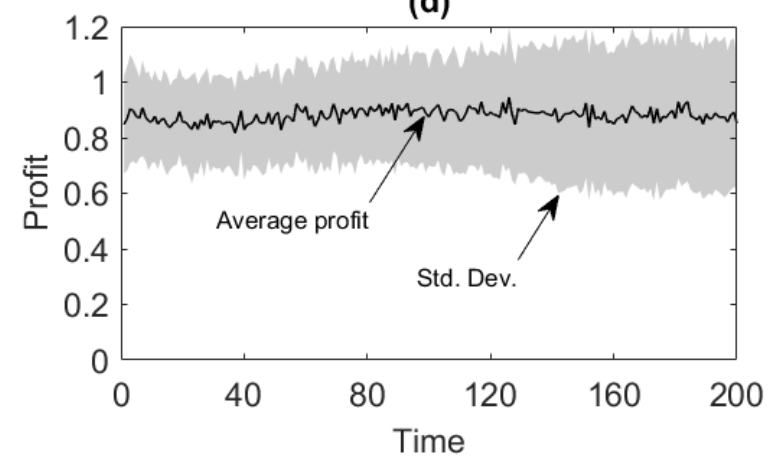

Fig. 4. Temporal evolution of extraction and investment efforts when the public good is initially in a good condition $-80 \%$ of high-contribution agents and $20 \%$ of high-extraction agents at $t=0$. X-axis stands for simulation time. Y-axis shows (a) investment level, (b) extraction level, (c) system productivity, and (d) profit. Colorbar represents the relative frequency of extraction and investment efforts among all agents at each time step over 50 repeated runs.

\subsubsection{The bad equilibrium: Institutional trap}

In this scenario, we look into a condition in which the society is largely comprised of selfinterested individuals, i.e. those who are not willing to invest to the public goods but continue to extract the common-pool resource at a high rate. We are interested to see if the society that is initially in such a bad condition, would become more cooperative over time or remain trapped in this condition. In the 'bad equilibrium', at the initial time step $20 \%$ of the agents make high investment effort (mean investment effort of 0.8 ), while $80 \%$ of the agents have low investment effort (mean investment effort of 0.2). Also, a large number of agents (80\%) exert high extraction effort (mean extraction effort of 0.8 ) and 20\% low extraction effort (mean extraction of 0.2$)$.

Fig. 5 shows that over time the investment effort further erodes and eventually no investments will be made (Fig. 5a). As a result, the system gets trapped in a very low-productivity state in which resource availability is close to zero (Fig. 5c). Consequently, most agents also lower their extracting effort over time (Fig. 5b), simply because it is not economically viable. Overall, 
profits are much lower than in the 'good equilibrium' and only some exogenous profits remain (Fig. 5d).

(a)

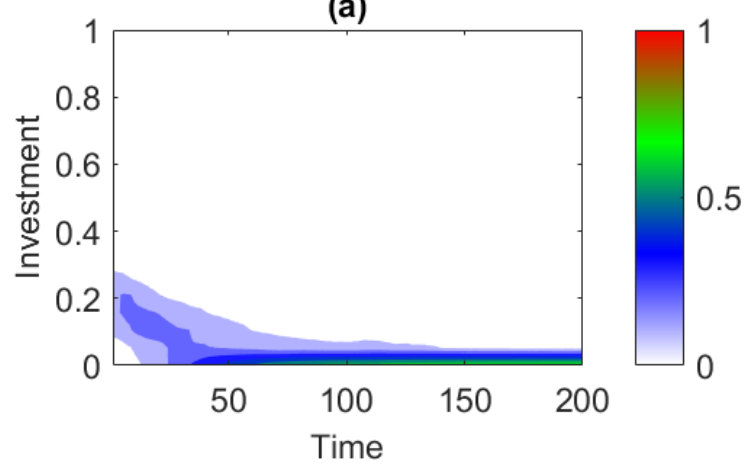

(c)

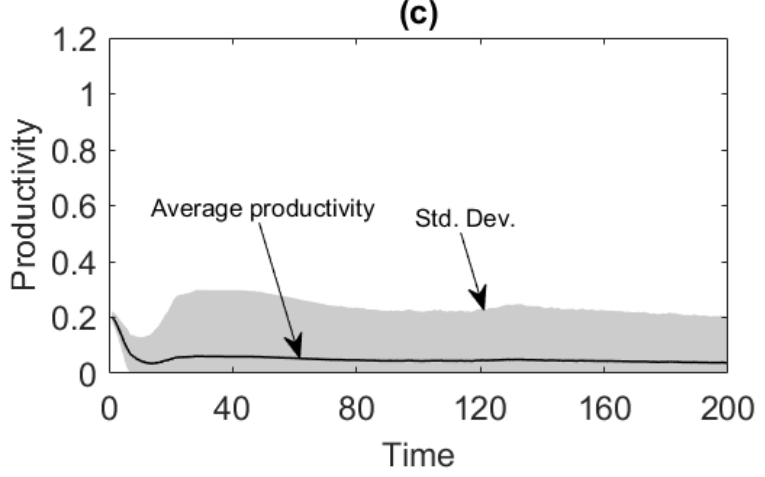

(b)

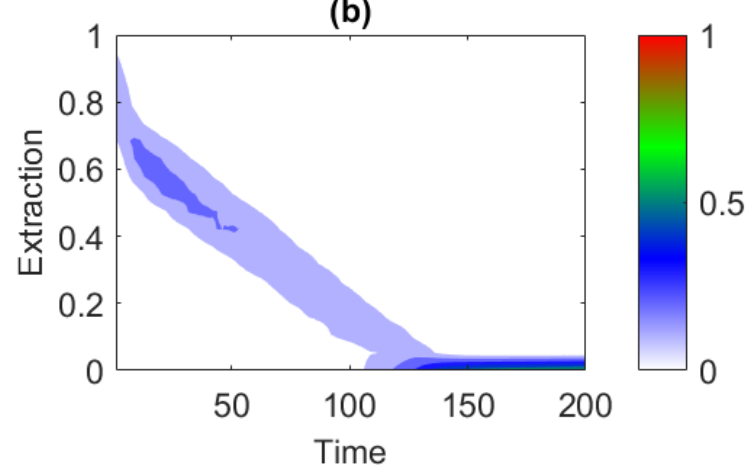

(d)

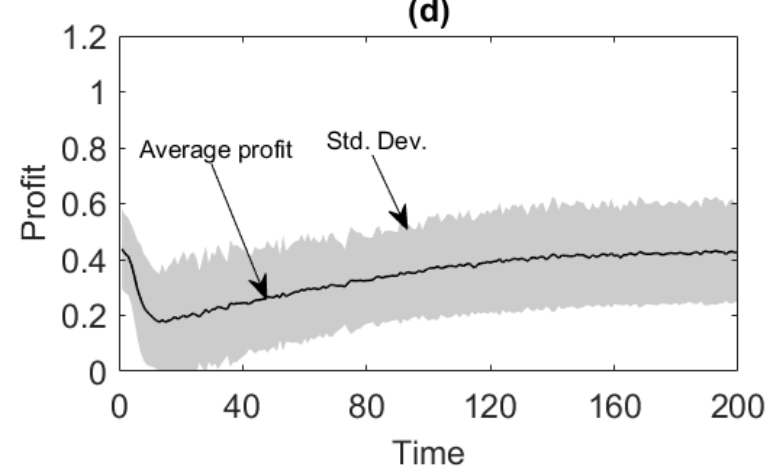

Fig. 5. Temporal evolution of extraction and investment efforts when the public good is initially in a bad condition-20\% high-contribution agents and $80 \%$ high-extraction agents at $t=0$. X-axis stands for simulation time. Y-axis stands for (a) investment level, (b) extraction level, (c) system productivity, and (d) profit. Colorbar represents the relative frequency of extraction and investment efforts among all agents at each time step over 50 repeated runs.

\subsubsection{In the absence of self-image considerations, cooperation collapses}

Our model entails a coupled social dilemma in CPR extraction and PG investment, where conditional cooperation via self-image considerations mitigates freeriding. Each agent's utility, as determined in Equation (4), is the result of economic and self-image outcomes. To consider a case in which conditional cooperation is absent, the coefficients $\omega_{1}$ and $\omega_{2}$ were set to zero. The self-image $g_{i}$ of each agent, as depicted in Equation (3), thus becomes zero, meaning that only economic considerations remain in the utility function.

Fig. 6 illustrate the case where self-image concerns are absent. As expected, in this case cooperation collapses. The public good investment becomes largely under-provided (Fig. 6a). As a result, system productivity collapse (Fig. 6c). Under this condition any extraction effort 
exerted by agents is not economically viable, and extraction declines over time (Fig. 6b). Some level of exogenous profit, though, remain (Fig. 6d).

(a)

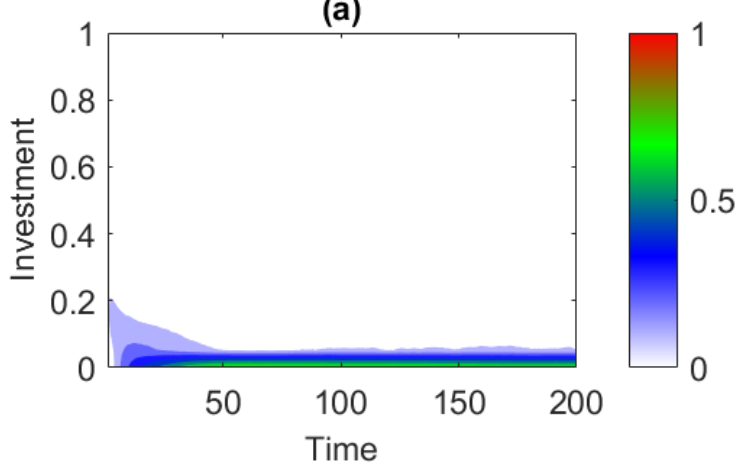

(c)

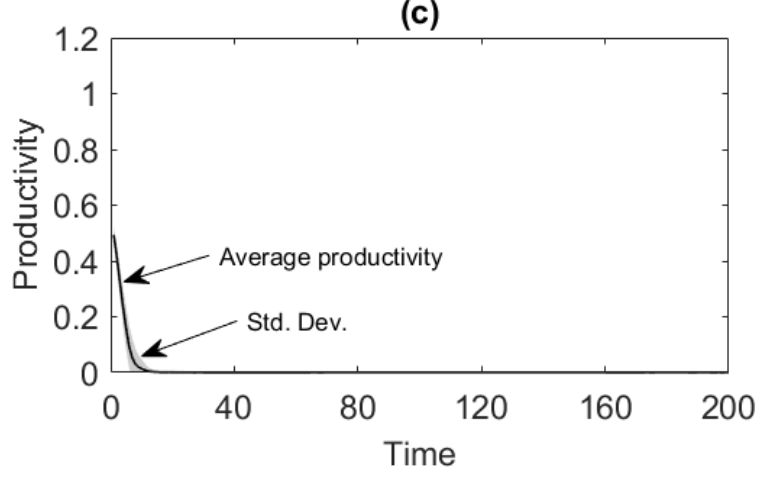

(b)

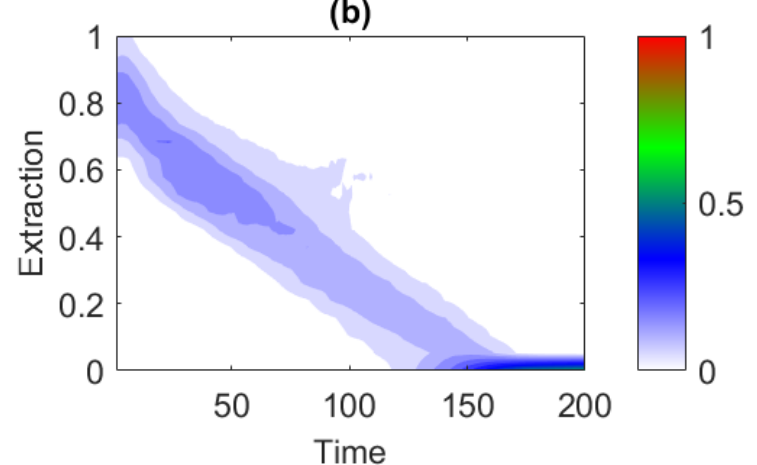

(d)

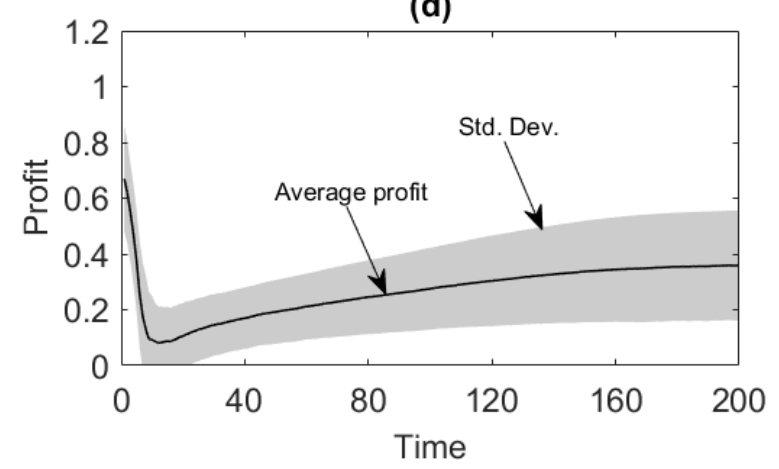

Fig. 6. Temporal evolution of extraction and investment efforts, and corresponding outcomes when the reputational mechanism is absent $\left(\omega_{1}=0.0, \omega_{2}=0.0\right)$. All other parameters are kept at default values. $\mathrm{X}$-axis stands for simulation time. Y-axis stands for (a) investment level, (b) extraction level, (c) system productivity, and (d) profit. Colorbar represents the relative frequency of extraction and investment efforts among all agents at each time step over 50 repeated runs.

\subsubsection{Social cohesion to escape the institutional trap}

We have shown earlier that if the system initially consists largely of low contributing and high extracting agents, the system remains trapped in the bad equilibrium (Fig. 5). This condition can also be considered as resource scarcity condition, since the public good is largely underprovided, leading to less resource available in the system. Fig. 7 analyzes the case where the strength of conditional cooperation is high, i.e. $\omega_{1}=3$ and $\omega_{2}=3$. A high strength of conditional cooperation means agents have high tendency to align own extraction and investment decisions with what the social norm describes. Further, high strength of conditional cooperation implies strong social cohesion (Röttgers 2016). We find that if conditional cooperation is stronger, the institutional trap can be escaped and the investments are high (Fig. 
7a). As a result, system productivity is high (Fig. 7c), more resources are available, and high extraction is supported (Fig. 7b). Consequently, average profits are also high (Fig. 7d).

(a)

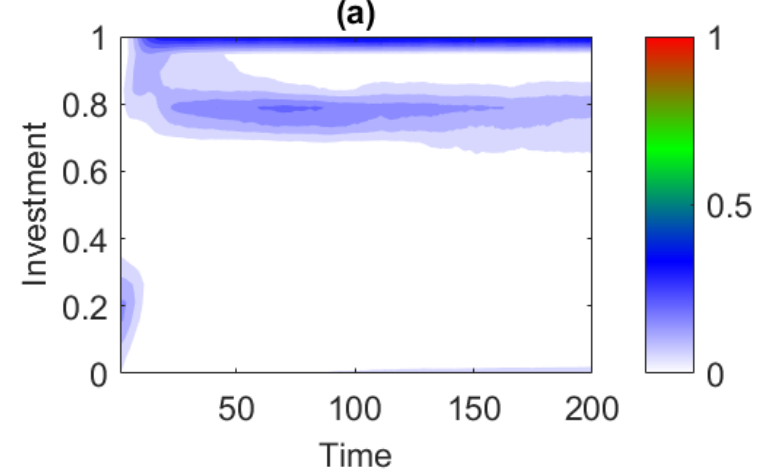

(c)

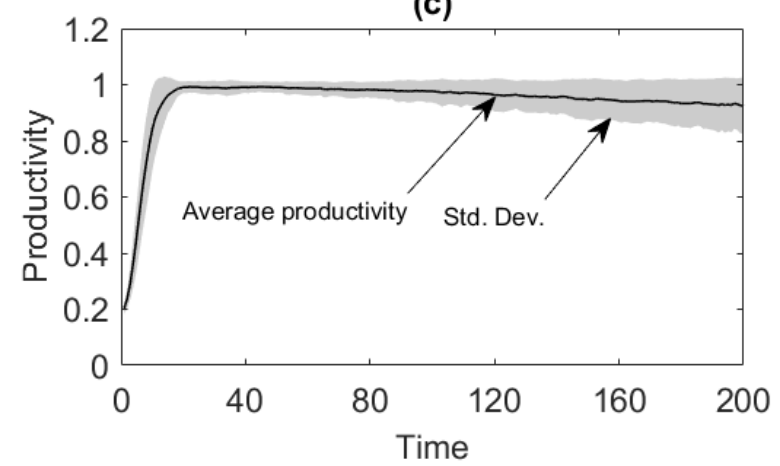

(b)

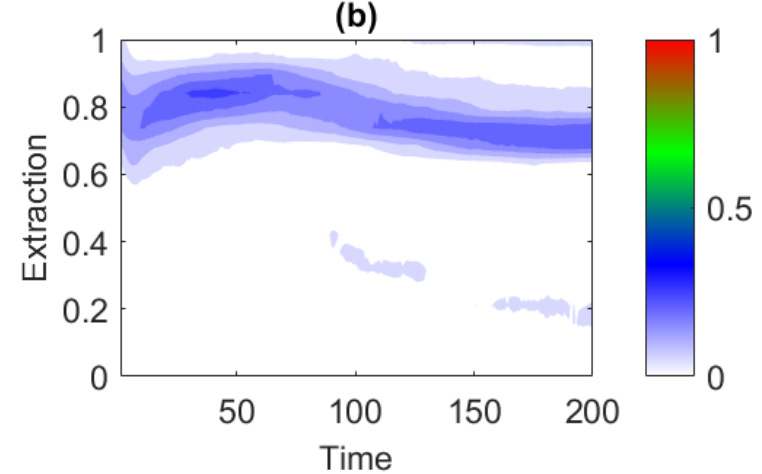

(d)

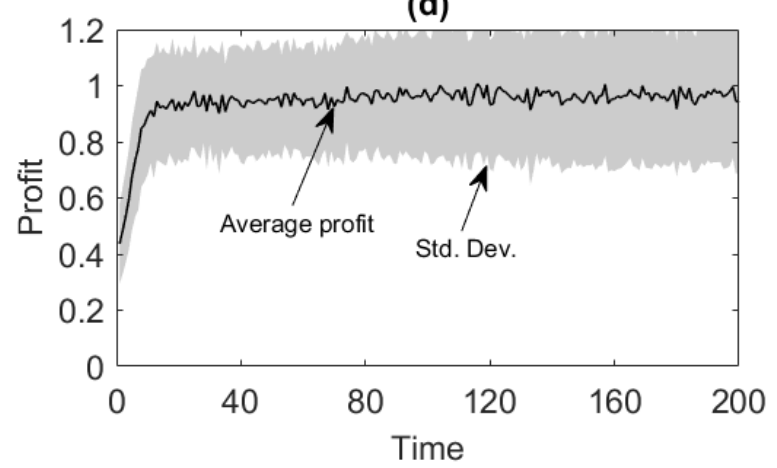

Fig. 7. Temporal evolution of extraction and investment efforts when the public good condition is initially bad ( $80 \%$ of low contributing agents and $80 \%$ of high-extraction agents at $t=0)$, but in the case where the strength of conditional cooperation is high $\left(\omega_{1}=3.0, \omega_{2}=3.0\right)$. All other parameter values are kept at default values. X-axis stands for simulation time. Y-axis stands for (a) investment level, (b) extraction level, (c) system productivity, and (d) profit. Colorbar represents the relative frequency of extraction and investment efforts among all agents at each time step over 50 repeated runs.

\subsubsection{What triggers alternative stable states?}

We run a series of simulations to study effects of key parameters of the model on extraction and investment efforts of agents (Fig. 8). First, we consider the role of economic costs As cost per unit of land $\left(c_{1}\right)$ increases, extraction effort slightly increases and investment goes down. There is a tipping point, above investment collapses, and so do productivity and extraction (Fig. $8 \mathrm{a} \& \mathrm{~b})$. When cost of extraction effort $\left(c_{2}\right)$ goes up, agents lower the extraction effort but at the same time they also lower the investment effort (Fig. 8e \& f). Again, a tipping point can be observed at a critical parameter value for $c_{2}$. When the cost of investment in infrastructure $\left(c_{3}\right)$ increases, investment goes down and extraction goes up (Fig. $8 \mathrm{i} \& \mathrm{j}$ ). Beyond a critical level of $c_{3}$ the system collapses. After having analyzed the role of costs, let us now turn to social 
pressure. Higher social pressure in relation to extraction $\left(\omega_{1}\right)$ - meaning that it is socially costly to deviate one's extraction effort from the social norm - leads to lower extraction, but investment remains unchanged (Fig. $8 \mathrm{c} \&$ d). Higher social pressure in relation to investment $\left(\omega_{2}\right)$ leads to both higher extraction and investment since more aggregate investment means more resource is available to be extracted (Fig. $8 \mathrm{~g} \& \mathrm{~h}$ ). Again, alternative stable states can be observed. Finally, higher resource inflow leads to higher extraction and investment efforts, giving rise to alternative stable states (Fig. $8 \mathrm{k} \& 1$ ).

(a)

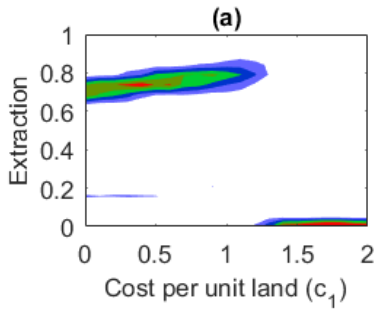

(b)

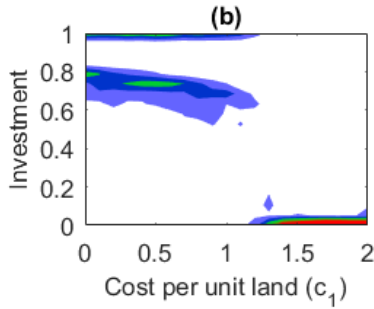

(c)

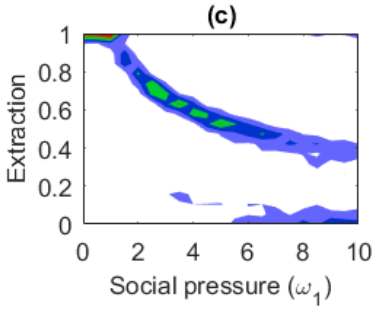

(d)

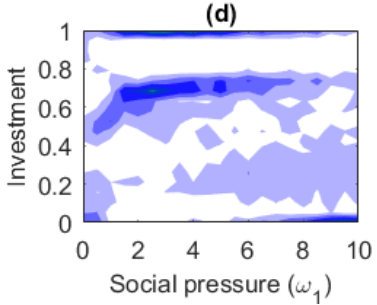

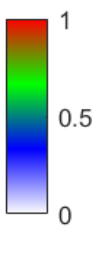
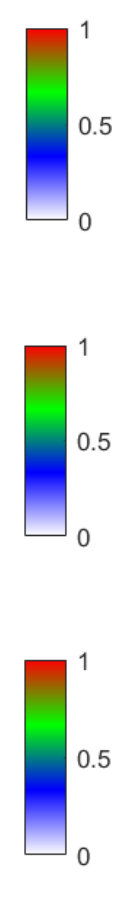

(e)

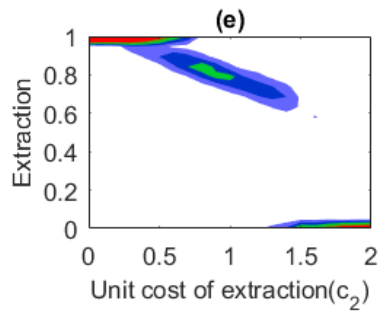

(f)
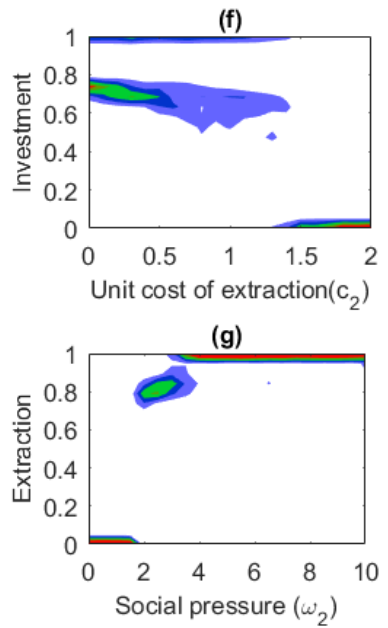

(h)

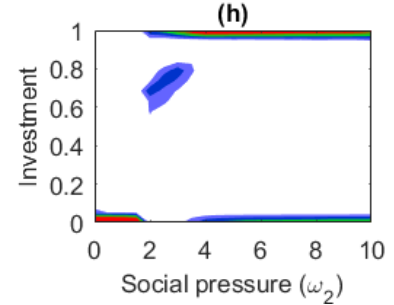

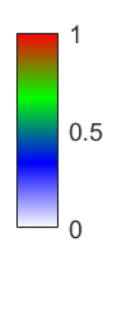
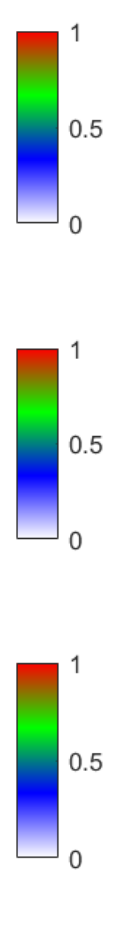

(i)
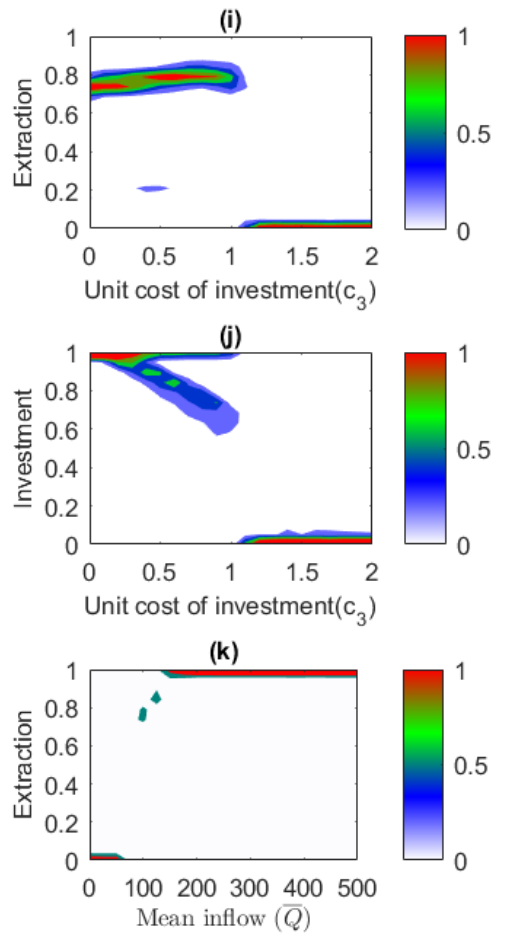

(I)
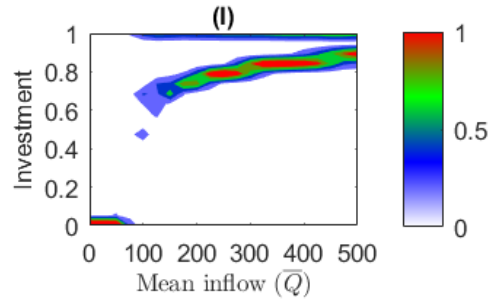

Fig.8. One-at-a-time sensitivity plot of key model parameters against investment and extraction effort. To account for stochasticity, we run the model 50 times repeatedly for each single value of the parameters.

\subsection{Discussions and Conclusion}

We have developed an agent-based model, in which agents have to decide how much effort to contribute to a common water infrastructure and how much water to extract. This decision is based on economic considerations, as well as reputational concerns, where the own decision is 
evaluated against the social norm. We find that the system features alternative stable states, depending on initial conditions. If the system has a functioning water system initially and a high level of cooperation, prosperity can be created, which facilitates further investments in water infrastructure, fostering cooperation further. If the system features initial scarcity, cooperation is relatively costly, further undermining investments in water infrastructure, potentially leading to an institutional trap. If the system is comprised of more conditional cooperators, however, the initial scarcity condition can be mitigated. These findings are well in line with the stylized facts observed in the field. Our field experiments in Cambodia suggest that conditional cooperation is positively correlated with better institutional outcomes, and in addition, can mitigate scarcity conditions.

The results from the model establish that without reputational mechanisms - driven by conditional cooperation - self-governance will collapse (Fig. 6), and that even if the mechanism is in place, cooperation may not evolve and the system may be trapped in a situation of severe scarcity (Fig. 5). Stronger social capital can, however, facilitate an escape of the trap and facilitate cooperation under such scarcity condition (Fig. 7). In line with the field evidence the modeling results confirm that conditional cooperation could be beneficial for institutions to prosper by further facilitating cooperation under scarcity.

In the context of coupled social dilemmas arising from contributing to the public good and extracting of a common pool resource, previous studies establish that if the public good is provided, cooperative resource extracting can be achieved (Botelho et al. 2015; Solstad and Brekke 2011). An open question is why the public good is provided by some agents in the first place. We offer an explanation by formalizing the notion of conditional cooperation. Inspired by the work of Rustagi et al. (2010), who show that communities consisting of more conditional cooperators are more successful in governing the forest commons, we explore this for the case of local water governance in Cambodia, using lab-in-the-field experiments. Our model results support the idea that conditional cooperation can mitigate initial scarcity and further facilitate the contribution to the public good and hence overall cooperation. Further, we show in our model that if the community is largely comprised of low contributing agents, the system remains trapped in the regime of low contribution to the public good and thus remains trapped with poor water infrastructure. This is especially the case if agents are heterogenous in contribution and extraction levels. Our modelling results are in line with experimental evidence, suggesting that the community that is comprised of more conditional cooperators is more 
successful in irrigation water governance, and one of the reason for this is the scarcitymoderating effect of conditional cooperators.

Our study has two implications for researchers and practitioners. First, when evaluating the impacts of drivers that may bring about scarcity (e.g. climate change) it is important to consider that the institutional system may respond to scarcity, potentially aggravating or moderating scarcity. We have shown here that social capital in general, and conditional cooperation in particular are important mechanisms to be considered. Second, our study cautions against considering coupled social dilemmas, such as common pool resources and public goods problems in isolation, as the unfolding dynamics may be very different and could have significant implications for the long-term sustainability of social-ecological systems. 


\section{References}

Akerlof, George A., and Rachel E. Kranton. 2000. "Econmics and Identity." Quarterly Journal of Economics CXV (3): 715-53.

Botelho, Anabela, Ariel Dinar, Lígia M.Costa Pinto, and Amnon Rapoport. 2015. "Promoting Cooperation in Resource Dilemmas: Theoretical Predictions and Experimental Evidence." Journal of Behavioral and Experimental Economics 54: 40-49. https://doi.org/10.1016/j.socec.2014.11.007.

Brekke, Kjell Arne, and Richard B Howarth. 1998. "The Social Contingency of Wants: Implications for Growth and the Environment," no. 227: 22.

Brekke, Kjell Arne, Snorre Kverndokk, and Karine Nyborg. 2003. “An Economic Model of Moral Motivation." Journal of Public Economics 87 (9-10): 1967-83. https://doi.org/10.1016/S0047-2727(01)00222-5.

Fallucchi, Francesco, R. Andrew Luccasen, and Theodore L. Turocy. 2018. "Identifying Discrete Behavioural Types: A Re-Analysis of Public Goods Game Contributions by Hierarchical Clustering.” Journal of the Economic Science Association 5 (2): 238-54. https://doi.org/10.1007/s40881-018-0060-7.

Fehr, Ernst, and Ivo Schurtenberger. 2018. "Normative Foundations of Human Cooperation Review-Article." Nature Human Behaviour 2 (7): 458-68. https://doi.org/10.1038/s41562-018-0385-5.

Gardner, Roy, Elinor Ostrom, and James M. Walker. 1990. “The Nature of Common-Pool Resource Problems." Rationality and Society 2 (3): 335-58.

Hauge, Karen Evelyn. 2015. "Moral Opinions Are Conditional on the Behavior of Others." Review of Social Economy 73 (2): 154-75. https://doi.org/10.1080/00346764.2015.1035910.

Janssen, Marco A., and John M. Anderies. 2011. "Governing the Commons: Learning from Field and Laboratory Experiments.” Ecological Economics 70 (9): 1569-70. https://doi.org/10.1016/j.ecolecon.2011.03.023.

Lansing, J. Stephen, Stefan Thurner, Ning Ning Chung, Aurelie Coudurier-curveur, Cagil Karakas, Kurt A. Fesenmyer, and Lock Yue Chew. 2017. "Adaptive Self-Organization of Bali's Ancient Rice Terraces." Proceedings of the National Academy of Sciences 114 (25): 6504-6509. https://doi.org/10.1073/pnas.1605369114.

Mak, Sithirith. 2017. "Water Governance in Cambodia: From Centralized Water Governance 
to Farmer Water User Community." Resources 6 (3): 44.

Milinski, Manfred, Dirk Semmann, and Hans Jürgen Krambeck. 2002. "Reputation Helps

Solve the "Tragedy of the Commons." Nature 415 (6870): 424-26.

https://doi.org/10.1038/415424a.

Nowak, Martin A., and Karl Sigmund. 1998. "Evolution of Indirect Reciprocity by Image

Scoring." Nature 393 (6685): 573-77. https://doi.org/10.1038/31225.

Nyborg, Karine. 2018. "Social Norms and the Environment." Annual Review OfResource

Economics 10: 405-23. https://doi.org/https://doi.org/10.1146/annurev-resource100517-023232.

Ostrom, Elinor. 1990. Governing the Commons: The Evolution of Institutions for Collective Action. Cambridge University Press.

Richter, A., and Johan Grasman. 2013. "The Transmission of Sustainable Harvesting Norms When Agents Are Conditionally Cooperative.” Ecological Economics 93: 202-9.

Richter, A., E. Schuch, and T. Nhim. 2020. “Cooperation under Uncertainty in Natural Resource Management.” https://doi.org/10.17605/OSF.IO/U3GC8.

Röttgers, Dirk. 2016. “Conditional Cooperation, Context and Why Strong Rules Work - A Namibian Common-Pool Resource Experiment.” Ecological Economics 129: 21-31. https://doi.org/10.1016/j.ecolecon.2016.03.013.

Rustagi, Devesh, Engel Stefanie, and Michael Kosfeld. 2010. "Conditional Cooperation and Costly Monitoring Explain Success in Forest Commons Management." Science 330 (6006): 961-65. https://doi.org/10.1126/science.1193649.

Sakketa, Tekalign Gutu. 2018. "Institutional Bricolage as a New Perspective to Analyse Institutions of Communal Irrigation: Implications towards Meeting the Water Needs of the Poor Communities." World Development Perspectives 9 (January 2017): 1-11. https://doi.org/10.1016/j.wdp.2017.11.003.

Schuch, Esther, Simone Dirks, Tum Nhim, and Andries Richter. 2021. "Cooperation under Social and Strategic Uncertainty - The Role of Risk and Social Capital in Rural Cambodia." Journal of Behavioral and Experimental Economics 90 (May 2020): 101642. https://doi.org/10.1016/j.socec.2020.101642.

Solstad, Jan Tore, and Kjell Arne Brekke. 2011. "Does the Existence of a Public Good Enhance Cooperation among Users of Common-Pool Resources?” Land Economics 87 (2): 335-45. https://doi.org/10.1353/lde.2011.0023.

Yu, David J, Murad R Qubbaj, Rachata Muneepeerakul, John M Anderies, and Rimjhim M Aggarwal. 2015. "Effect of Infrastructure Design on Commons Dilemmas in Social- 
Chapter 3

Ecological System Dynamics." Proceedings of the National Academy of Sciences of the United States of America 112 (43): 1410688112-.

https://doi.org/10.1073/pnas.1410688112. 


\section{Authors' contribution}

T.N. and A.R. designed the research. TN developed the model and carried out the analysis. All authors contributed to writing the final manuscript.

\section{Acknowledgments}

T.N. gratefully acknowledges funding from the European Commission through the Alfabet project under the EU Erasmus Mundus Program. 



\title{
Chapter 4-Multi-level water self-governance-cooperation within and between communities in Cambodia
}

\author{
Tum Nhim
}

\begin{abstract}
Managing shared resources remains a challenge, and highly relevant in the context of common pool resources (CPR) or public goods (PG). Restraining CPR extraction or investing to a PG is collectively beneficial, , but costly for individuals, giving rise to social dilemmas that may lead to inefficient use or depletion of the resource. This study employs a stylized evolutionary agentbased model to analyse under what conditions cooperation emerges and spreads between and within two communities that share water as a CPR and infrastructure as a PG. The upstream community gets the first claim of water, while the downstream gets what remains. The upstream, however, depends on investment in water infrastructure from the downstream for the provision of the CPR. Agents decide on their extraction and investment levels over time, starting from heterogeneous and random strategies. While extracting more water or investing less in infrastructure makes an agent economically better off, doing so erodes his self-image which can potentially inflict loss on his economic performance. Agents thus make decisions balancing doing well economically and doing good morally by learning from their best performing neighbours and from random encounters with members from the other community. The results show that the reputation mechanism is salient to maintain cooperation. Further, the model results show that increased cooperation in the upstream community leads to more cooperation in the downstream community as well as higher welfare overall, but only if there are social interactions between communities. Overall, this study highlights the tension to cooperate within and between communities of resource users, providing theoretical understanding for sustainable governance of common pool resources and public goods at multiple scales.
\end{abstract}

Key words: Multi-level cooperation, coupled social dilemmas, common-pool resources, public goods, agent-based modelling 


\subsection{Introduction}

Environmental degradation, overexploitation of natural resources, and greenhouse gas emissions are major concerns for human society (Rockström et al. 2009; Dietz et al. 2003). None of these problems are private, and have two characteristics in common: they involve multiple individuals and groups, and collective action at multiple scales is needed to solve these problems (Pahl-Wostl 2009; Walker et al. 2009). The failure of collective action is evident at various scales. At the global scale, one of the most challenging issues for humanity is climate change. While individuals and nations are collectively better off if all cooperate by reducing greenhouse gas emissions, each is tempted to defect and freeride on effort of others since everyone can still benefit from the reduction of greenhouse gas emissions, even if they do not contribute any effort. This is a public good (PG) dilemma. At a regional scale, transboundary water sharing is another example illustrating the challenge for collective action. Globally, human's water withdrawal in the upstream of a river basin can potentially exacerbate water scarcity in the downstream area (Veldkamp et al. 2017). This problem entails a suboptimal or inefficient use of water among upstream and downstream users, due to a lack of coordination and cooperation, and is a consequence of a social dilemma situation in which upstream users overuse water for personal gains since the benefit of doing so is private, while the costs are shared by all upstream and downstream users. This is a common pool resource dilemma. In many real-world situations PG and CPR problems are strongly coupled and manifest at multiple scales (Gardner et al. 1990; Ostrom 2010; 2009). An example is famers collectively investing in infrastructure maintenance (PG) so that enough amount of water (CPR) can be maintained in an irrigation system and shared by upstream and downstream communities.

Whether cooperation can be maintained when resource users in a single community face both CPR and PG dilemmas has been analysed in (Nhim and Richter 2021). A few other studies analyse coupled social dilemmas arising from both CPR harvesting and investing to a PG (Botelho et al. 2015; Solstad and Brekke 2011). They have shown that cooperation can be preserved when the PG is present, provided that a certain number of agents contribute to the public good. The presence of a PG may create economic interdependencies where cooperation in CPR harvesting is conditional upon cooperation in PG provisioning. For instance, failing to collect enough fee for infrastructure maintenance (cooperation in PG) may result in critical water shortage which then undermines cooperation in water harvesting (cooperation in CPR). 
A wealth of studies have shown that cooperation in CPR or PG can be sustained under informal arrangement mechanisms, especially costly peer enforcement (Sethi and Somanathan 1996) or intrinsic social disapproval (Tavoni et al. 2012). While these mechanisms are shown to be effective in a small community where frequency of encounters among members is high, whether it works in a large community or between communities remains unclear. Typically, in a large community encounters among members are rare and thus peer enforcement or social disapproval might be ineffective or very costly. When it comes to facilitating cooperation among different communities or in a large group, informal mechanism such as indirect reciprocity based on reputation is shown to be working well under certain conditions (Nowak 2006). Basically, the idea is that direct encounters among agents are not necessary and that an agent acting cooperatively is tagged as having good image which then is more likely to be rewarded later on by others (Nowak and Sigmund 1998). However, how this simple mechanism works in practice is not obvious, for example, how the image is evaluated and assigned to a specific individual. Also, memory of agents regarding the behaviour of others might be lost over time. Another mechanism considered is the reputation driven by moral motivation or a desire to fit in a social group (Brekke et al. 2003). In this mechanism, an agent feels morally obliged to always keep an eye on his own decision, trying to align his behaviour with the behaviour that is considered socially morally acceptable, for example average behaviour in the group.

Whereas enforcement mechanisms such as peer punishment (Sethi and Somanathan 1996), reputation based on image scoring (Nowak and Sigmund 1998), and reputation driven by moral motivation (Brekke et al. 2003) may help sustain cooperation in small or large communities under certain conditions, we know surprisingly little about other mechanisms for intergroup cooperation (Robinson and Barker 2017). The nature of interactions between groups can potentially affect cooperation. Further, when the dynamics of group interactions are studied, they are typically analysed in a public goods environment (Perc et al. 2013; Wang et al. 2013). How cooperation emerges and spread across communities who face a combined social dilemmas of common-pool resource extraction and public goods investment, however, remain less understood, and is the focus of this paper. 


\subsection{Objectives and Contributions}

The objective of this research is to understand how cooperation evolves and spreads between individuals and communities when the interaction between a common pool resource and a public good is present. In particular, I aim to understand (i) whether cooperation in one community lead to more or less cooperation in the other; and (ii) how interactions between communities affect cooperation in both communities. For the first objective, I will analyze if higher social capital in one community that entails higher cooperation would lead to less or more cooperation in the other. For the second objective, I will analyze if the presence of social interactions between communities, even if random, leads to less or more cooperation in both communities and how it affects the welfare of both communities, as compared to when the social interaction between communities are absent.

This work has three major contributions to the literature. Firstly, the model contributes to the literature on cooperation in social dilemma situations by considering a public good game with thresholds, where agents can have a continuous strategy space of decision, instead of a binary one. To date, most theoretical and experimental work address the problem of linear public good games in which agents have a binary decision of contributing all or nothing. Second, I consider the case of multi-level cooperation, where cooperation may take place within and between communities. In particular, I consider asymmetric incentives arising from the fact that the upstream community has a first-mover advantage over the downstream, by having the first claim of resource. Thirdly, the model features spatial effects in two interdependent networks, each of which representing a community interacting with one another through a collective effort for the production of a CPR. Instead of having an unstructured population where random pairs of agents interact, I consider a structured population where the interactions of agents depend on their position in the own network as well as in relation to the other interdependent network. Thus, agent's decisions on extraction and investment efforts are influenced by how they view the behavior of members in their own networks as well as the behavior of members in the different network. 


\subsection{The model}

\subsubsection{Conceptual model}

Suppose that $N$ farmers are spread across two communities and share water infrastructure from which water is withdrawn for irrigation. Community 1 is located upstream and has $N_{1}$ farmers, whereas community 2 is located downstream and has $N_{2}$ farmers such that $N_{1}+N_{2}=N$. The upstream farmers have the first claim of water, and the downstream farmers get what remains. The infrastructure needs to be jointly maintained on a regular basis so that it can retain enough water for both communities. The upstream farmers, thus, have to depend on the downstream farmers to help maintain the shared infrastructure. Upstream and downstream farmers thus compete for water, but have a collective interest in maintaining the shared infrastructure. The stylized system under study is shown in Fig. 1.

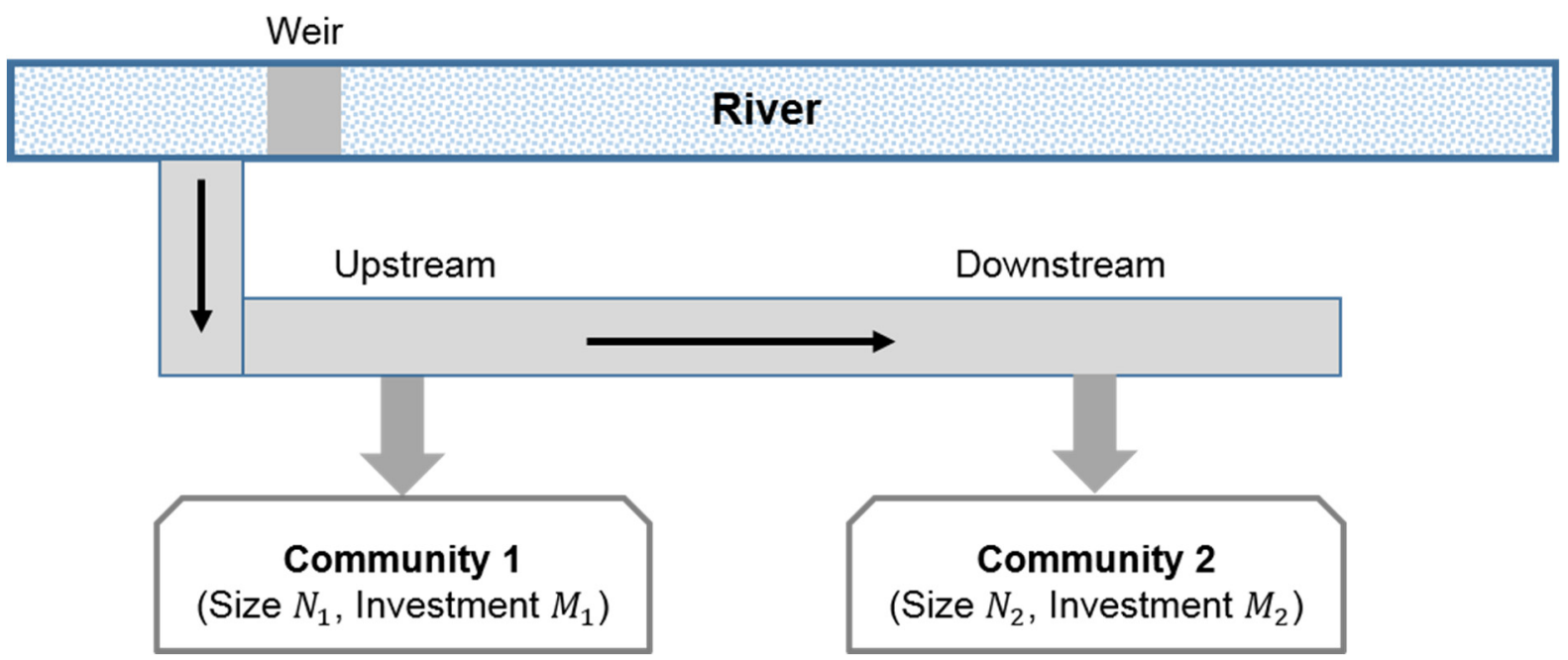

Fig. 1. Stylized system representation depicting the interactions between two communities who jointly harvest water as a common-pool resource and invest to the shared infrastructure as a public good. Community 1 gets the first claim of water, but depends on community 2 for a joint effort to maintain the infrastructure.

\subsubsection{A combined common-pool resource and public goods model}

The model features two types of decisions to be made by each farmer agent simultaneously, namely the water extraction and investment decisions. The investment decision is related to the shared water infrastructure. The infrastructure is a public good because the benefit of using it is non-excludable and using the infrastructure does not limit availability of usage by others. Investing to the infrastructure maintenance is costly for individuals, but the benefit of a well- 
maintained infrastructure can be enjoyed by everyone even if one does not contribute anything, giving rise to the so-called "public goods dilemma".

In real-world situations, agents can invest to the infrastructure maintenance by means of cash or labour. Denote $m_{i}$ as the individual investment effort with a fixed $\operatorname{cost}$ of $c_{3}$, the collective investment made by all agents is thus given by $M=\sum_{i=1}^{N} m_{i}$. The individual investment effort ranges from 0 to 1 , where 1 is full effort. The outcome of the investment is a collective good, i.e. water which is shared by all farmers in both communities. The relationship between collective investment contributed by all agents in both communities and the water availability $(S)$ is given by $S=\varepsilon(M) Q$, where $Q$ denotes water inflow into the system and $\varepsilon(M)$ is the infrastructure productivity which is a function of the collective investment $M$. I define $Q$ as a random variable with expected value $\mu_{Q}$ and standard deviation $\sigma_{Q}$, i.e. $Q \sim N\left(\mu_{Q}, \sigma_{Q}^{2}\right)$. The system productivity $\varepsilon(M)$ embodies the incentive structure of the public good model with thresholds $\mu_{1}$ and $\mu_{2}$. The minimum investment threshold $\left(\mu_{1}\right)$ depicts that a certain proportion of collective investment is needed for the maintenance of the infrastructure for it to produce the water, which is very common in the context of a small-scale irrigation system (Yu et al. 2015). Also, the maximum investment threshold $\left(\mu_{2}\right)$ entails that over-investing is inefficient and thus unnecessary. I have parameterised the model in such way that full contribution by one community is not enough to produce the maximum yield, and thus requires a joint effort from another community. The system productivity, as depicted in Fig. 2, can be expressed as a function of the collective investment $M(t)$ as

$$
\begin{cases}0 & \text { if } 0<M(t) \leq \mu_{1} \\ \left(M(t)-\mu_{1}\right) /\left(\mu_{2}-\mu_{1}\right) & \text { if } \mu_{1}<M(t) \leq \mu_{2} \\ 1 & \text { if } M(t)>\mu_{2}\end{cases}
$$

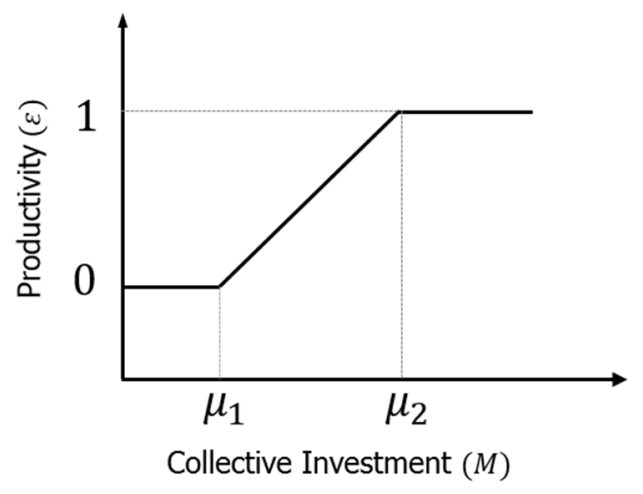

Fig. 2. Relationship between system productivity and collective investment. 
The second decision involves water harvesting. Water is a collective good and is referred to as a common-pool resource because the use of water by an agent reduces availability to others and it is costly, if not impossible, to exclude others from using it. I assume that farmers use only two inputs, land $l_{i}$ and water $w_{i}$ for the production of a final good $f\left(l_{i}, w_{i}\right)$. The land is a privately owned input and is assumed to have no market, but using land has a cost $c_{1}$. In the model, I consider the case of homogeneous land endowment, in which each agent has a land size of 1 unit. Water is a shared input and can be taken for free; however, the extraction effort for taking water from the common pool is associated with a cost (e.g. pumping cost or labor cost). Assume that each agent extracts water by exerting individual extraction effort $e_{i}$, with a fixed cost of $c_{2}$. The amount of water extracted by an agent in a particular community is proportional to the individual extraction effort, and is a fraction of the total water availability in that particular community. The individual extraction effort ranges from 0 to 1 , where 1 is full extraction effort. Due to the location advantage, agents in the upstream community get the first claim of water. The individual water extraction for the upstream community is thus given by $w_{i}^{u p}=\left(e_{i}^{u p} / E\right) S$, where $E$ is a parameter scaling the maximum capacity of all agents to extract all water, such that $E^{u p}+E^{\text {down }} \leq E$. This setting means that if the upstream community exerts the total extraction effort up to the maximum capacity, then all water available is used up by them, and nothing is left for the downstream. The value of $E$ is set at 400, implying that the if the upstream community make a full extraction effort $\left(E^{u p}=400\right)$, then all water is used up and nothing is left for the downstream. However, if the each agent in the upstream community makes a moderate extraction effort, e.g. $e_{i}^{u p}=0.5$, then the aggregate extraction effort in the upstream is 200 , meaning that collectively the upstream community takes only haft of the amount of water availability, leaving another half for the downstream. Obviously, if more water is left available for the downstream community, they are better off and also more capable to invest in water infrastructure. This setting thus gives rise to coupled social dilemmas between the two communities in terms of water extraction as well as investment to the infrastructure.

For the downstream community, the individual water extraction is given by $w_{i}^{\text {down }}=$ $\left[e_{i}^{\text {down }} /\left(E-E^{u p}\right)\right]\left[\left(S-W^{u p}\right]\right.$, where $W^{u p}$ is the aggregate water extraction in upstream community, and $E-E^{u p} \geq E^{\text {down }}$. The term $S-W^{\text {up }}$ stands for the total amount of water left after extraction by the upstream community, while $E-E^{u p}$ is the aggregate effort of the downstream - relative to the maximum capacity of all agents - that leads to water left for the downstream to be all extracted. If the upstream community collectively takes all water such that 
$E=E^{u p}$ or $S=W^{u p}$, then individual water extraction in the downstream $\left(w_{i}^{\text {down }}\right)$ becomes zero. Further, if the upstream community takes too much water such that the extractive capacity of the downstream $\left(E^{\text {down }}\right)$ is greater than the aggregate effort that lead to the remained water to be all extracted $\left(E-E^{u p}\right)$, the individual extraction effort of the downstream agent is scaled down proportionally with a factor $\left(E-E^{u p}\right) / E^{\text {down }}$, such that the downstream agent who exerts high extraction effort gets high amount of water. Likewise, if the total amount of water left in the downstream is low, individually and collectively the downstream agents exert low extraction effort proportional to their extractive capacity. For a sales price $p$ of the final good, the profit of agent $i$ at time $t$ is given by

$$
\pi_{i}(t)=p f\left(l_{i}, w_{i}(t)\right)-c_{1} l_{i}-c_{2} e_{i}(t)-c_{3} m_{i}(t)
$$

Assume that the production of the final good is represented by a Constant Elasticity of Substitution (CES) function, i.e. $f=\rho\left(w_{i}^{\alpha}+l_{i}^{\alpha}\right)^{1 / \alpha}$, where $\rho$ is skill or productive factor and $\alpha<1$ measures the degree of complementarity between land and water inputs. This function covers the whole continuum of alternative combinations of inputs from perfect complementarity to perfect substitution. For example, when $\alpha \rightarrow 1$, the function exhibits perfect substitution, meaning it is possible to use either input alone to achieve the same level of output. When $\alpha \rightarrow 0$, the function exhibits some degree of substitution between inputs, i.e. it is possible to use more of one input and less of the other to obtain the same level of output. When $\alpha \rightarrow$ $-\infty$, the function exhibits perfect complementarity between inputs, i.e. the production is possible only if all inputs are used. For the model, I assume that agents have a fixed land endowment which is exogenously given. I also assume that there is some degree of complementarity between land and water inputs, i.e. $0<\alpha<1$. Finally, the profit of agent $i$ at time $t$ can be expressed as

$\pi_{i}(t)=p \rho\left(w_{i}^{\alpha}+l_{i}^{\alpha}\right)^{1 / \alpha}-c_{1} l_{i}-c_{2} e_{i}(t)-c_{3} m_{i}(t)$

\subsubsection{Social dynamics}

\section{Reputation dynamics}

Reputation is a key mechanism for cooperation among unrelated individuals, though it involves some uncertainties, e.g. regarding accessing to information about reputation of other agents (Nowak and Sigmund 1998). In some cases, agents are assumed to have full information about reputation of all other agents. In other cases, agents have only partial information about reputation, e.g. the reputation of immediate neighbours. 
In the model, self-image or reputation is associated with cooperative acts. Cooperating increases one's self-image, while defecting decreases it. Because cooperative acts are determined by both investment and extraction levels, the self-image is thus a function of investment and extraction levels. This conceptualization thus resembles the notion of conditional cooperation, as agents are more inclined to cooperate if others do so as well. Using more water generates higher income, but taking too much water compared to the social norm is morally bad and can result in losing reputation. Under-harvesting of water signals good behaviour and thus get rewarded if one does so. In a similar vein, investing to infrastructure maintenance is costly, but investing too little or nothing compared to the social norm is considered morally bad. Over-investing, however, is encouraged. If one does so, one will get rewarded. The self-image of agent $i$ at time $t$ in each community can be expressed as follows.

$g_{i}(t)=\omega_{1}\left(\bar{e}-e_{i}(t)\right)\left|\bar{e}-e_{i}(t)\right|+\omega_{2}\left(m_{i}(t)-\bar{m}\right)\left|m_{i}(t)-\bar{m}\right|$

where $\bar{e}=\left(1 / N_{1}\right) \sum_{i=1}^{N_{1}} e_{i}$ and $\bar{m}=\left(1 / N_{1}\right) \sum_{i=1}^{N_{1}} m_{i}$ represents the average water extraction and investment efforts respectively in community 1 , which is considered the social norm in the that community. $N_{1}$ is the total number of agents in that particular community. The parameters $\omega_{1}$ and $\omega_{2}$ can be considered as social cohesion coefficients in relation to extraction and investment respectively; the higher they are, the greater utility loss inflicted for taking too much water or investing too little to infrastructure maintenance, as compared to the social norm. For simplicity, I assume that the social cohesion in relation to extraction is the same as the social cohesion in relation to investment. For some simulations which aim to study different social structures in the two communities, I allow these coefficients to be different between the upstream and downstream communities.

In this formulation, over-extracting or under-investing relative to the social norm results in reputation loss. Also, under-extracting or over-investing results in gains of reputation. Overall, this function depicts that cooperation increases self-image, while defection decreases it. In addition, aligning one's behaviour with the social norm has no effects on utility. The self-image score ranges from -1 to 1 , where -1 is realized when the agent's behavior is in the extreme negative side relative to the social norm, and 1 is when the agent behavior in the extreme positive side relative to the social norm. The image score is equal to zero when the agent fully aligns with the social norm. 
The performance of agent $i$ is represented by his utility $u_{i}(t)$, which reflects how well agent $i$ is doing economically and how good agent $i$ is doing morally. Suppose that $\bar{\pi}$ and $\bar{g}$ are exogenous profit and reputation respectively. I assume that the utility of agent $i$ exhibits Cobb Douglas preferences, which can be written as

$u_{i}(t)=\left(\bar{\pi}+\pi_{i}(t)\right)^{\beta}\left(\bar{g}+g_{i}(t)\right)^{1-\beta}$

where $\beta$ represents the preference for profits.

\section{Strategy updating and social learning}

Social learning is a factor that facilitates cooperation to spread within communities through a reputation mechanism. Here, I assume that the social interactions within the community enable an agent to learn about water extraction levels of his neighbours taking place in two separated $d \times d$ square lattices where $d$ is the lattice dimension. Each lattice represents social interactions in the upstream and the downstream community. Suppose that each agent occupies a cell of the square lattice, so that two fully-occupied lattices contain $2 d^{2}$ agents. Assume that agent $i$ does not have perfect information about his decision made and considers changing his strategy regarding water extraction and investment to maintenance at time $t+1$ by learning from what his immediate neighbours do at time $t$. To do so, agent $i$ compares his utility with the utility of his four immediate neighbours, without considering the behavior of members in the other community-implying that no interactions between communities take place. In the context of resource management, social interactions among agents take place inside and outside own communities.

In some simulations, I also consider the case where a focal agent interacts with his four immediate neighbours as well as with one random partner from another community. I assume that the interactions among agents from the same community occur more frequently than those with members from the different community. Agent $i$ copies the strategy that yields the highest utility, say from agent $j$ with a probability $P$. The probability $P$ is an increasing function of the utility difference $u_{j}-u_{i}$. I use a logistic function to model the adaptation probability as follows

$$
P\left\{\left(e_{i} \rightarrow e_{j}\right) \&\left(m_{i} \rightarrow m_{j}\right)\right\}=\frac{1}{1+\exp \left[-\lambda\left(u_{j}-u_{i}\right)\right]},
$$

where $\lambda \geq 0$ can be considered as imitation strength which measures how strongly the utility difference influences the decision of an agent to switch to a better-off strategy. $\lambda \rightarrow 0$ or $u_{j}=$ $u_{i}$ means agent $i$ tosses the coin to decide if he imitates the strategy of agent $j . \lambda \rightarrow+\infty$ 
corresponds to high imitation strength, meaning that a successful strategy is always imitated. Small $\lambda$ depicts low imitation strength, meaning that a successful strategy is imitated with a low probability. For the simulations that consider social interactions between communities, I assume that the imitation strength within a community $\left(\lambda_{1}=10\right)$ - the likelihood of an agent updating the strategies following his best-performing immediate neighbour - is higher than the imitation strength between communities $\left(\lambda_{2}=5\right)$ - the likelihood of an agent updating the strategies following his random partner of a different community.

\section{Model variables and parameter values and definition}

Table 1: Model variables and parameter at default values

\begin{tabular}{|c|c|c|}
\hline Parameters & Definition & "Value \\
\hline $\bar{N}$ & Total number of households & 800 \\
\hline$N_{1}$ & Total number of households in upstream commune & 400 \\
\hline$N_{2}$ & Total number of households in downstream village & 400 \\
\hline$T$ & Simulation length & 100 \\
\hline $\bar{Q}$ & Mean inflow & 200 \\
\hline$\sigma_{Q}$ & Standard deviation of inflow & 20 \\
\hline$e_{i}(0)$ & Individual water extraction effort at $t=0$ & $\mathcal{N}\left(0.5,0.1^{2}\right)$ \\
\hline$m_{i}(0)$ & Individual investment effort at $\mathrm{t}=0$ & $\mathcal{N}\left(0.5,0.1^{2}\right)$ \\
\hline E & Maximum capacity of all agents to extract all water & 400 \\
\hline$l$ & Individual land endowment & 1 \\
\hline$\mu_{1}$ & Minimum investment threshold & 300 \\
\hline$\mu_{2}$ & Maximum investment threshold & 600 \\
\hline$p$ & Sales price & 1 \\
\hline$c_{1}$ & Unit cost of farming per unit land & 0.5 \\
\hline$c_{2}$ & Unit cost of investment effort & 0.5 \\
\hline$c_{3}$ & Unit cost of water extraction effort & 0.5 \\
\hline$\omega$ & Social cohesion coefficient in relation extraction & 0.5 \\
\hline$\omega_{2}$ & Social cohesion coefficient in relation investment & 0.5 \\
\hline$\lambda_{1}$ & Imitation strength within community & 10 \\
\hline
\end{tabular}




$\begin{array}{lll}\lambda_{2} & \text { Imitation strength between community } & 5 \\ \alpha & \text { Degree of complementarity between land and water } & 0.6 \\ \bar{\pi} & \text { Exogenous profit } & 0.1 \\ \bar{g} & \text { Exogenous reputation } & 0.1 \\ \beta & \text { Profit preference } & 0.7\end{array}$

\subsection{Results}

\subsubsection{Evolution of cooperation without encounters between communities}

Fig. 3 shows the evolution of cooperation in which social interactions between communities are absent. Agents are thus learning and updating strategies following the best performing neighbours in their own community. The model is run 50 times repeatedly to account for stochasticity, starting from a random configuration of extraction and investment efforts, normally distributed with means of 0.5 and standard deviations of 0.1 , and with default parameter values. Overall, the model reaches only one equilibrium for investment, i.e. low investment efforts in both communities (fig. $3 b \& 3 d$ ). While the extraction effort is high (between 0.6 and 0.7 ) in the upstream community (Fig. 3a), the extraction effort is low (between 0.3 and 0.4 ) in the downstream (Fig. 3c). This reflects the upstream community having the first claim of water and the downstream getting what remains. Despite significantly higher extraction effort (Fig. 3e) and slightly higher investment effort (Fig. 3f) in the upstream community, this does not translate directly into relatively higher aggregate profit, as compared to that of the downstream (Fig. $3 \mathrm{i} \& 3 \mathrm{j}$ ). This is because not enough aggregated investment is collected from both communities, resulting in low water availability, making resource extraction more costly and thus less profitable for agents who exert high extraction effort. This result highlights a social dilemma situation between upstream and downstream communities, in which individual self-interested behavior of the upstream taking more resource is economically better off initially, but over time leads to an undesirable state for all. 
(a)

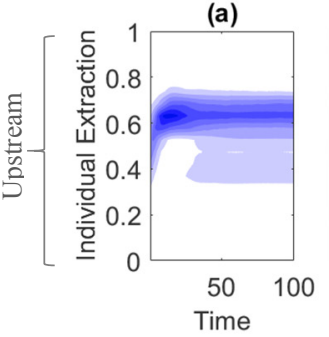

(c)

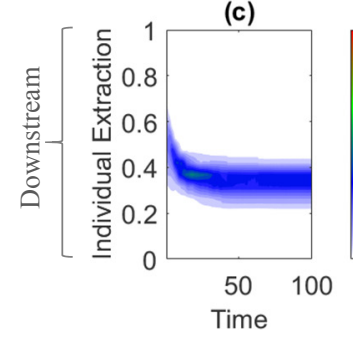

(b)

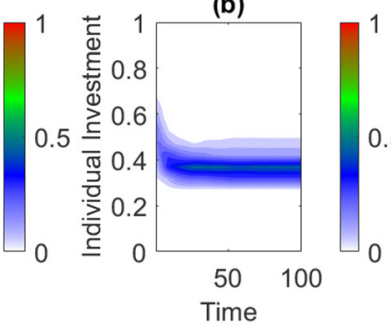

(d)

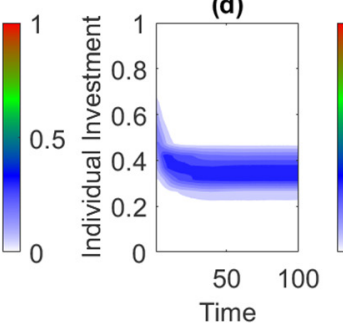

(e)

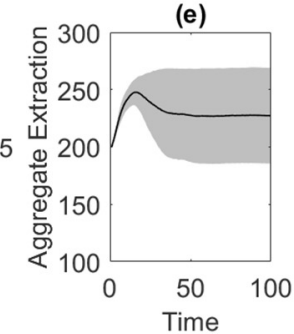

(g)

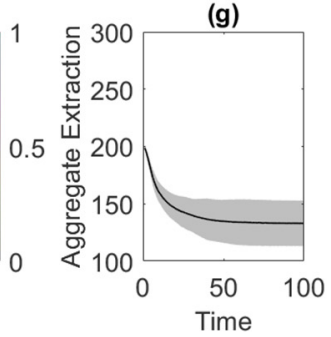

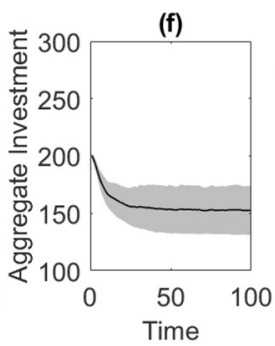

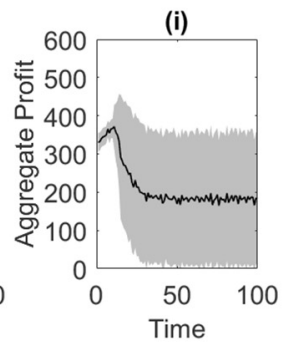

(h)
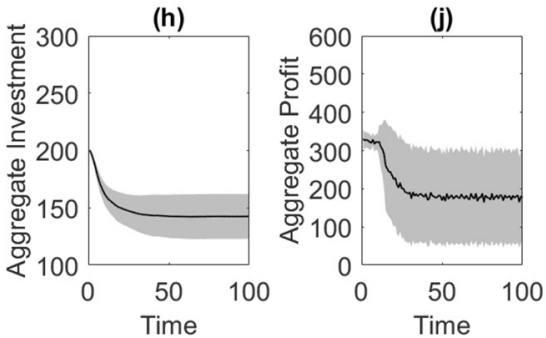

Fig. 3. Temporal evolution of extraction and investment effort at default parameter values and under the condition where social interactions between communities are absent. The top panel represents upstream effort, while the bottom panel represents the downstream effort. X-axis stands for simulation time. Yaxis represents frequency distribution of extraction and investment efforts among agents at each time step over 50 repeated runs (a-d), and aggregate values averaged over 50 repeated runs (e-j). Colorbar represents the proportion. The simulation started with random extraction and investment effort between 0 and 1 . Agents copy the extraction and investment effort of the best performing neighbour in their own community.

\subsubsection{Social interactions between communities promote cooperation}

In the context of resource management, social interactions among agents take place inside and outside of own communities. Here, I assume that a focal agent interacts with his four immediate neighbours as well as with one random partner from another community. Fig. 4 depicts the evolution of cooperation in which social interactions between communities are considered. Overall, the interactions of agents between communities promote cooperation in both communities, mainly through lowering the extraction effort in the two communities. While the aggregate investment effort in both communities (Fig. 4b \& 4d) remains similar as compared to the case in which the interactions between communities are absent (Fig. 3), the extraction effort is much lower. In the upstream community, the population is almost equally spread into two groups: one with high extraction effort and another with very low extraction effort (Fig. 4a). In the downstream, the low extracting population dominates (Fig 4c). Lower aggregate extraction effort in both communities (Fig. 4e \& 4g) results in higher aggregate profits (Fig. 4i $\& 4 \mathrm{j})$ since it is collectively more profitable to extract less if the amount of water availability is low due to low aggregate investment effort (Fig. $4 \mathrm{f} \& 4 \mathrm{~h}$ ). This result highlights the effects of 
social learning in promoting overall welfare through interactions between communities, even if the interactions are less frequent and random.

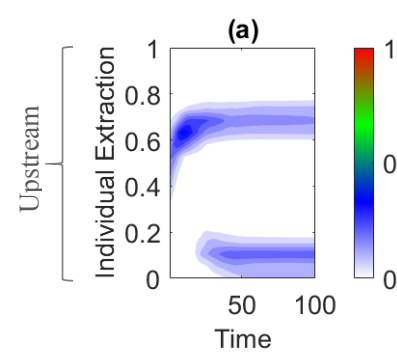

(c)

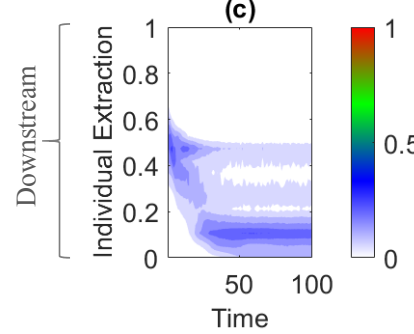

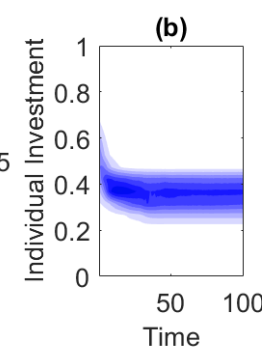

(d)

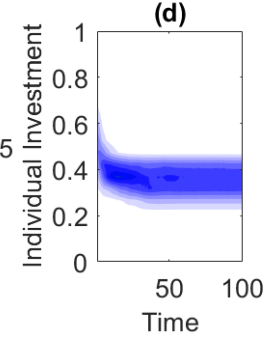

(e)

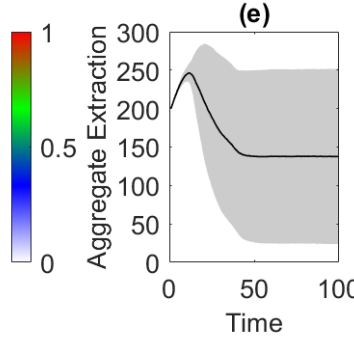

(g)
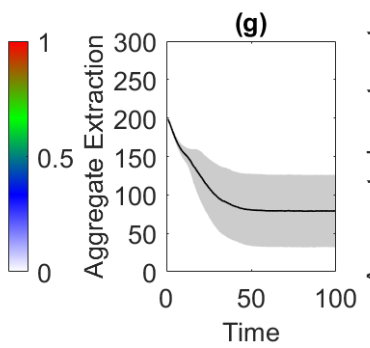
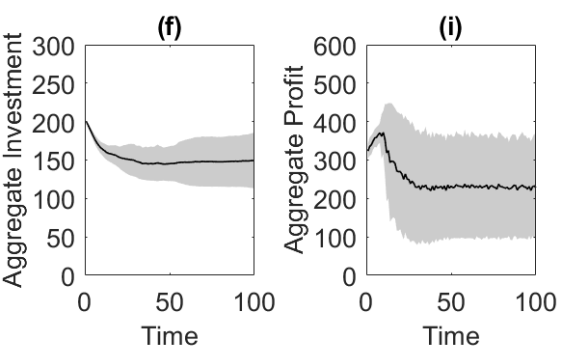

(h)
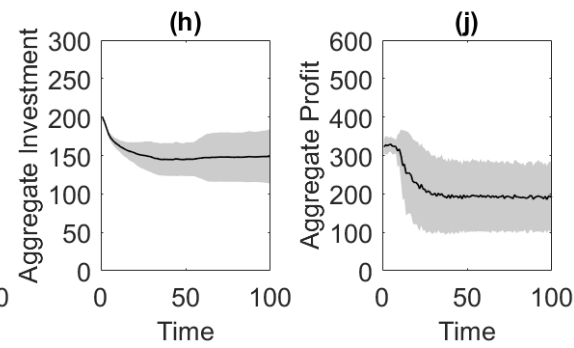

Fig. 4.Temporal evolution of extraction and investment effort at default parameter values and under the condition where random social interactions between communities take place. The top panel represents upstream effort, while the bottom represents the downstream effort. X-axis stands for simulation time. $\mathrm{Y}$-axis represents frequency distribution of extraction and investment efforts among agents at each time step over 50 repeated runs (a-d), and aggregate values averaged over 50 repeated runs (e-j). Colorbar represents the proportion. The simulation started with random extraction and investment effort between 0 and 1 . The focal agent compares his performance with that of his immediate neighbours and with a random partner of the other community, and copies the extraction and investment efforts of the best performing partner.

Fig. 5 gives details of the model behavior at equilibrium between the case in which social interactions between the two communities are absent (grey) and the case in which random interactions between the communities are considered (black). Obviously, I observe a much improved cooperation in both communities, particularly through much lower extraction effort, when the social interactions between communities are present (Fig. 5a \& 5b). The overall enhancement of cooperation in both communities due to the presence of inter-community interactions results in higher aggregate payoff (Fig. 5c \& 5d) and utility (Fig. 5e \& 5f) in both communities, as compared to the case where the interactions are not considered. 
(a)

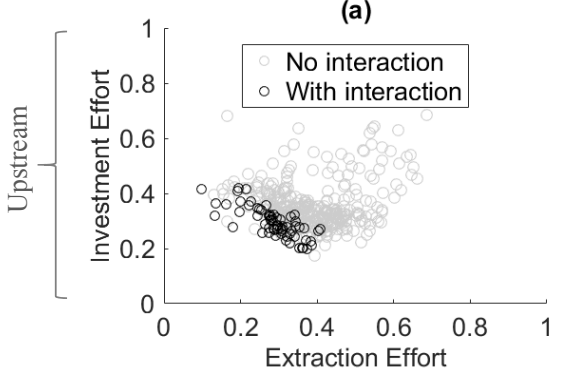

(b)

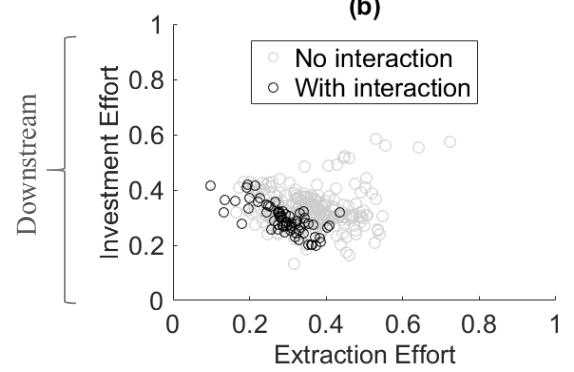

(c)

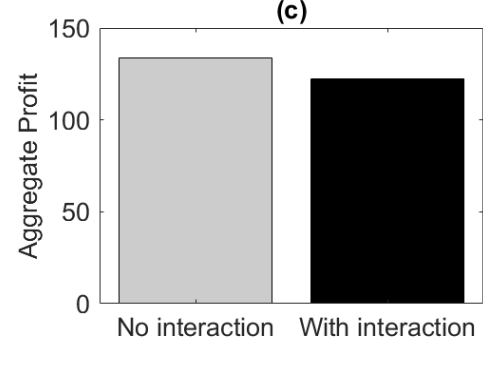

(d)

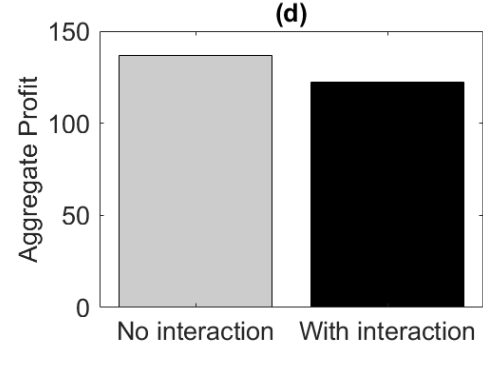

(e)

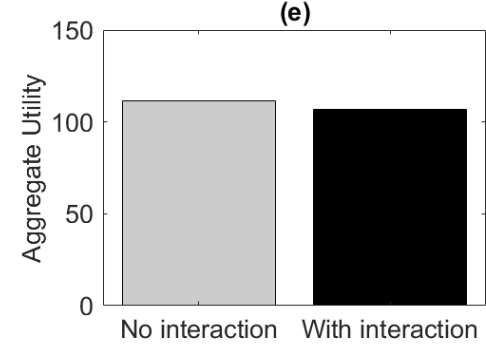

(f)

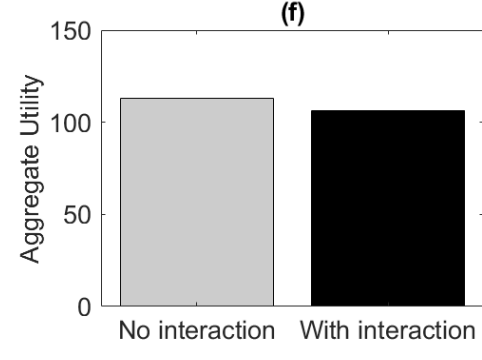

Fig. 5. Agents' profile characterised by extraction and investment efforts at equilibrium state (a-b), and associated aggregate payoff (c-d) as well as aggregate utility for the cases in which (i) there is no social interaction between communities (grey), and (ii) a focal agent has interaction with one random partner from a different community (black). The top panel represents upstream effort, while the bottom represents the downstream effort. The model is run until reaching the equilibrium state for 50 repeated runs to account for stochasticity.

\subsubsection{High social cohesion upstream leads to more cooperation downstream}

Social cohesion binds the community members together and allows them to act cohesively towards a joint purpose. High social cohesion means the connectedness or relationship among members is strong, which implies that the behavior of community members are more easily observable. In the context of this model, this implies that deviating from the social norms in a highly cohesive community will lead to greater loss of reputation. Practically, I set the values of the model parameters for social cohesion to 2 , instead of 0.5 as in the default condition.

Fig. 6 shows that higher social cohesion in the upstream community leads to much improved cooperation in both communities. In particular, higher social cohesion in the upstream induces higher aggregate investment in both communities, resulting in more water availability. Agents in both communities thus exert higher extraction efforts, and generate more profit for extra efforts. It's worth to note that under this condition the downstream community is generally doing economically better. While the aggregate extraction efforts in both communities are similar, the investment effort is higher in the upstream, making the upstream less profitable for their higher investment. 
While high social cohesion in the upstream leads to much improved cooperation overall, this is not the case when the social cohesion is high in the downstream (Fig. 7). Higher social cohesion in the downstream only slightly increases investment efforts in both the downstream and upstream communities, which is not enough to produce significantly more water for agents in both communities to extract. The marginal increases in investment as well as water availability lead to slightly higher aggregate profits in both communities.
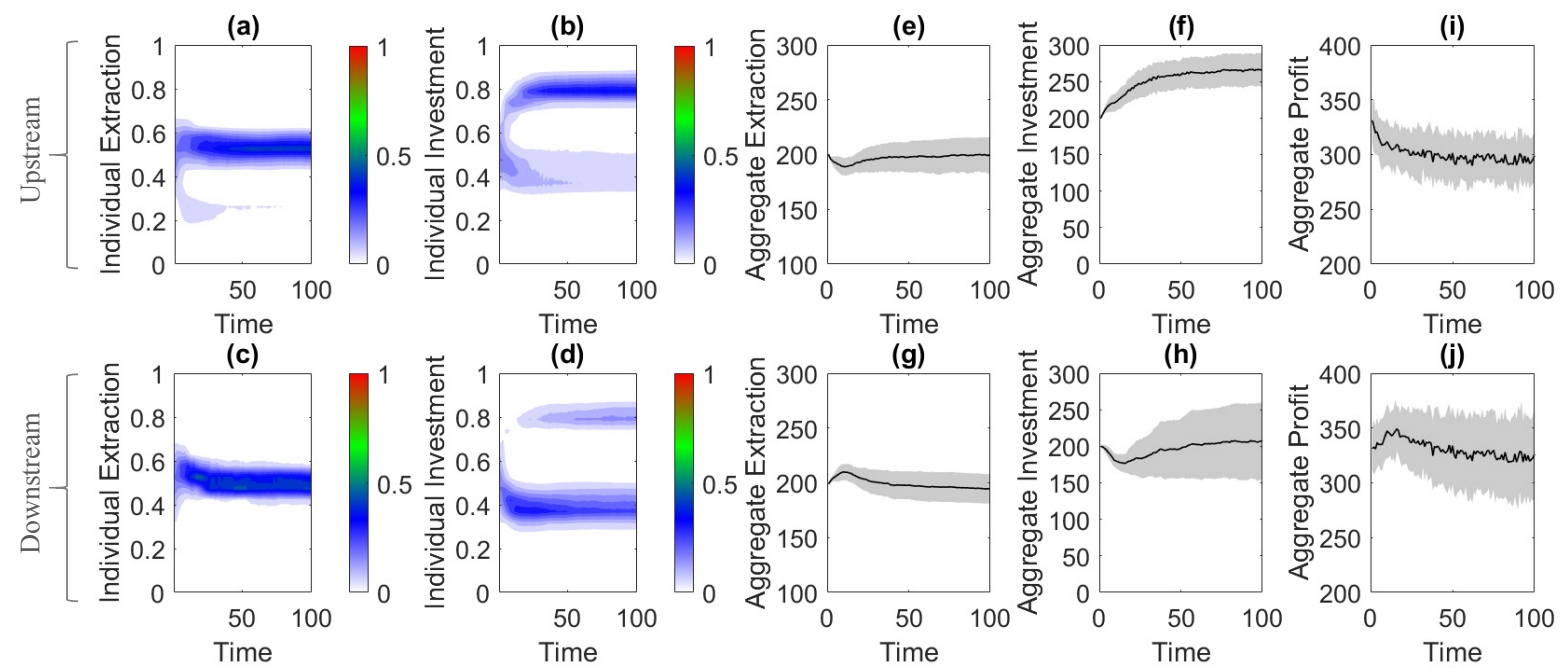

Fig. 6. Effects of social cohesion upstream on investment and extraction efforts. The social cohesion coefficient in the upstream is set higher $\left(\omega_{1}^{u p}=2 ; \omega_{2}^{u p}=2\right)$, while other variables are kept at default values. The top panel represents upstream effort, while the bottom represents the downstream effort. Xaxis stands for simulation time. $\mathrm{Y}$-axis represents frequency distribution of extraction and investment efforts among agents at each time step over 50 repeated runs (a-d), and aggregate values averaged over 50 repeated runs (e-j). Colorbar represents the proportion of agents relative to the size of the community. 

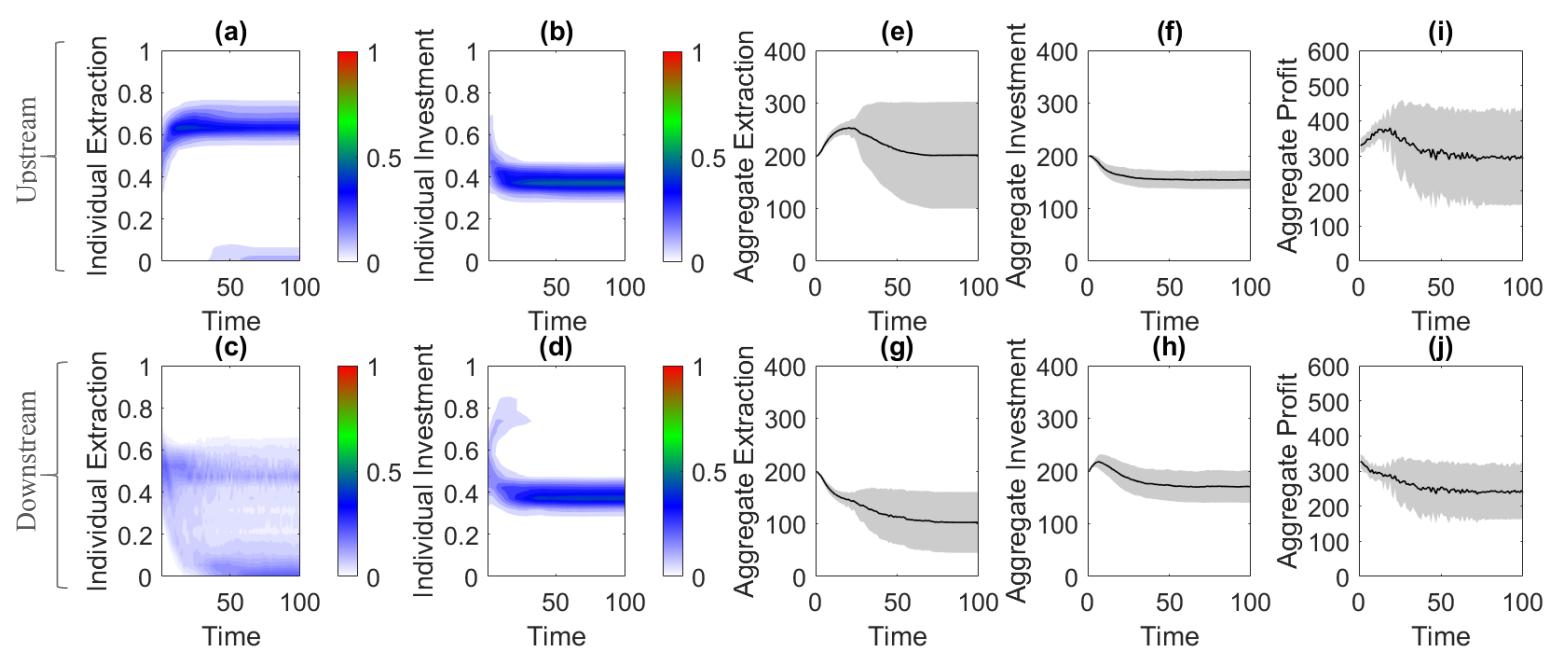

Fig. 7. Effects of social cohesion downstream on investment and extraction efforts. The social cohesion coefficient in the downstream is set higher $\left(\left(\omega_{1}^{\text {down }}=2 ; \omega_{2}^{\text {down }}=2\right)\right)$, while other variables are kept at default values. The top panel represents upstream effort, while the bottom represents the downstream effort. X-axis stands for simulation time. Y-axis represents frequency distribution of extraction and investment efforts among agents at each time step over 50 repeated runs (a-d), and aggregate values averaged over 50 repeated runs (e-j). Colorbar represents the proportion of agents relative to the size of the community.

\subsubsection{Effects of key parameters on extraction and investment efforts}

I run a series of simulations to study the effects of key parameters of the model on extraction and investment efforts of agents (Fig.8). First, higher social cohesion in relation to extraction upstream-meaning that it is socially costly to deviate one's extraction effort from the social norm - slightly lowers extraction effort in the upstream and more investments in both communities (Fig. 8a-d). However, when this coefficient is higher in downstream, it does not impact extraction and investments effort in the upstream (Fig. 8i-1). Notably, this only leads to slightly lower extraction effort and slightly higher investment effort in the downstream, suggesting that reputation dynamics in the downstream does not induce cooperation in the upstream. On the other hand, varying degree of social cohesion in relation to investment seems to be much more responsive (Fig. 8e-h). When the social cohesion coefficient in relation to investment upstream is higher, the investment effort upstream is also higher, while the investment effort downstream remains the same. However, this is true only when the coefficient is not too high. At the same time, extraction efforts in both communities are higher since there is more water availability due to higher investment. Similarly, higher social cohesion in relation to investment downstream leads to more investment downstream, but there is no change in investment effort in the upstream (Fig. 8m-p). However, the increased investment in the 
downstream is associated with higher extraction effort in both communities, since more water is available for extraction. Overall, the results suggest that the reputation dynamics in the upstream could have more impact on cooperation than that in the downstream could have, especially when the social cohesion is not too high.
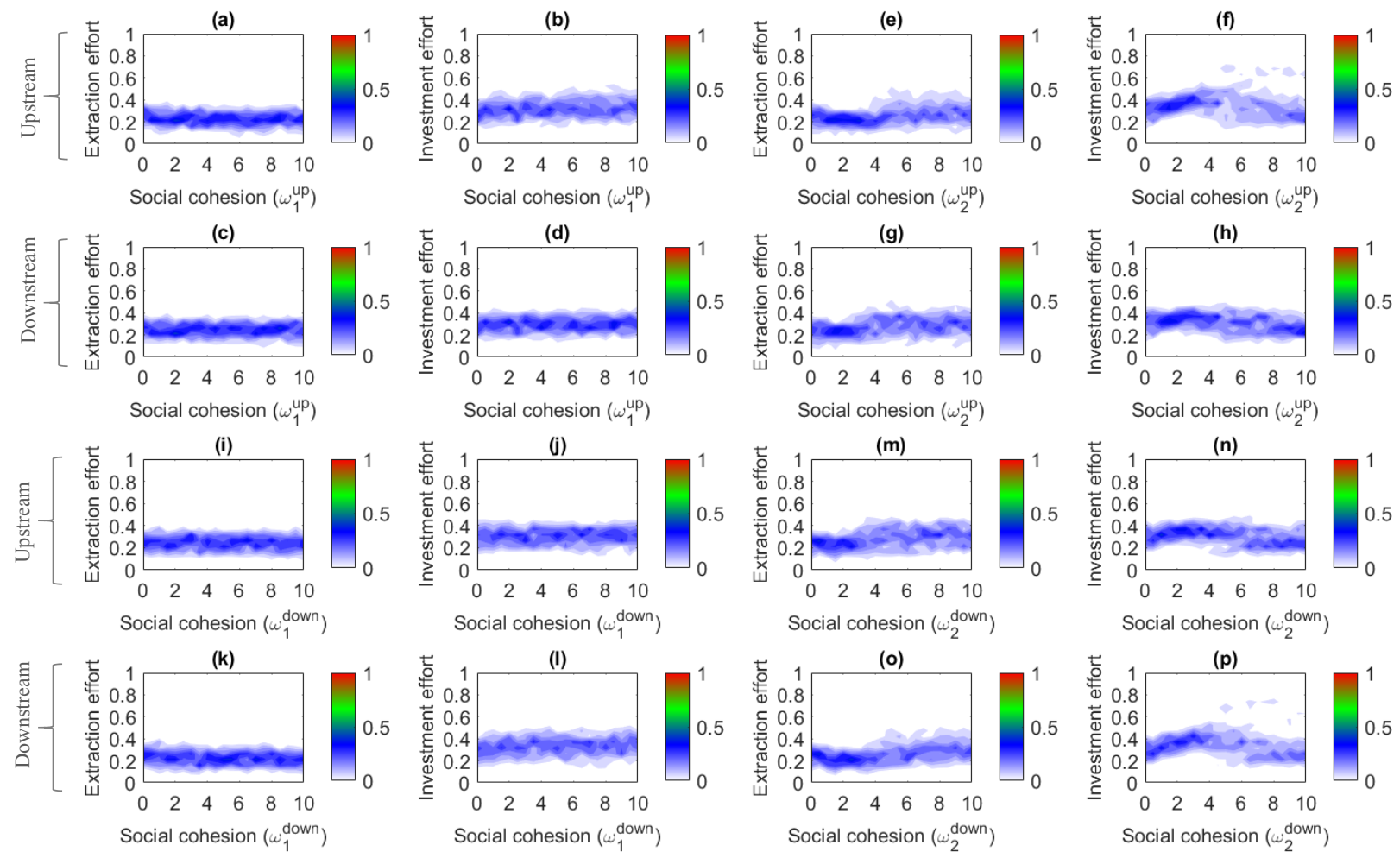

Fig. 8.One-at-a-time sensitivity plot of key model parameters against extraction and investment efforts at equilibrium. To account for stochasticity, I run the model 20 times repeatedly for each single value of the parameter. $\mathrm{X}$-axis represent parameter space. $\mathrm{Y}$-axis represents frequency distribution of extraction and investment efforts at equilibrium state.

\subsection{Discussions and conclusion}

I have developed an evolutionary agent-based model to analyse under what conditions cooperation emerges and spreads across two communities who share water as a common-pool resource and infrastructure as a public good. Agents make decisions on levels of extraction and investment efforts over time, learning from their immediate neighbours as well as from one random partner from a different community when the social interactions between communities are considered. The simulations start from random configuration of investment and extraction efforts whose values are heterogeneous. While taking more resource or investing less to its production as compared to the social norm makes an agent economically better off in the short- 
term, doing so erodes his self-image which can potentially inflict loss on his economic performance in the long-term.

The model results show that increased cooperation in the upstream community leads to more cooperation in the downstream community, but the opposite is not true-increased cooperation in the downstream has minimal impact on cooperation in the upstream. This is not the case, however, if the social interactions between communities are not considered (See Fig. S2-3, supplementary material), and reputation mechanism is absent (Fig. S1, supplementary material). This result is mainly driven by the asymmetric incentive between the two communities, in which the level of cooperation in the downstream as well as the overall cooperation are conditional upon behavior as well as the social cohesion of the upstream. The model results also show that social capital, in the form of social interactions between the two communities even if it occurs randomly, can moderate the effect of the asymmetric incentive that allows the upstream to dictate the behavior as well as the performance of the system. This is especially the case when the social capital, in the form of social cohesion, in the upstream community is strong enough. Finally, the model suggests that fostering social capital between communities could be key to overcoming coupled social dilemmas among two communities jointly harvesting the common-pool resources and at the same time contributing towards the production of the resource. While the model is theoretically based, which naturally limits potential for predictions about real-world cases, its structure and setting captures very well a typical case of self-governance of the local water resource in two communities which share a small-scale irrigation system. 


\section{Acknowledgments}

This work benefits greatly from several rounds of comments and discussions with Andries Richter and Matthias Wildemeersch. The earlier draft was mainly written, presented, and discussed with researchers at IIASA, especially in the Advanced Systems Analysis Department. I thank Nikita Strelkovsky and Elena Rovenskaya for providing encouragement and support, especially on model implementation, during my stay at IIASA. I am indebted to the Young Scientists Summer Program (YSSP) for providing resources and stimulating environment, as well as the funding from Netherlands Organization for Scientific Research (NWO). I also gratefully acknowledges funding from the European Commission through the Alfabet project under the EU Erasmus Mundus Program. 


\section{Reference}

Botelho, Anabela, Ariel Dinar, Lígia M.Costa Pinto, and Amnon Rapoport. 2015. "Promoting Cooperation in Resource Dilemmas: Theoretical Predictions and Experimental Evidence." Journal of Behavioral and Experimental Economics 54: 40-49. https://doi.org/10.1016/j.socec.2014.11.007.

Brekke, Kjell Arne, Snorre Kverndokk, and Karine Nyborg. 2003. “An Economic Model of Moral Motivation.” Journal of Public Economics 87 (9-10): 1967-83. https://doi.org/10.1016/S0047-2727(01)00222-5.

Dietz, Thomas, Elinor Ostrom, and Paul C. Stern. 2003. "The Struggle to Govern the Commons." Science 302 (5652): 1907-12. https://doi.org/10.1126/science.1091015.

Gardner, Roy, Elinor Ostrom, and James M. Walker. 1990. "The Nature of Common-Pool Resource Problems." Rationality and Society 2 (3): 335-58.

Nhim, Tum, and Andries Richter. 2021. "Path Dependencies and Institutional Traps in Water Governance- Evidence from Cambodia."

Nowak, Martin A. 2006. "Five Rules for the Evolution of Cooperation." Science 314 (5805): $1560-63$.

Nowak, Martin A., and Karl Sigmund. 1998. "Evolution of Indirect Reciprocity by Image Scoring." Nature 393 (6685): 573-77. https://doi.org/10.1038/31225.

Ostrom, Elinor. 2009. “A General Framework for Analyzing Sustainability of SocialEcological Systems.” Science 325 (5939): 419-22.

—. 2010. "Beyond Markets and States : Polycentric Governance of Complex Economic Systems." American Economic Review 100 (3): 641-72.

Pahl-Wostl, Claudia. 2009. "A Conceptual Framework for Analysing Adaptive Capacity and Multi-Level Learning Processes in Resource Governance Regimes.” Global Environmental Change 19 (3): 354-65. https://doi.org/10.1016/j.gloenvcha.2009.06.001. Perc, Matjaž, Jesús Gómez-Gardeñes, Attila Szolnoki, Luis M. Floría, and Yamir Moreno. 2013. "Evolutionary Dynamics of Group Interactions on Structured Populations: A Review." Journal of the Royal Society Interface 10 (80). https://doi.org/10.1098/rsif.2012.0997.

Robinson, Elva J.H., and Jessica L. Barker. 2017. "Inter-Group Cooperation in Humans and Other Animals.” Biology Letters 13 (3). https://doi.org/10.1098/rsbl.2016.0793.

Rockström, Johan, Will Steffen, Kevin Noone, Åsa Persson, F. Stuart Chapin, Eric Lambin, 
Timothy M. Lenton, et al. 2009. "Planetary Boundaries: Exploring the Safe Operating Space for Humanity." Nature 461 (7263): 472-75. https://doi.org/10.1038/461472a.

Sethi, Rajiv, and E Somanathan. 1996. "The Evolution of Social Norms in Common Property Resource Use." The American Economic Review 86 (4): 766-88.

Solstad, Jan Tore, and Kjell Arne Brekke. 2011. "Does the Existence of a Public Good Enhance Cooperation among Users of Common-Pool Resources?" Land Economics 87 (2): 335-45. https://doi.org/10.1353/lde.2011.0023.

Tavoni, Alessandro, Maja Schlüter, and Simon Levin. 2012. "The Survival of the Conformist: Social Pressure and Renewable Resource Management." Journal of Theoretical Biology 299: 152-61.

Veldkamp, T.I.E., Y. Wada, J.C.J.H. Aerts, P. Döll, S. N. Gosling, J. Liu, Y. Masaki, et al. 2017. "Water Scarcity Hotspots Travel Downstream Due to Human Interventions in the 20th and 21st Century." Nature Communications 8. https://doi.org/10.1038/ncomms15697.

Walker, Brian, Scott Barrett, Stephen Polasky, Victor Galaz, Cari Folke, Gustav Engström, Frank Ackerman, et al. 2009. "Looming Global-Scale Failures and Missing Institutions." Science 325 (5946): 1345-46. https://doi.org/10.1126/science.1175325.

Wang, Zhen, Attila Szolnoki, and Matjaž Perc. 2013. "Interdependent Network Reciprocity in Evolutionary Games.” Scientific Reports 3: 1-7. https://doi.org/10.1038/srep01183.

Yu, David J., Murad R. Qubbaj, Rachata Muneepeerakul, John M. Anderies, and Rimjhim M. Aggarwal. 2015. "Effect of Infrastructure Design on Commons Dilemmas in Social-ecological System Dynamics." Proceedings of the National Academy of Sciences 112 (43): 13207-12. https://doi.org/10.1073/pnas.1410688112. 


\section{Supplementary materials}

\section{Without reputation effects, cooperation in both communities collapses}

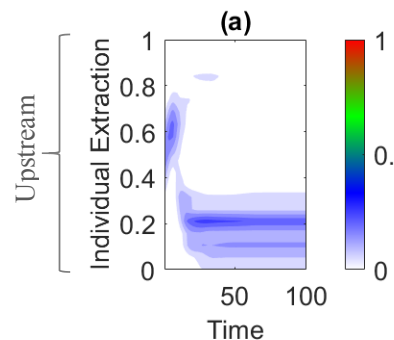

(c)

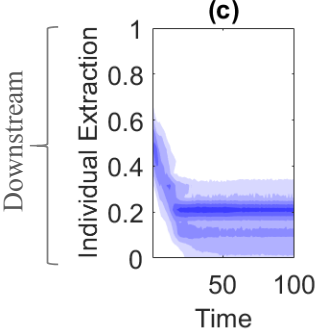

(b)

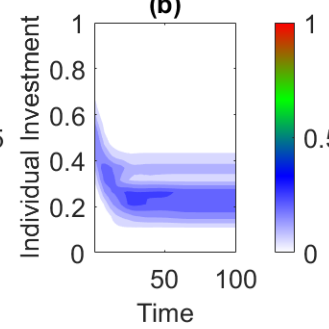

(d)

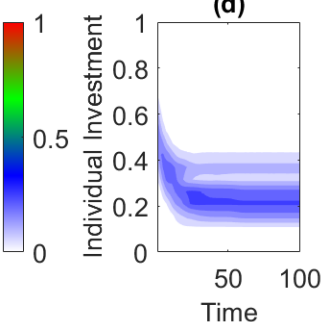

(e)

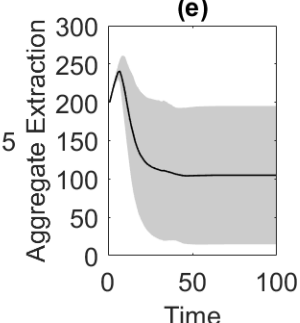

(g)
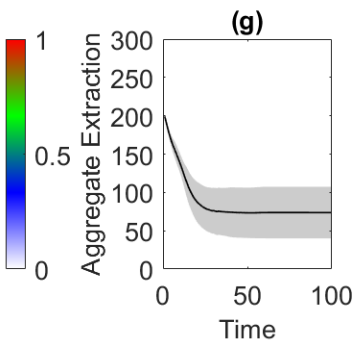

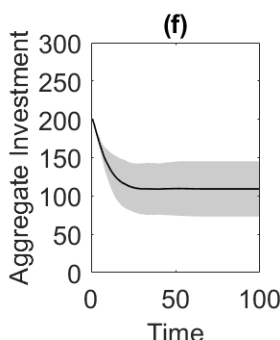

(h)

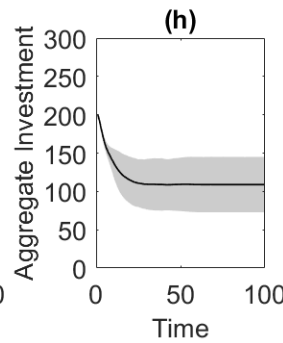

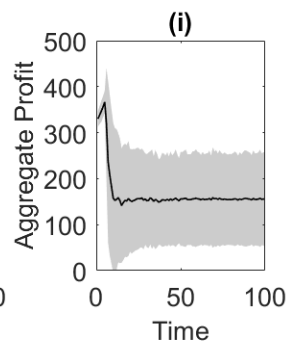

(j)

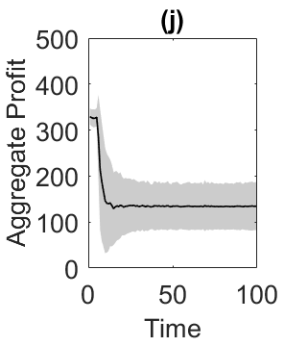

Fig.S1. Effects of reputation on investment and extraction efforts. The social cohesion coefficientshigher coefficients implying higher reputation effect—is set critically low $\left(\omega_{1}=\omega_{2}=0.01\right)$, while other variables are kept at default values. The top panel represents upstream effort, while the bottom represents the downstream effort. X-axis stands for simulation time. Y-axis represents frequency distribution of extraction and investment efforts among agents at each time step over 50 repeated runs (a-d), and aggregate values averaged over 50 repeated runs (e-j). Colorbar represents the proportion of agents relative to the size of the community.

\section{Without social interactions between communities, higher cooperation in the} upstream does not lead to more cooperation in the downstream, and vice versa

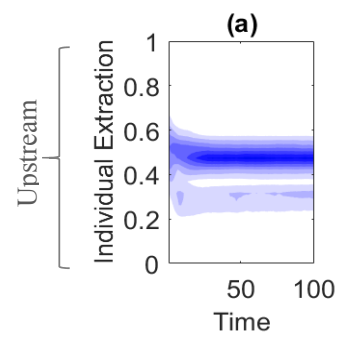

(c)

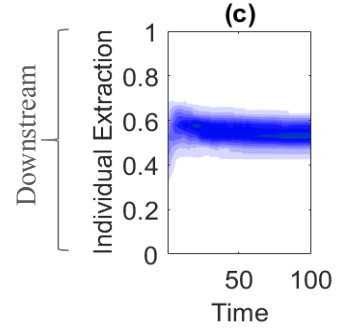

(b)
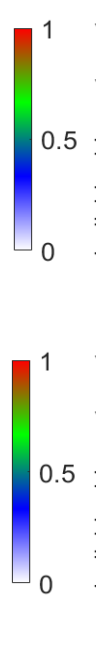

(d)
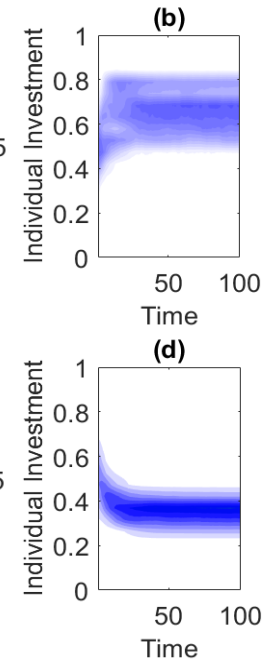

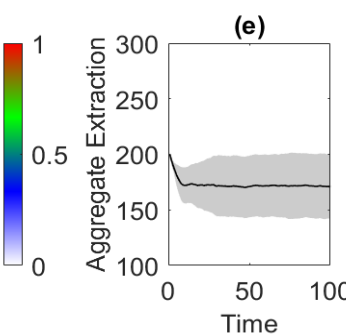

(g)

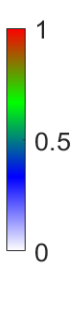

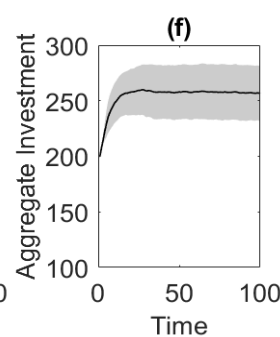

(h)

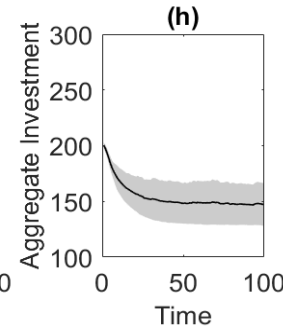

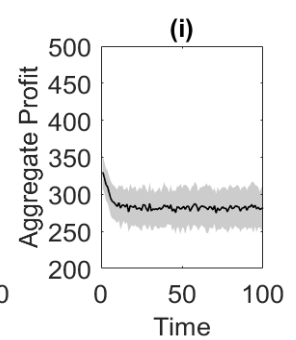

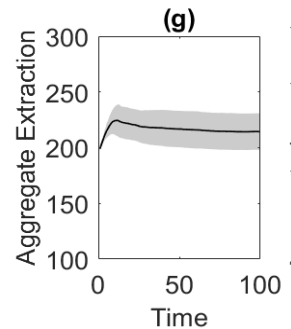

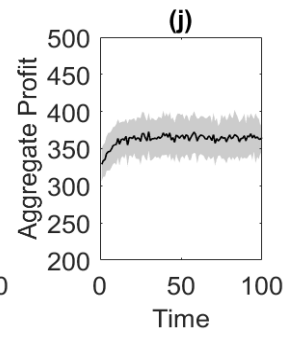

Fig.S2. Effects of social cohesion upstream on investment and extraction efforts when social interactions between communities are absent. The social cohesion coefficients in the upstream is set high $\left(\omega_{1}^{u p}=\right.$ 
2; $\omega_{2}^{u p}=2$ ), while other variables are kept at default values. The top panel represents upstream effort, while the bottom represents the downstream effort. X-axis stands for simulation time. Y-axis represents frequency distribution of extraction and investment efforts among agents at each time step over 50 repeated runs (a-d), and aggregate values averaged over 50 repeated runs (e-j). Colorbar represents the proportion of agents relative to the size of the community.
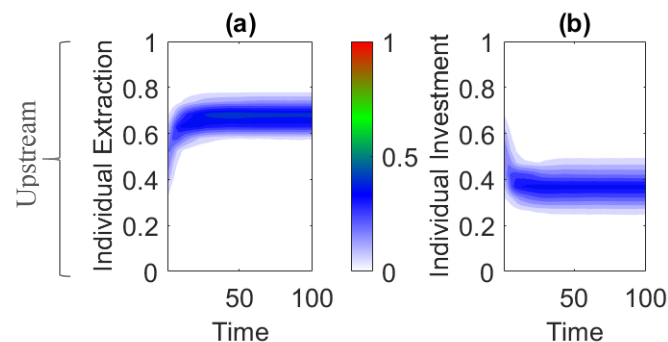

(c)

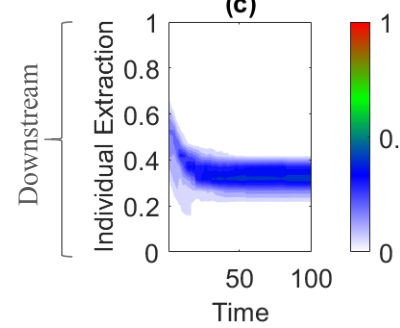

(d)

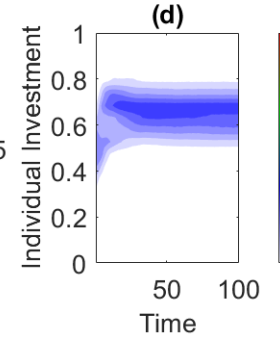

(e)

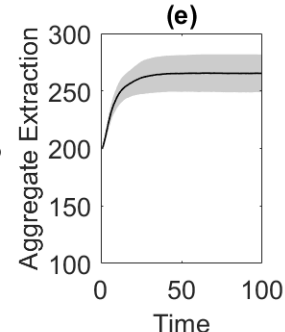

(g)

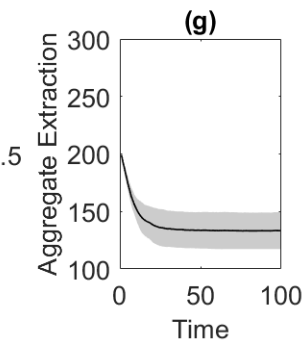

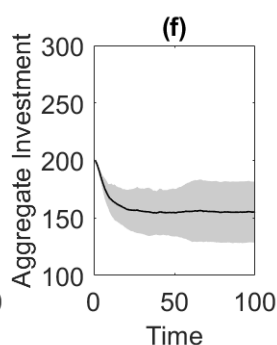

(h)

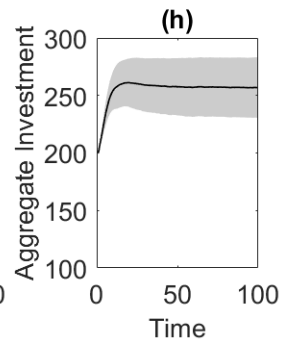

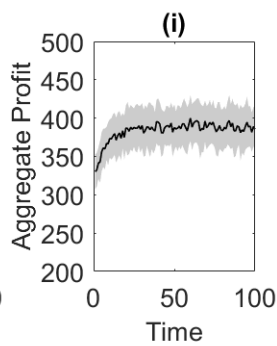

(j)

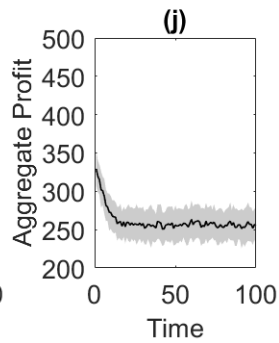

Fig.S3. Effects of social cohesion downstream on investment and extraction efforts when social interactions between communities are absent. The social cohesion coefficients in the downstream is set $\operatorname{high}\left(\omega_{1}^{\text {down }}=2 ; \omega_{2}^{\text {down }}=2\right)$, while other variables are kept at default values. The top panel represents upstream effort, while the bottom represents the downstream effort. X-axis stands for simulation time. $\mathrm{Y}$-axis represents frequency distribution of extraction and investment efforts among agents at each time step over 50 repeated runs (a-d), and aggregate values averaged over 50 repeated runs (e-j). Colorbar represents the proportion of agents relative to the size of the community. 


\title{
Chapter 5-Voting on costly institutions in public goods: Experimental evidence from Cambodia
}

\author{
Tum Nhim ${ }^{1}$, Andries Richter ${ }^{1,2}$, and Esther Schuch ${ }^{1,3}$ \\ ${ }^{1}$ Environmental Economics and Natural Resources group, Wageningen \\ University, The Netherlands \\ ${ }^{2}$ Centre for Ecological and Evolutionary Synthesis, Department of Biosciences, \\ University of Oslo, Norway \\ ${ }^{3}$ Institute for Advanced Sustainability Studies, Potsdam, Germany
}

Nhim, T., Richter, A., \& Schuch, E. (2021). Voting on costly institutions in public goods: Experimental evidence from Cambodia. (under review).

\begin{abstract}
Contributions to public goods are vulnerable to free riding. A potential solution is the implementation of a costly institutional arrangement that deters free riding behavior. In this paper, we analyze to what extent users are willing to bear those costs and vote in favor of costly institutions. We carried out lab-in-the-field experiments Cambodian farmers and fishers in the Kampong Chhnang province. The subjects played public goods games, with an option to vote for a costly institution with a minimum contribution level. In a second experiment, subjects voted between a costless weak enforcement mechanism and a costly strict enforcement mechanism. We found broad support for both costly institutions, and even more so if players had been exposed to resource scarcity in the past. Also, we find that many users support the costly institutions even when the implementation costs outweigh the benefits and the institutions are not necessarily welfare enhancing.
\end{abstract}

Key words: Costly institutions, public goods game, scarcity, field experiment, Cambodia 


\subsection{Introduction}

Resource governance depends largely on cooperation among resource users to organize collective activities (Janssen et al., 2015; Ostrom, 1990; Schill et al., 2016). In many situations, cooperation involves provision of local public goods. For example, farmers have to jointly contribute effort to maintain a shared irrigation system, which is a public good (Ostrom \& Gardner, 1993). The provision of public goods, however, is difficult to achieve because doing so is costly for individuals, but its benefits can be enjoyed by everyone, even if one does not contribute anything. This situation creates tension between individual and collective intereststhe so-called social dilemma, which is a central issue in the management of local and global natural resources (Ostrom et al., 1999). Overcoming social dilemmas can be challenging, especially in the wake of climate change (Bisaro \& Hinkel, 2016). Climate change may alter biophysical conditions of the resource and thus affect the incentive structure of resource appropriation, potentially eroding cooperative arrangements (Nhim et al., 2019; Schlüter et al., 2016). The institutional setting plays an important role in mediating individual and group interests to mitigate risks and impacts of climate change (Agrawal, 2008). A key requirement for successful resource governance-be it formal or informal-is compliance with rules, regulations or norms (Bastakoti \& Shivakoti, 2012; Shreedhar et al., 2019; Tavoni et al., 2012). Without effective enforcement of rules and norms, a self-interested individual has no incentive to follow the prescribed rules and norms because doing so is costly to the individual. A voluntary mechanism to sustain cooperation thus may be at risk due to free riding. This raises the obvious questions, under which conditions users choose to implement appropriate institutional arrangements to safeguard cooperation and how it relates to the cost of institutions (Dannenberg \& Gallier, 2019).

Using lab-in-the-field experiments, this paper aims to understand to what extent resource users are supporting costly institutions if they are designed to discourage free riding when contributing to public goods. Further, we aim to explain what mediating factors affect the probability of subjects supporting such costly institutions. We carried out two experiments, where users can vote between a costly institution - designed to deter free riding - and a costless alternative that opens the door for free riding. First, we consider a mandatory minimum level of contribution to the public good. Such setting deters free riding, but may also crowd out more generous contributions, by providing a clear focal point of contribution. Second, we announce a non-mandatory minimum level of contribution, but users risk to be detected and fined if they 
under-provide. The choice here is to vote between a setting in which detection is probabilistic and a setting in which all free-riders are detected.

The contribution of our paper to the literature is twofold. First, we analyze which factors affect the support for costly institutions among users and how it correlates with observables from the field. In particular, we are interested whether experienced scarcity in the field affects the probability to vote for institutions.

Second, we consider explicitly institutional costs and its impact on welfare. A challenge is that in a standard setup users make decisions how much to contribute after learning the voting outcome, which may revel information about the intentions or cooperative attitude of other players. We obtain a clean measure for welfare by using the strategy-elicitation method, which implies that users decide on each context before learning which one will be relevant.

We find that a significant share of the subjects support costly institutions. Prior experience with resource scarcity increases the likelihood of subjects voting for a costly institution. In particular, we find that the more frequent the subjects are exposed to water scarcity in the past years, the more likely they support costly institutions. We also find that the average contribution in the costly institutions is higher than in the costless institutions, suggesting that the costly institutions do indeed deter free riding without crowding out more generous contributions. While minimum contributions as well as strict enforcement increase average contributions, only the minimum contribution is welfare-enhancing. The increase in cooperation in the costly strict enforcement mechanism is not enough to compensate the institutional cost.

The remainder of this paper is structured as follows. Section 5.2 reviews related literature on endogenous institution in public goods experiment. Section 5.3 describes briefly the context and water management in Cambodia. Section 5.4 describes experimental design and procedures. Section 5.5 presents main experimental results, and section 5.6 concludes.

\subsection{Endogenous institutions and public goods experiments}

Institutions can be categorized in a variety of ways, but it is useful to distinguish formal and informal institutions (North, 1991). In the context of local resource governance, an informal institution is one in which resource users enforce the rule by themselves, e.g. through peer pressure and informal sanctions, while in a formal institution the rule or regulation is enforced 
exogenously or centrally by a third party (Yeboah-Assiamah et al., 2017). Our study features two types of institutions: informal and formal. The informal one is voluntary and monetarily costless, while the formal one has restrictions and is costly.

When it comes to institutional choice in public goods experiments, existing literature has mainly focused on how endogenously chosen and exogenously imposed institutions affect cooperation differently. The effects of both types of institutions on cooperation are mixed. For instance, the contribution to the public goods is higher under a voted institution than under an exogenously imposed institution (Dal Bo et al., 2010; Kamei et al., 2015; Markussen et al., 2014; Sutter et al., 2010; Tyran \& Feld, 2006). In other studies, however, the endogenous choice of institution does not always impact cooperation positively or perform better than the exogenous institutions, especially if users fail to implement appropriate institutions (Gallier, 2020; Kocher et al., 2016; Kroll et al., 2007; Martinsson \& Persson, 2019; Sutter \& Weck-Hannemann, 2003; Vollan et al., 2017).

While previous studies mainly focus on the effects of endogenous versus exogenous institutional choices on cooperation, only more recently the literature explores what determines choice of institutions in public goods experiments (Dannenberg \& Gallier, 2019). The institutional choice is usually made by the subjects through repeated voting (Sutter et al., 2010; Sutter \& Weck-Hannemann, 2003, 2004) or voting once (Kocher et al., 2016), in which the voting outcome is determined by the majority vote (Kroll et al., 2007). In many cases, the contribution decision is made through the direct-response method (Gallier, 2020), while in some cases the strategy method is used (Vollan et al., 2017).

Various papers have explored the role of minimum contribution mechanisms in public goods games, where players can vote between a standard public goods game, which resembles an environment without formal institution, and a public goods game with a minimum contribution level, which resembles an institution that is governed by a tax system (Kocher et al., 2016; Martinsson \& Persson, 2019). Sutter and Weck-Hannemann (2003) and (2004) allow subjects to vote repeatedly whether to implement a binding minimum contribution level in non-linear public goods games. They find that about 80 percent of groups voted for the institution, but the average contribution is not significantly different between groups that support the institution and groups that do not (Sutter \& Weck-Hannemann, 2003). However, asymmetric minimum contribution levels, i.e. group members face different minimum contributions, tend to undermine contributions by those who are disadvantaged. Also, individuals that vote for such 
an institution contribute significantly more in the public goods game than those that do not vote for the institution (Sutter \& Weck-Hannemann, 2004). Kocher et al. (2016) also study the effects of different levels of minimum contribution on cooperation, but in a linear one-shot public goods game. In their study players can vote between a standard public goods game and a public goods games with low minimum contribution (10 percent of the endowment) or high minimum contribution (35 percent of the endowment), both of which are above the Nash equilibrium, but below the social optimum. The institution that is implemented is determined by a random vote, and the contribution decision is made via a strategy elicitation method. Martinsson and Persson (2019) have a similar experimental design, but with a minimum contribution of 25 percent of the endowment. They find that 81 percent of the subjects supported the institution with minimum contribution level and that cooperation is higher in this institution in comparison to the environment without formal institution.

Another strand of literature focuses on endogenous choice of sanctioning institutions in public goods games. In these games, players can vote between a standard public goods game and a public goods game with peer or centralized punishment mechanisms. Feld and Tyran (2002) allow players to vote between a standard public goods game and a public goods game with centralized punishment. Using a strategy method, each player makes a contribution decision for each possible voting outcome, which is determined by the majority rule. Punishment is nondeterrent, i.e. free riding incentive remains, and is executed when the player contributes less than the full endowment. They find that half of the subjects voted for the game with punishment institution. Also, the contribution rate is significantly higher in the game with punishment than in the standard game without punishment, but the payoff is only slightly higher. A similar experiment was conducted by Tyran and Feld (2006), but adding a new feature in which punishment is deterrent, i.e. there is no free riding incentive. They find that more players prefer the institution with deterrent punishment (75\%), as compared to the one with non-deterrent punishment (50\%). Overall, the contributions and payoffs are higher in the institution with both conditions of punishments than in the standard game (Tyran \& Feld, 2006). In a similar setup, Vollan et al. (2017) find that $42 \%$ of players voted for the institution with non-deterrent punishment. Whereas the average contribution is higher in the game with punishment than in the standard game, the payoffs in both institutional settings do not differ. In a similar experiment, but with the direct-response method, Gallier (2017) find that $73 \%$ of players voted for the public goods game with non-deterrent punishment. 
Costs of institutions tend to affect institutional choices and cooperation. For instance, Sutter et al. (2010) let subjects vote between a standard public goods game and a public goods game with costly reward or peer punishment. They find that $62 \%$ of groups voted in favor of the standard public goods game over the alternatives when the reward or punishment is expensive. However, when it is relatively cheap to reward or punish, the standard game becomes much less popular. i.e. receiving only $15 \%$ of votes. The contribution rate is always lower in the standard game than in the game with reward or punishment regardless of voting (Sutter et al., 2010). Dannenberg et al. (2019) let subjects repeatedly vote between a standard public goods game and a public goods game with the option to exclude another member. They find that up to 96 percent of groups vote for the exclusion institution when the institutional cost is absent. When the institutional cost is introduced, however, only 52 percent vote for the institution. Markussen et al. (2014) also let subjects choose between a standard public goods game and the public goods game with a formal or informal punishment scheme. They find that both informal and formal punishment institutions are preferred to the standard public goods game without punishment. Further, the formal punishment institution is the most popular option, especially when it is cheap and deterrent (Markussen et al., 2014). These findings are in line with Kamei et al. (2015).

So far, existing literature on endogenous choice of institution has mainly focused on how various institutional settings such as different levels of minimum contribution or sanctioning strength affect cooperation. The determinants of institutional choices such as those related to personal experience or contextual factors, however, are largely unexplored. Some studies have explored the role of personal characteristics, such as cooperativeness (Ertan et al., 2009), cognitive ability (Dal Bo et al., 2010) or cooperation types (Vollan et al., 2017). After all, most of the studies are based on lab experiments which limits the possibility of exploring the role of personal experiences outside the lab. Such contextual factors are indeed important (Ostrom, 2000, 2009) and can only be tested in the field. Our study analyzes which factors determine the endogenous choice of institutions by including not only variables about individual preferences such as cooperative traits, risk, and trust, but also contextual variables such as wealth and prior experience related to resource conditions.

Previous studies suggest that resource scarcity could have positive or negative effects on cooperation. On the one hand, resource scarcity increases competition and leads to a faster rate of resource depletion (Grossman \& Mendoza, 2003). In addition, past experience about resource scarcity can increase the appropriation rate and thus undermine collective action 
(Blanco et al., 2015; Pfaff et al., 2015). On the other hand, resource scarcity may increase cooperation. For example, concerns for resource scarcity can decrease extraction rate (OsésEraso \& Viladrich-Grau, 2007), either because the scarcity is caused by human or nature (OsésEraso et al., 2008). If the resource users are faced with higher resource uncertainty, they may reduce their harvest rate, as they are concerned about the future decline of the resource (Finkbeiner et al., 2018). To what extent users' experience about scarcity could impact the support of institution, however, has not been studied yet.

\subsection{Water governance in Cambodia}

The governance of local water resources in Cambodia, in particular irrigation water, relies heavily on informal arrangements. Cooperation among farmers in sharing the water and maintaining the irrigation infrastructure thus plays a vital role in facilitating an efficient and fair use of water. However, weak enforcement of rules and social norms that govern water use are obstacles which result in frequent conflicts over water sharing and poor maintenance of water infrastructure (Chea, 2010; Sam \& Pech, 2015). In response to these challenges, Cambodia has been transforming water governance from a centralized system where the state plays a central role in making decisions related to water sharing, water infrastructure maintenance, and rule enforcements, to a more decentralized system where communities have more power in making these decisions (Mak, 2017). This transformation results in a complex, nested system of water governance with multiple actors involved in decision making such as farmers, farmer organizations (Farmer Water User Communities), local government, and the state. In many cases, both the state and communities are involved in the management process which creates a situation where informal arrangements such as social norms and formal written rules can be misaligned and result in conflicts and inefficiency.

Further, water governance in Cambodia is facing other challenges such as increasing water scarcity due to growing demand of water for agriculture and climate change (Mak, 2017; Sam \& Pech, 2015). Climate change is expected to worsen water scarcity (Haddeland et al., 2014; Schewe et al., 2014) and increase water's variability in time and space (Jaeger et al., 2017). In Cambodia, climate change impact is already evident, with changes in rainfall patterns and increased climatic events such as floods and droughts being observed in many areas (Diepart, 2015), posing extra challenges on food security of Cambodian farmers who depend strongly on water resources for farming. 
In the face of such institutional and environmental uncertainties, Cambodia clearly needs an institution that can enhance cooperation and mitigate free riding, especially in the context of climate change (Chem et al., 2010). In response to this, more recently the government is conducting a pilot to test whether the community, through a Famer Water User Community (FWUC), can take over the responsibility to manage the irrigation system by themselves. This kind of organization is a by-law institution that is formed through a participatory process and is responsible for local resource governance. The arrangement is that the members pay the irrigation service fee and this organization is responsible for keeping the irrigation infrastructure in good quality, ensuring provision of irrigation to their members, and enforcing the written rules. Such an institution is indeed a form of formal institution that is voted for by farmers, but to what extent it is supported by the community remains a priori unclear. Understanding which type of institutions would be supported by the community of resource users and whether the institution may enhance cooperation and welfare or not is thus a key policy question for the design and implementation of water institution in Cambodia.

\subsection{Experimental design and procedures}

In our experiment on endogenous institutions, subjects play one-shot public goods games with an option to vote for a preferred institution. We use one-shot games to avoid confounding our results with learning effects. Each game consists of two stages: an institutional choice stage and a voluntary contribution stage. In the first stage, subjects simultaneously and independently choose between two available institutions. In the second stage, each subject interacts in a public goods game with two other random partners. Contributions are made via the strategy-elicitation method. Hence, we ask the participants to make a contribution choice for each institutional setting. The institution that is implemented is determined by the majority of votes in the group they play the public goods game in. By letting people first choose the institution, we get a clear measure on which setting is preferred by the individual. The use of a strategy elicitation allows us to determine whether people behave differently in their preferred institution compared to an institution that is imposed upon them by their peers.

\subsubsection{Experiment 1: A voluntary system versus a costly tax system}

In the first institutional setting, the choice is between a standard public goods game, which resembles an environment without formal institution, that is governed by a voluntary system, and a public goods game with minimum level of contribution, which resembles an institution 
that is governed by a tax system. In the standard public goods game, each subject is endowed with six bills of $1000 \mathrm{KHR}^{2}$, and may contribute any amount between 0 and 6 bills to the public good. The marginal per capita return $(M P C R)$ from the public good is $0.5(\alpha=0.5)$. A rational player would never contribute to the public good since $\alpha<1$. The Nash contribution is thus zero. However, since $\alpha n>1$, where $n=3$ is the group size, it is socially optimal to contribute the entire endowment. This creates a social dilemma situation in our public goods game. Denoting $g$ the amount invested in the public good, the payoff of subject $i$ in the regime of the standard public goods game is given by

$$
\pi_{i}=E_{i}-g_{i}+\alpha\left(g_{i}+g_{-i}\right)
$$

where $E_{i}$ is the endowment, $g_{i}$ the contribution of subject $i$ to the public good and $g_{-i}$ is the contribution by the other two players..

For the public goods game with a minimum contribution level, hereafter referred to as a tax system, the subjects were endowed with 6 bills of KHR and each has to invest at least 3 bills to the public good. By setting the minimum contribution to 3 we ensure that the gain through the mandatory contribution is high enough to off-set the institutional costs. Setting the minimum contribution to 2 would lead to a payoff of 6 per person if everyone just contributes the minimum contribution which is equal to the endowment. Further, by setting the minimum contribution to 3 we are still able to analyze cooperative behavior. Using half the endowment as minimum contribution plays into the observed tendency to contribute around $50 \%$ of the endowment (Ledyard, 1995; Fehr \& Fischbacher, 2003; Burton-Chellew \& West, 2013). ${ }^{3}$ Since $\alpha<1$ and the minimum contribution is 3 bills, the Nash contribution is 3 bills. Like for the standard public goods game, the socially optimal contribution is thus 6 bills, as $\alpha n>1$. Denoting $m$ the cost of institution ( $m=1$ ), the payoff of subject $i$ in the regime of the public goods game with a minimum contribution is given by

$$
\pi_{i}=E_{i}-g_{i}+\alpha\left(g_{i}+g_{-i}\right)-m
$$

with $g \geq 3$.

\footnotetext{
${ }^{2}$ KHR stands for Khmer Riels and 1.00 USD is about 4000 KHR

${ }^{3}$ Ideally, one would vary the minimum contribution level to account for crowding out effects and the natural tendency to contribute half the endowment. Due to time and location constraints we were not able to run those experiments and thus are not able to disentangle these effects.
} 
The Nash equilibrium and socially optimal contributions and the corresponding payoff are given in Table 1. While the Nash contribution in the voluntary system is zero, the Nash contribution in the tax system is 3 . The payoff for the Nash contribution is slightly lower in the voluntary system than in the tax system, but the payoff for the social optimum contribution is higher due to institutional cost in the tax system.

Table 2. Nash and social optimum contributions and corresponding payoffs in voluntary and tax systems

\begin{tabular}{lll}
\hline \hline & Contribution & Payoff \\
\hline \hline Voluntary system & 0.0 & 6.0 \\
Nash & 6.0 & 9.0 \\
Social optimum & & \\
Tax system & 3.0 & 6.5 \\
Nash & 6.0 & 8.0 \\
Social optimum & & \\
\hline
\end{tabular}

\subsubsection{Experiment 2: A weak enforcement versus a strict enforcement system}

In the second institutional setting, the choice is between a weak enforcement and a strict enforcement mechanism. In this setting, there is a rule that each group member must contribute at least 3 bills to the public good. The subjects are free to decide whether to follow the rule or not. However, breaking the rule would get sanctioned with a probability of $0.5(p=0.5) .{ }^{4}$ In our setup, if caught and sanctioned, the subject loses half of his payoff (penalty $\gamma=0.5$ ). ${ }^{5}$ The expected payoff of subject $i$ who violates the rule in the regime of weak enforcement mechanism is given by

$\mathrm{E}\left(\pi_{i}\right)=p\left[E_{i}-g_{i}+\alpha\left(g_{i}+g_{-i}\right)\right]+(1-p) \gamma\left[E_{i}-g_{i}+\alpha\left(g_{i}+g_{-i}\right)\right]$

The payoff of a rule-complying individual is the same as in the standard public goods game (equation 1).

Under the strict enforcement regime, each subject must pay an institutional cost $m$ so every rule breaker is caught and gets sanctioned. In our setup, the cost of operating detection and

\footnotetext{
${ }^{4}$ We set the probability to get caught at $50 \%$ to facilitate understanding by the participants and because we do not have reliable numbers of the likelihood to get caught in similar real life settings in Cambodia.

${ }^{5}$ Again, the main reason to set the penalty at $50 \%$ of the payoff is to facilitate understanding and computation by the participants. Further, losing $50 \%$ of your payoff can be considered high enough to have a deterring effect. While the size of the punishment can have an impact on contribution, we are mainly interested in the institutional choice. Thus, having a punishment that is high enough to have a deterring effect is sufficient.
} 
sanctioning of rule breakers is $m=1$. The payoff of subject $i$ who breaks the rule in the strict enforcement regime is given by

$$
\pi_{i}=\gamma\left[E_{i}-g_{i}+\alpha\left(g_{i}+g_{-i}\right)\right]-m
$$

The payoff of a rule-complying individual is given by equation 2. The Nash equilibrium and social optimum contributions and the corresponding payoff are given in Table 2. From the table, we observe that overall the payoff of the game that features a weak enforcement mechanism is slightly higher than that of the game that features a strict enforcement mechanism, due to the institutional cost incurred in the latter. In both games, the Nash contribution is to follow the rule and contribute 3 bills.

Table 3. Nash and social optimum contributions and corresponding payoffs in weak and strict enforcement mechanisms

\begin{tabular}{ccc}
\hline \hline & Contribution & Payoff \\
\hline \hline Weak enforcement & 3.0 & 7.5 \\
Nash & 6.0 & 9.0 \\
Social optimum & & \\
Strict enforcement & 3.0 & 6.5 \\
Nash & 6.0 & 8.0 \\
Social optimum & & \\
\hline
\end{tabular}

Along with the public goods games with both costly institutional settings, we also run a GneezyPotter risk-elicitation game (Gneezy \& Potters, 1997) ${ }^{6}$, to characterize the risk preferences of participants, followed by a standard public goods game and a public goods game with strategy elicitation, to study their cooperative types. After the games, we also conducted structured interviews with each participant. We asked question along five different topics: (i) community trust, support, and collective action, (ii) scarcity, (iii) shocks, (iv) institutional settings experienced, and (v) demographics. Details on the survey and exact methods of eliciting risk and social preferences are given in Schuch et al. (2021). The study was reviewed by the Social Sciences Ethic Committee of Wageningen University and registered as a pre-analysis plan; see Richter et al. (2020).

\footnotetext{
${ }^{6}$ We use a Gneezy-Potter risk elicitation game since it is easy to understand for the participants and is straight forward to implement in a field setting with a sample pool where illiteracy is high.
} 


\subsubsection{Implementation}

Our study sample consists of rural Cambodian farmers from 21 villages in the Kampong Chhnang province (Figure 1). We focus on three communes, namely Kouk Banteay (8 out of 8 villages), Taing Krasaing (we randomly drew 6 out of 12 villages), and Tuol Khpous (7 out of 7 villages). While farming is prevalent in all these communes, fishing dependency and access to irrigation water differs. Thus, only some farmers have the option to use fishing as a secondary income. Fishing dependency is relatively high in Kouk Banteay, and low in Tuol Khpous and Tank Krasaing. Further, Kouk Banteay has more access to water than the other two as it has more canal systems.

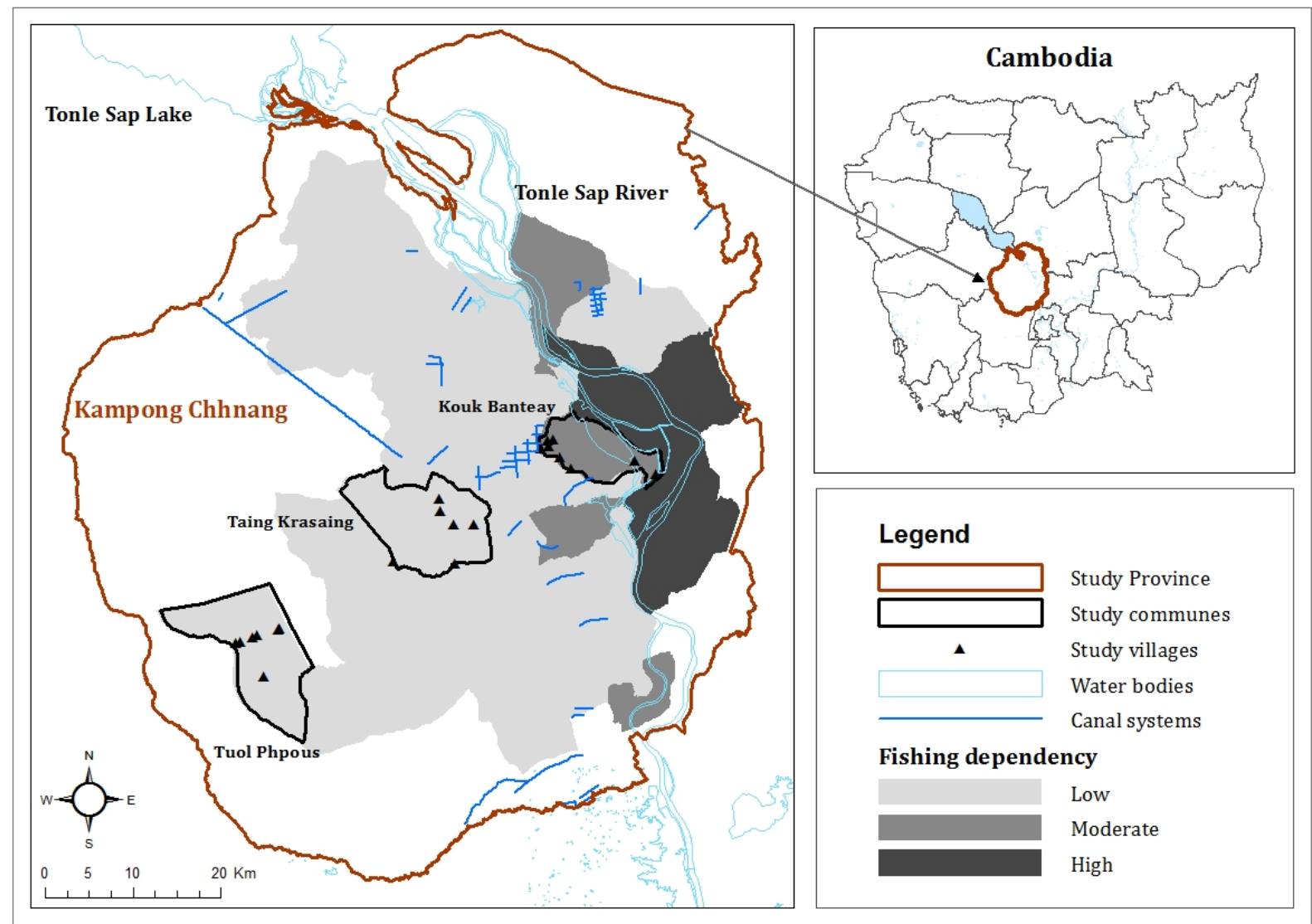

Fig. 1. Map of study area in Kampong Chhnang province, Cambodia

The participants were recruited via village chiefs. We informed village chiefs about the criteria for recruitment. We required that the participants should be (i) 18 years or older, (ii) only one person per household (preferably the household head or spouse), (iii) healthy as the experiments would take around 3 hours, and (iv) from all social sphere within the village. Thus, our sample is representative of the area but not of Cambodian farmers. At the beginning of each session the village chiefs where there to greet people and ensure that everything is running smoothly before 
they left for their respective work. This way we could ensure that the village chiefs did not influence the decision-making.

All experiments were pen-and-paper experiments with verbal instructions and posters and examples to aid understanding. The exact instructions of the experiments and the materials used are given in the Online Appendix. For each of the games the individuals were assigned randomly to new groups. There was no feedback given between the experiments. To account for the time investment we pay each participant a show-up fee of 4000 KHR and another 6000 KHR if they stay till the end in addition to the money earned in one of the games. At the end of a session one of the games was chosen randomly for each participant to avoid confounding effects between the games. The average payout is $17000 \mathrm{KHR}$ which corresponds to $\$ 4.25$ and provides a sufficient incentive to participate given the average daily wage in the region of $\$ 4.50$. The order of the games played is the same for every individual. Thus, we cannot rule out that order effects are confounding our results.

In all experiments the participants were asked to divide their endowment of $6000 \mathrm{KHR}$ between a group fund (a green envelope) and a private fund (a white envelope). The only difference was in the tax treatment. Here, the participants were given only 3000 KHR to divide between the private and the public fund while already $3000 \mathrm{KHR}$ were in the public fund envelope. This way we made it salient that 3000 KHR are going towards the public fund (the mandatory tax) and the participants only had a distributional choice in regard to the other $3000 \mathrm{KHR}$. In the punishment treatments there is only the rule to contribute 3000 but the participants have a choice whether they want to follow this rule, thus we provide them with $6000 \mathrm{KHR}$.

\subsection{Results}

\subsubsection{Data description}

Out of the 315 subjects, 13 were dropped from the dataset due to redundant/missing identities in the survey, limited time to take part in the survey or the experiments. In the final dataset, we thus have 302 observations for the experimental tasks and 282 observations for both the experiment and the survey. Our sample has more female (63\%) than male (37\%) participants. The average age is about 46 years, and no one is below 18 years old. Most of them have very low education (average schooling years of 4). On average, a household has about 5 members. Furthermore, most of the subjects are farmers as primary occupation. 
To measure cooperative traits, we asked participants to play conditional public goods games to understand their prosocial preferences. In the game, subjects were endowed with 6 bills of 1000 KHR and asked to make seven decision rounds on how much to contribute to the public good, knowing what the partner contributes. Using the hierarchical cluster analysis (Fallucchi et al., 2018), the subjects can be divided into five groups in terms of their prosocial preferences (Table 3); see also Schuch et al. (2021). Low, medium, and high unconditional cooperators are those who made low (mean of 1.77), moderate (mean of 2.54), and high (mean of 4.44) contributions respectively regardless of what the partner contributed. Conditional cooperators are the ones who try to match the contribution of partners (mean of 3.16). Finally, we have some subjects (79 out of 302) whose contribution level seems to be irregular, referred to as "other behavioral type". In terms of individual preferences, we measured risk aversion on a scale 0 to 6 , with zero being not at all risk averse and 6 being highly risk averse. In our study sample, overall, the subjects are fairly risk averse.

In our survey we measured trust level by asking them how many out of 10 villagers would voluntarily join a collective project in the village if invited. The average trust level is around 7.0. We also measured wealth of each household. First, we asked whether the household owns a motorboat since fishery is an important source of income in the study area. As a result, only $9 \%$ of them own a motorboat, which is considered wealthier than those who do not. Further, $22 \%$ of the respondents own farmland for growing other crops rather than rice. When it comes to assets related to rice farming, which is the most important livelihood option in the area, $46 \%$ of them own paddy land close to an irrigable water source, which is within the distance of 100 meters. Remittance from migration is another important source of income, and in our study sample $32 \%$ of them did receive remittance in the past year.

Finally, we also elaborate on household experience about water scarcity. Firstly, we asked how many times the household experienced irrigation water scarcity in the past 5 years. The average frequency of water scarcity experienced in the past 5 years is 0.95 . Further, $62 \%$ of the respondents did not have enough water for irrigation in the past year. With regard to those who have fishing experience, $19 \%$ of them have experienced catch decline in the past year, and $12 \%$ reported that they experienced stock collapse. Lastly, $57 \%$ of the respondents also reported that they experienced income shock in the past year. 
Table 4. Descriptive statistics of relevant variables

\begin{tabular}{lrrrrr}
\hline \hline Variable & Obs. & Mean & Std. Dev. & Min. & Max. \\
\hline \hline Socioeconomics & & & & & \\
Gender (male=1) & 281 & 0.37 & 0.48 & 0.00 & 1.00 \\
Age & 282 & 46.20 & 13.22 & 18.00 & 80.00 \\
Schooling years & 282 & 4.05 & 3.48 & 0.00 & 16.00 \\
Number of household members & 282 & 4.76 & 1.70 & 1.00 & 11.00 \\
Individual preferences & & & & & \\
Risk aversion & 282 & 3.18 & 1.00 & 0.00 & 6.00 \\
Trust & 282 & 7.33 & 2.35 & 0.00 & 10.00 \\
Wealth & & & & & \\
Owning a motorboat & 282 & 0.09 & 0.28 & 0.00 & 1.00 \\
Owning farmland for growing other crops & 282 & 0.22 & 0.42 & 0.00 & 1.00 \\
Owning paddy land close to water source & 282 & 0.46 & 0.50 & 0.00 & 1.00 \\
Receiving income from remittance & 282 & 0.32 & 0.47 & 0.00 & 1.00 \\
Experience about scarcity & & & & & \\
Frequency of water scarcity experienced & 282 & 0.95 & 2.93 & 0.00 & 20.00 \\
Having enough water for irrigation & 282 & 0.62 & 0.49 & 0.00 & 1.00 \\
Having experienced catch decline & 282 & 0.19 & 0.39 & 0.00 & 1.00 \\
Having experienced stock collapse & 282 & 0.12 & 0.33 & 0.00 & 1.00 \\
Having experienced income shock & 282 & 0.57 & 0.50 & 0.00 & 1.00 \\
\hline
\end{tabular}

\subsubsection{Voting and contribution in costly institutions}

\section{Voting on costly institutions}

Our experiment features two types of institutional settings. In the first setting, the choice of institution is between a costless voluntary system where there is no rule prescribing how much each group member has to contribute to the public good, and a costly tax system where each group member has to contribute at least half of the endowment to the public good. In the second setting, the institutional choice is between a costless weak enforcement mechanism and a costly strict enforcement mechanism. Under the weak enforcement mechanism, rule breakers (those who contribute less than half of the endowment) have a $50 \%$ chance of escape from punishment. Under the strict enforcement mechanism, however, every rule breaker is punished. 
Results of voting between a voluntary system and a tax system show that a significant share of subjects $(62.25 \%)$ voted for the tax system, and $63.55 \%$ of the groups ended up having this institution implemented. Similarly, the majority of the subjects $(61.59 \%)$ voted for a strict enforcement mechanism, and $62.50 \%$ of the groups ended up having the strict enforcement mechanism implemented. On the other hand, if we look at voting behavior across institutions (Fig. 2), we observe that $23.84 \%$ of the subjects voted for both costless institutions, i.e. the voluntary system and the weak enforcement mechanism, and up to $47.68 \%$ voted for both costly institutions: the tax system and the strict enforcement mechanism. $14.57 \%$ of them voted for the costly tax system and the weak enforcement mechanism, while the rest (13.91\%) voted for the voluntary system and the costly strict enforcement mechanism.

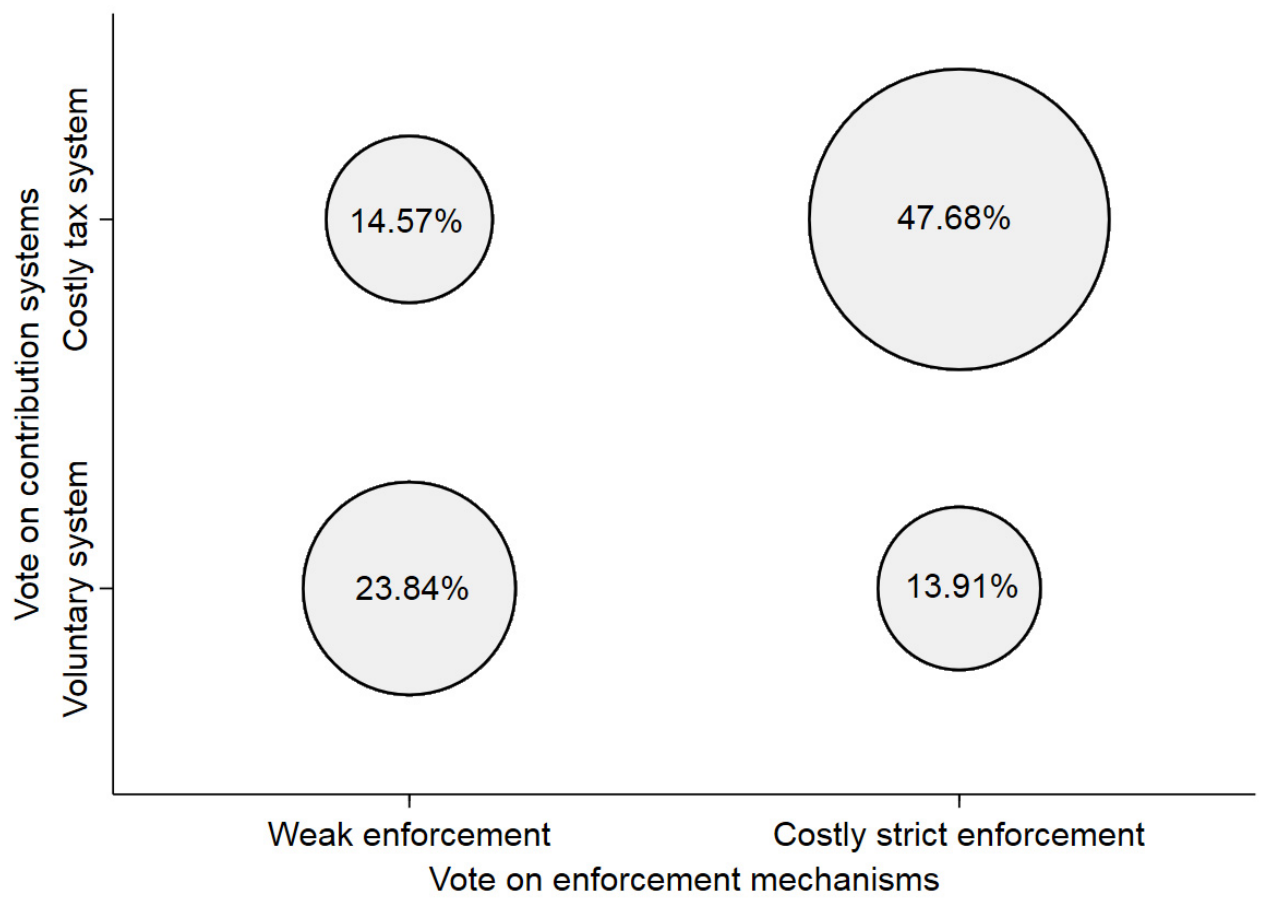

Fig. 2. Percentage of votes across institutions

\section{Contributions in costly institutions}

Here, we want to understand cooperative behavior of subjects in terms of their contribution to the public good in both institutional settings (Figure 3). Overall, only a very small proportion of subjects contribute nothing to the public good. Also, contributing the minimum requirement (3 bills) - implemented or not - to the public good is the most popular option across institutions. The most frequently observed contribution level in the standard voluntary contribution setting is 3 , so half of the endowment. This effect is well documented in the literature and our subject pool is no exception. What is interesting is that we see an increase in contributions if the tax/rule 
is set to 3. Instead of an anchoring effect on 3 we rather see that people opt to be more cooperative than is required. We also see that people hardly contribute 0 , even if given the choice and no punishment. This is in line with the cooperative traits we determined. In the first setting (Fig. 3a), we observe that many subjects still contribute more than the required minimum level in the tax system, indicating that the tax system does not crowd out the voluntary contribution beyond the minimum level. In the second setting (Fig. 3b), the subjects largely follow the rule (contributing 3 bills or more) under both weak and strict enforcement mechanisms. Under the strict enforcement mechanism, however, a very small proportion of them contribute less than 3 bills, even if that means they surely get punished.

Paired-sample t-tests were performed to determine if there are differences in contribution between the costless and costly institutions. Overall, the contribution level in the costly institutions is significantly higher than that in the costless institutions. In the first setting, the mean contribution level is higher under the tax system $(4.02 \pm 0.97)$, as compared to the voluntary system $(2.92 \pm 1.16)$, a statistically significant difference of $1.10(t=13.458, p=0.0000)$. In this setting, the percentage of players contributing the social optimum level (6 bills) is higher in the tax system (about 10\%) than in the voluntary system (about 3\%). In the second setting, the mean contribution level is higher under the strict enforcement mechanism $(3.66 \pm 0.91)$ than under the weak enforcement mechanism $(3.44 \pm 0.85)$, a statistically significant difference of $0.21(t=3.898, p=0.0001)$.

(a)

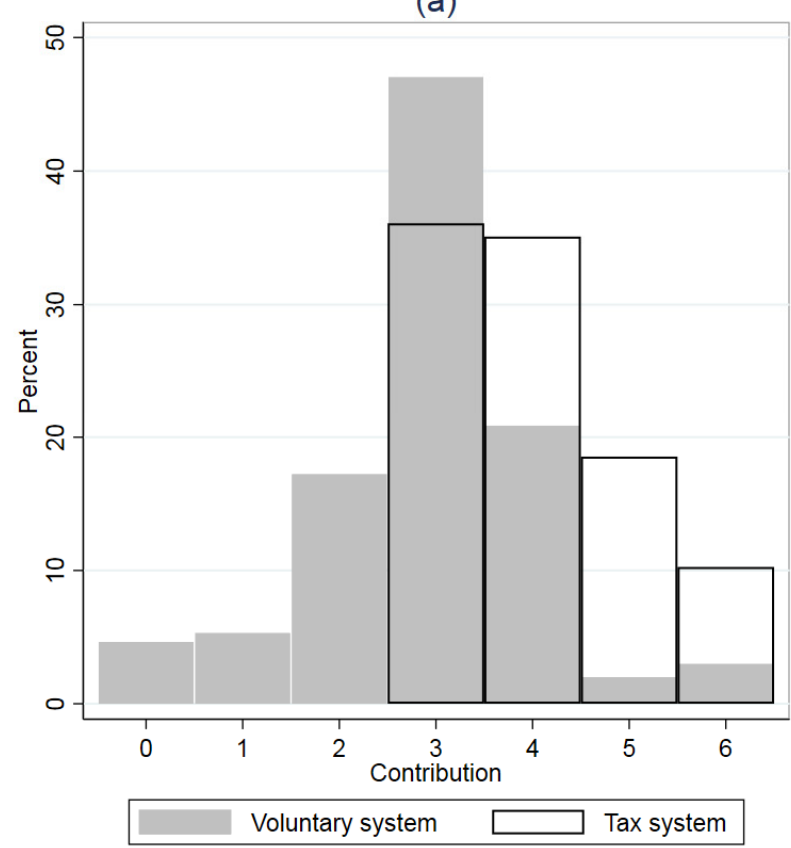

(b)

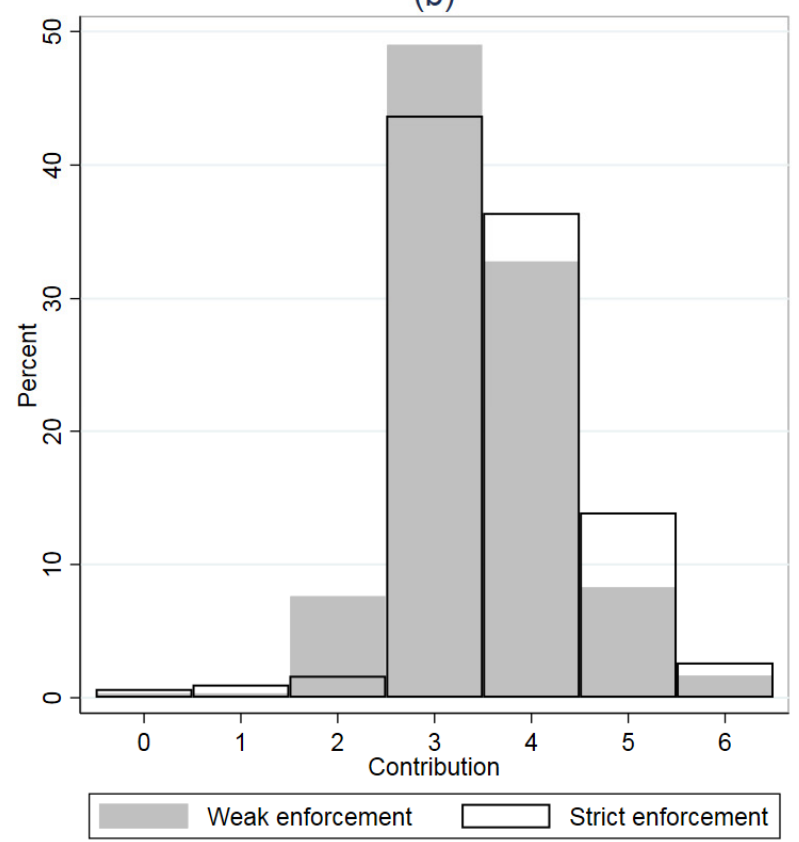

Fig.3. Distribution of contribution levels between costless and costly institutions 


\subsubsection{Individual payoff of each institution}

Figure 4 shows individual payoff conditional on the institutional regime. It is worth to recall that the contribution decision in our game is based on the strategy method, meaning that each subject decides on the contribution in each game, without having known the voting outcome. Therefore, we are able to compare the payoff levels between the two institutional regimes. As for the first institutional setting (Fig. 4a), we observe higher payoff under the tax system than in the voluntary system, suggesting that there is a welfare improvement in the costly institution, as compared to the costless institution. In contrast, we see lower payoff under the strict enforcement mechanism, as compared to the weak enforcement mechanism (Fig. 4b). The reason is simply that the cost of the strict enforcement mechanism does not outweigh the benefit in terms of higher cooperation.

(a)

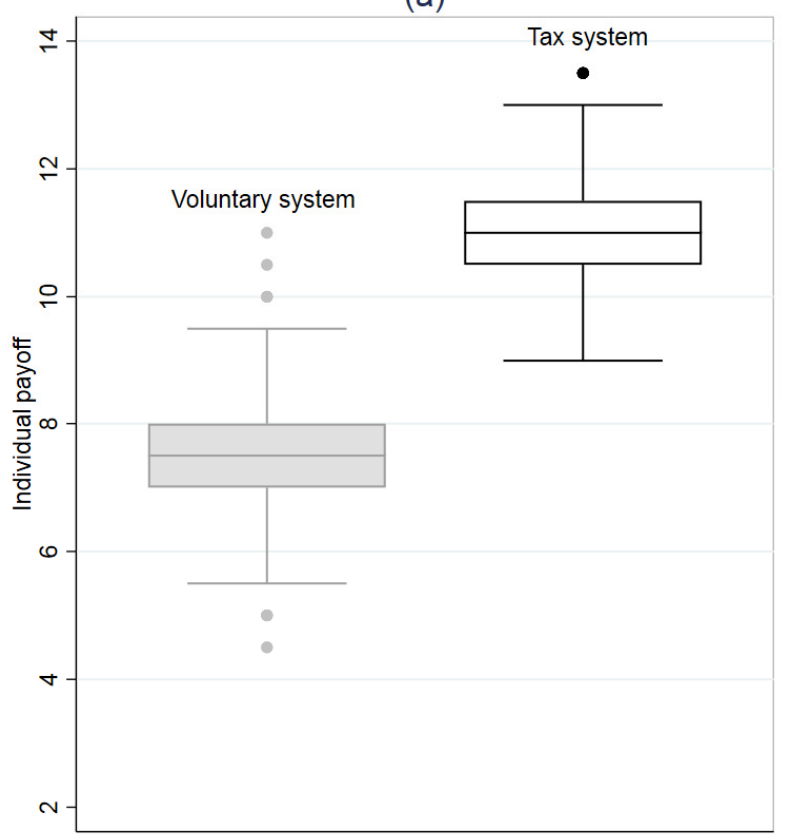

(b)

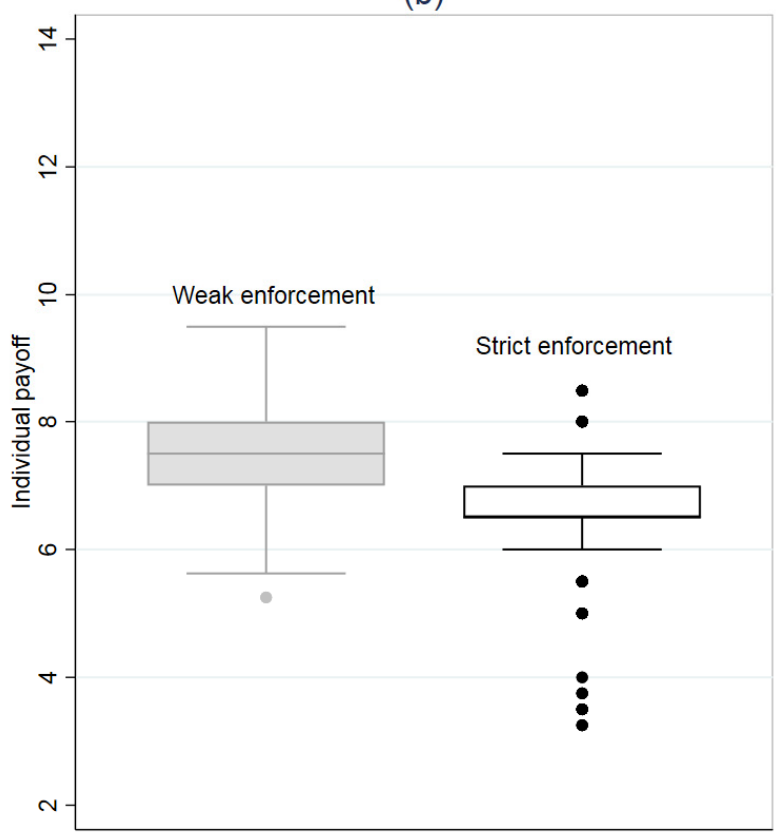

Fig. 4. Boxplot showing individual payoffs for the different institutional settings. The horizontal line inside the rectangle represents the median payoff and the ones either side of the rectangle show lower and upper quartiles. Circle markers are outlier.

\subsubsection{Determinants of institutional choice}

Logistic regressions were run to understand effects of prior experience about scarcity, wealth, and individual characteristics on the likelihood of subjects voting for a costly tax system (Table 4). In the first model, we observe that having experienced scarcity impacts subjects' voting behavior. In particular, the more exposed to water scarcity people have been in the past, the 
more likely they are to vote for the tax system. This result holds true even when we control for village effects (model 2). If we distinguish between fishers (model 3) and non-fishers (model 4), the positive effect of exposure to water scarcity on the support of the tax system disappears among non-fishers. It is worth to note that fishers also do farming, and when we asked participants in the survey to identify their primary occupation, 52 out of 63 fishers responded that rice farming is their primary occupation, and only one of them considered himself as a fisher. It seems to point to the fact that fishing is more like an alternative livelihood strategy, which is used to cope with water scarcity. Further, 44 out of 63 fishers (70\%) also cultivate dry season rice, which is a relative water-consuming farming system, suggesting that water scarcity is highly relevant for those who also engage in fishing. We also look at other variables that describe household experience of scarcity such as whether the household has had enough water for irrigation, experienced catch decline and fish stock collapse, but we do not find any significant effects on their voting behavior. In addition, we also observe that household experience of income shocks in the past years does not impact voting behavior either.

Wealth, however, can have different effects on voting behavior, depending on whether it is related to rice farming or not. For instance, the subjects who own paddy land close to irrigable water sources are less likely to vote for the tax system. The reason could be that this group of players has higher capacity to cope with water scarcity as well as more control over water access, finding it less important to have such an institution. We find that those who receive offfarm income from remittance are more likely to vote for the tax system. We also included other variables related to wealth such as whether the households own a motorboat or whether they own farmland for growing other crops rather than rice, but we find no significant effects. In terms of individual preferences such as risk and trust level, we do not find any significant effects on their voting behavior. We also control for village effects, as indicated in the second model, but we find no major differences.

Similarly, we run logistic regressions to understand the effects of prior experience about scarcity, wealth, and individual preferences on the probability that the subjects vote for the strict enforcement mechanism (Table 5). Overall, we find that more exposure to water scarcity increases the likelihood of subjects' voting for the strict enforcement mechanism. Again, this effect is more present among those who are also fishing. Other variables related to experience about scarcity, such as whether the household has had enough water for irrigation and experienced catch decline and fish stock collapse, have no significant effects on the voting 
behavior. Whether the household experienced income shock in the past year does not impact voting behavior either.

We do find that wealth affects voting behavior. While the subjects who own a motorboat are less likely to vote for the strict enforcement mechanism, those who own a farmland for growing other crops rather than rice are more likely to vote for the strict enforcement mechanism. Overall, other variables related to wealth such as whether households owns paddy land close to water source and whether they receive income from remittance have no significant effects on the likelihood of subjects voting for the strict enforcement mechanism. However, among fishers the effects of wealth are largely negative. Finally, if we look at the effects of individual preferences such as risk aversion and trust on the voting behavior, we observe that risk averse fishers are more likely to vote for the strict enforcement mechanism.

Table 5. Effects of experience about scarcity, wealth and individual preferences on subject's voting for the tax system

\begin{tabular}{|c|c|c|c|c|}
\hline \multirow[t]{2}{*}{ Dep. Var.: Voting for tax system $(1 / 0)$} & \multicolumn{2}{|c|}{ Pooled } & \multirow{2}{*}{$\begin{array}{c}\text { Fishers } \\
(3)\end{array}$} & \multirow{2}{*}{$\begin{array}{c}\text { Non-fishers } \\
(4)\end{array}$} \\
\hline & (1) & (2) & & \\
\hline \multicolumn{5}{|l|}{ Experience about scarcity } \\
\hline Frequency of water scarcity experienced & $0.098^{*}(0.053)$ & $0.111^{* *}(0.056)$ & $0.575^{* *}(0.242)$ & $0.053(0.055)$ \\
\hline Having enough water for irrigation & $0.599(0.368)$ & $0.707^{*}(0.411)$ & $1.602(1.302)$ & $0.693(0.428)$ \\
\hline Having experienced catch decline & $-0.522(0.576)$ & $\left.-1.138^{*} 0.670\right)$ & $-1.367(2.107)$ & \\
\hline Having experienced stock collapse & $0.600(0.894)$ & $0.910(0.895)$ & $0.804(1.284)$ & \\
\hline Having experienced income shock & $-0.103(0.223)$ & $0.0005(0.327)$ & $2.152(1.441)$ & $-0.271(0.282)$ \\
\hline \multicolumn{5}{|l|}{ Wealth } \\
\hline Owning a motorboat & $-0.0247(0.851)$ & $0.557(0.989)$ & $0.155(1.609)$ & \\
\hline Owning farmland for growing other crops & $0.626(0.446)$ & $0.316(0.470)$ & $0.137(2.328)$ & $0.834^{*}(0.489)$ \\
\hline Owning paddy land close to water source & $-0.849^{* * *}(0.296)$ & $-0.932^{* *}(0.375)$ & $-2.428^{*}(1.408)$ & $-0.798^{* *}(0.331)$ \\
\hline Receiving income from remittance & $0.647^{* *}(0.321)$ & $0.394(0.357)$ & $2.886^{*}(1.680)$ & $0.579(0.408)$ \\
\hline \multicolumn{5}{|l|}{ Individual preferences } \\
\hline Risk aversion & $0.054(0.159)$ & $0.151(0.177)$ & $0.237(1.059)$ & $0.066(0.171)$ \\
\hline Trust & $0.014(0.060)$ & $0.044(0.072)$ & $0.041(0.174)$ & $0.010(0.068)$ \\
\hline Constant & $-1.001(0.823)$ & $1.230(1.811)$ & $-10.39^{*}(5.797)$ & $-0.664(0.858)$ \\
\hline Commune fixed effects & $\sqrt{ }$ & & $\sqrt{1}$ & $\sqrt{ }$ \\
\hline Village fixed effects & & $\sqrt{ }$ & & \\
\hline Cooperative traits & $\sqrt{ }$ & $\sqrt{ }$ & $\sqrt{ }$ & $\sqrt{ }$ \\
\hline Socio-demographics & $\sqrt{ }$ & $\sqrt{ }$ & $\sqrt{ }$ & $\sqrt{ }$ \\
\hline Observations & 281 & 267 & 63 & 218 \\
\hline Pseudo $R^{2}$ & 0.156 & 0.221 & 0.388 & 0.159 \\
\hline
\end{tabular}

Standard errors in parentheses

${ }^{*} p<.10,{ }^{* *} p<.05,{ }^{* * *} p<.01$ 
Table 6. Effects of experience about scarcity, wealth and individual preferences on subject's voting for a strict enforcement

\begin{tabular}{|c|c|c|c|c|}
\hline \multirow[t]{2}{*}{ Dep. Var.: Voting for strict enforcement $(1 / 0)$} & \multicolumn{2}{|c|}{ Pooled } & \multirow{2}{*}{$\begin{array}{c}\text { Fishers } \\
(3)\end{array}$} & \multirow{2}{*}{$\begin{array}{c}\text { Non-fishers } \\
(4)\end{array}$} \\
\hline & (1) & (2) & & \\
\hline \multicolumn{5}{|l|}{ Experience about scarcity } \\
\hline Frequency of water scarcity experienced & $0.142^{* *}(0.072)$ & $0.157^{* *}(0.069)$ & $0.391^{*}(0.221)$ & $0.096(0.071)$ \\
\hline Having enough water for irrigation & $0.329(0.359)$ & $0.607(0.414)$ & $0.588(1.636)$ & $0.281(0.353)$ \\
\hline Having experienced catch decline & $0.318(0.623)$ & $-0.084(0.658)$ & $-0.369(1.370)$ & \\
\hline Having experienced stock collapse & $0.340(0.687)$ & $0.725(0.778)$ & $0.603(1.469)$ & \\
\hline Having experienced income shock & $0.005(0.223)$ & $0.079(0.311)$ & $-0.344(0.891)$ & $0.061(0.250)$ \\
\hline \multicolumn{5}{|l|}{ Wealth } \\
\hline Owning a motorboat & $-1.800^{* *}(0.829)$ & $-1.350^{*}(0.731)$ & $-2.138^{* *}(0.970)$ & \\
\hline Owning farmland for growing other crops & $0.901^{*}(0.541)$ & $0.578(0.402)$ & $-1.763^{*}(1.055)$ & $1.079(0.661)$ \\
\hline Owning paddy land close to water source & $-0.220(0.361)$ & $-0.009(0.374)$ & $-2.850^{* * *}(0.934)$ & $-0.013(0.391)$ \\
\hline Receiving income from remittance & $0.377(0.306)$ & $0.377(0.347)$ & $-1.170(0.802)$ & $0.437(0.372)$ \\
\hline \multicolumn{5}{|l|}{ Individual preferences } \\
\hline Risk aversion & $0.0291(0.201)$ & $0.201(0.178)$ & $1.726^{* *}(0.847)$ & $-0.0273(0.228)$ \\
\hline Trust & $0.055(0.072)$ & $0.082(0.073)$ & $0.216(0.161)$ & $0.027(0.078)$ \\
\hline Constant & $-0.873(0.989)$ & $-1.562(1.405)$ & $-4.970(4.002)$ & $-0.502(1.193)$ \\
\hline Commune fixed effects & $\sqrt{ }$ & & $\sqrt{ }$ & $\sqrt{ }$ \\
\hline Village fixed effects & & $\sqrt{ }$ & & \\
\hline Cooperative traits & $\sqrt{ }$ & $\sqrt{ }$ & $\sqrt{ }$ & $\sqrt{ }$ \\
\hline Socio-demographics & $\sqrt{ }$ & $\sqrt{ }$ & $\sqrt{ }$ & $\sqrt{ }$ \\
\hline Observations & 281 & 264 & 63 & 218 \\
\hline Pseudo $R^{2}$ & 0.113 & 0.198 & 0.429 & 0.133 \\
\hline
\end{tabular}

Standard errors in parentheses

${ }^{*} p<.10,{ }^{* *} p<.05,{ }^{* * *} p<.01$

\subsubsection{Contributions in costless and costly institutions}

Table 6 shows that contributions to the public fund in both voluntary and tax systems do not depend on whether the subjects have voted for the institution. We also analyze how the cooperative traits interact with the institutional regimes. Not unexpected, we find is that low unconditional cooperators contribute significantly less in all institutional regimes than the other groups. We also looked at the difference in contributions between the two institutions that could be voted for (models $3 \& 6$ ) to see whether certain cooperative types contribute more (or less) under the tax regime, but we do not observe this effect.

Similarly, we also analyze how contributions to the public good in the weak and strict enforcement mechanisms differ (Table 7). Again, the contributions in both institutions do not depend on whether the subjects have voted for the institution. We find that low and moderate unconditional cooperators contribute significantly less in both institutions, as compared to the 
high unconditional cooperators. Again, we find no significant difference in contributions in both institutions, when controlling for cooperative types (models $3 \& 6$ ).

Table 7. Factors explaining contribution behavior in the voluntary and tax systems

\begin{tabular}{|c|c|c|c|c|c|c|}
\hline "Dep. Var.: Contribution for (1), (2), (4), \& (5) & (1) & (2) & (3) & (4) & (5) & (6) \\
\hline Dep. Var.: Difference in contributions for (3) & Voluntary & Tax & Tax- & Voluntary & Tax & Tax- \\
\hline$\&(6)$ & & & Voluntary & & & Voluntary \\
\hline \multirow[t]{2}{*}{ Having voted for tax system } & 0.234 & -0.0361 & -0.270 & 0.162 & 0.008 & $\begin{array}{c}-0.154 \\
\end{array}$ \\
\hline & $(0.178)$ & $(0.140)$ & $(0.208)$ & $(0.165)$ & $(0.131)$ & $(0.195)$ \\
\hline \multicolumn{7}{|l|}{ Cooperative traits } \\
\hline \multirow[t]{2}{*}{ Other } & 0.077 & 0.022 & -0.055 & 0.005 & 0.010 & 0.004 \\
\hline & $(0.221)$ & $(0.210)$ & $(0.291)$ & $(0.191)$ & $(0.174)$ & $(0.234)$ \\
\hline \multirow[t]{2}{*}{ Conditional Cooperators } & -0.250 & -0.163 & 0.0870 & -0.320 & -0.147 & 0.173 \\
\hline & $(0.232)$ & $(0.233)$ & $(0.311)$ & $(0.214)$ & $(0.181)$ & $(0.242)$ \\
\hline \multirow[t]{2}{*}{ Low Unconditional Cooperators } & -0.518 & $-0.550^{* *}$ & -0.032 & $-0.489^{*}$ & $-0.601^{* * *}$ & -0.112 \\
\hline & $(0.345)$ & $(0.243)$ & $(0.392)$ & $(0.260)$ & $(0.193)$ & $(0.298)$ \\
\hline \multirow[t]{2}{*}{ Medium Unconditional Cooperators } & 0.008 & -0.205 & -0.213 & -0.063 & -0.215 & -0.152 \\
\hline & $(0.232)$ & $(0.252)$ & $(0.372)$ & $(0.244)$ & $(0.199)$ & $(0.313)$ \\
\hline \multirow[t]{2}{*}{ Constant } & $4.158^{* * *}$ & $4.609^{* * *}$ & 0.451 & $4.868^{* * *}$ & $4.385^{* * *}$ & -0.483 \\
\hline & $(0.507)$ & $(0.404)$ & $(0.519)$ & $(0.498)$ & $(0.462)$ & $(0.589)$ \\
\hline Commune fixed effects & $\sqrt{ }$ & $\sqrt{ }$ & $\sqrt{ }$ & & & \\
\hline Village fixed effects & & & & $\sqrt{ }$ & $\sqrt{ }$ & $\sqrt{ }$ \\
\hline Risk aversion & $\sqrt{ }$ & $\sqrt{ }$ & $\sqrt{ }$ & $\sqrt{ }$ & $\sqrt{ }$ & $\sqrt{ }$ \\
\hline Socio-demographics & $\sqrt{ }$ & $\sqrt{ }$ & $\sqrt{ }$ & $\sqrt{ }$ & $\sqrt{ }$ & $\sqrt{ }$ \\
\hline Observations & 281 & 281 & 281 & 281 & 281 & 281 \\
\hline$R^{2}$ & 0.100 & 0.120 & 0.034 & 0.214 & 0.211 & 0.169 \\
\hline Adjusted $R^{2}$ & 0.060 & 0.081 & -0.009 & 0.120 & 0.116 & 0.070 \\
\hline
\end{tabular}

Standard errors in parentheses

${ }^{*} p<.10,{ }^{* *} p<.05,{ }^{* * *} p<.01$ 
Voting on costly institutions in public goods

Table 8. Factors explaining contribution behavior in the weak and strict enforcement mechanisms

\begin{tabular}{|c|c|c|c|c|c|c|}
\hline Dep. Var.: Contribution for (1), (2), (4), \& (5) & (1) & (2) & (3) & (4) & (5) & (6) \\
\hline Dep. Var.: Difference in contributions for (3) & Weak & Strict & Strict- & Weak & Strict & Strict-weak \\
\hline$\&(6)$ & & & Weak & & & \\
\hline \multirow[t]{2}{*}{ Having voted for strict enforcement } & 0.0368 & -0.0027 & -0.0395 & 0.0362 & -0.0962 & -0.132 \\
\hline & $(0.141)$ & $(0.114)$ & $(0.169)$ & $(0.118)$ & $(0.113)$ & $(0.140)$ \\
\hline \multicolumn{7}{|l|}{ Cooperative traits } \\
\hline \multirow[t]{2}{*}{ Other } & -0.225 & -0.0690 & 0.156 & $-0.285^{*}$ & -0.124 & 0.161 \\
\hline & $(0.177)$ & $(0.209)$ & $(0.286)$ & $(0.164)$ & $(0.177)$ & $(0.190)$ \\
\hline \multirow[t]{2}{*}{ Conditional Cooperators } & -0.0815 & -0.136 & -0.0549 & -0.101 & -0.223 & -0.122 \\
\hline & $(0.168)$ & $(0.226)$ & $(0.319)$ & $(0.170)$ & $(0.194)$ & $(0.199)$ \\
\hline \multirow[t]{2}{*}{ Low Unconditional Cooperators } & $-0.557^{* *}$ & $-0.589^{* *}$ & -0.0321 & $-0.506^{* * *}$ & $-0.485^{* *}$ & 0.0210 \\
\hline & $(0.212)$ & $(0.254)$ & $(0.334)$ & $(0.190)$ & $(0.200)$ & $(0.213)$ \\
\hline \multirow[t]{2}{*}{ Medium Unconditional Cooperators } & $-0.541^{* * *}$ & $-0.468^{*}$ & 0.0728 & $-0.545^{* * *}$ & $-0.510^{* *}$ & 0.0350 \\
\hline & $(0.170)$ & $(0.257)$ & $(0.299)$ & $(0.180)$ & $(0.216)$ & $(0.213)$ \\
\hline \multirow[t]{2}{*}{ Constant } & $3.194^{* * *}$ & $3.618^{* * *}$ & 0.424 & $3.565^{* * *}$ & $3.133^{* * *}$ & -0.432 \\
\hline & $(0.417)$ & $(0.382)$ & $(0.414)$ & $(0.446)$ & $(0.430)$ & $(0.546)$ \\
\hline Commune fixed effects & $\sqrt{ }$ & $\sqrt{ }$ & $\sqrt{ }$ & & & \\
\hline Village fixed effects & & & & $\sqrt{ }$ & $\sqrt{ }$ & $\sqrt{ }$ \\
\hline Risk aversion & $\sqrt{ }$ & $\sqrt{ }$ & $\sqrt{ }$ & $\sqrt{ }$ & $\sqrt{ }$ & $\sqrt{ }$ \\
\hline Socio-demographics & $\sqrt{ }$ & $\sqrt{ }$ & $\sqrt{ }$ & $\sqrt{ }$ & $\sqrt{ }$ & $\sqrt{ }$ \\
\hline Observations & 281 & 281 & 281 & 281 & 281 & 281 \\
\hline$R^{2}$ & 0.108 & 0.099 & 0.013 & 0.203 & 0.255 & 0.162 \\
\hline Adjusted $R^{2}$ & 0.068 & 0.059 & -0.031 & 0.107 & 0.165 & 0.061 \\
\hline
\end{tabular}

Standard errors in parentheses

${ }^{*} p<.10,{ }^{* *} p<.05,{ }^{* * *} p<.01$

\subsubsection{Discussions and conclusion}

The provision of public goods can be fragile if it is based on voluntary contributions by users, as some users may be tempted to underprovide or free ride. We explore with artefactual field experiments under which conditions users vote for institutions that set rules that govern the contribution to public goods. Our results show that a significant share of subjects vote for costly institutions even if they are costly and not necessarily efficient. We find broad support for both a minimum contribution level (a tax system) and also stricter enforcement if it increases the chances of detection for those who underprovide. These findings are in line with other experimental work which find that users choose to implement an institutional setting, even if costly, compared to the voluntary contribution mechanism (Dannenberg et al., 2019; Kamei et al., 2015; Markussen et al., 2014; Sutter et al., 2010). 
Accordingly, we also observe that the average contribution is higher when institutions are in place, suggesting that they do indeed improve cooperation. In the minimum contribution regime, where each subject has to contribute at least half of the endowment to the public good, the majority of them still contributes more than the minimum requirement, suggesting that the tax regime does not crowd out the voluntary contribution beyond the required minimum level. When it comes to the sanctioning institutions, the average contribution in the strict enforcement mechanism is higher than that in the weak enforcement mechanism.

Furthermore, we also analyze effects of individual preferences, wealth and experienced scarcity on the probability of subjects voting for the costly institutions. In both the tax system and the strict enforcement mechanism, we find that greater exposure to water scarcity in the past increases the likelihood of the subjects voting for the institutions. We also consider effects of risk preference and trust on voting behavior, but we do not find any significant effects. We find that wealth has different impacts on the likelihood of subjects supporting the costly institutions, depending on whether the wealth is related to on-farm or off-farm livelihood options. The subjects who possess wealth that can be accumulated through off-farm activities such as fishing and remittance are less likely to support the costly institutions, as compared to those who own wealth that is related directly to farming. The reason could be because those who can earn offfarm income (which can serve as a buffering capacity in the face of scarcity and shock) depend less on farming, and thus find that the support of institution is less important.

Previous studies have shown that the informal institution which is enforced through social norms may collapse in the wake of scarcity, e.g. due to climate change (Nhim et al., 2019; Richter et al., 2013). While our experiments are artefactual, the institutional settings that are featured in our games resemble the type of institution available in the field settings of the study area. Our findings demonstrate that the alternative institution such as the formal institution could be supported, even if they are not welfare-enhancing. This finding suggests that fairness, rather than efficiency, are important element when it comes to supporting institutions (Kosfeld et al., 2009). Finally, individuals are more inclined to vote for such institutions when they were exposed to resource scarcity in the past. This finding may give a bit of hope about the effects of climate change which tends to exacerbate scarcity, as it may also trigger institutional responses that can ameliorate those climate impacts. 


\section{Authors' contribution}

T.N., A.R., and E.S. designed the research. T.N. and E.S. carried out the field experiments. T.N. performed the analysis. All authors contributed to writing the final manuscript.

\section{Acknowledgments}

T.N. gratefully acknowledges the funding from the Center for Khmer Studies, project number 900-185. 


\section{References}

Agrawal, A. (2008). The Role of Local Institutions in Adaptation to Climate Change.

Bastakoti, R. C., \& Shivakoti, G. P. (2012). Rules and collective action: an institutional analysis of the performance of irrigation systems in Nepal. Journal of Institutional Economics, 8(2), 225-246. https://doi.org/10.1017/S1744137411000452

Bisaro, A., \& Hinkel, J. (2016). Governance of social dilemmas in climate change adaptation. Nature Climate Change, 6(4), 354-359.

Blanco, E., Lopez, M. C., \& Villamayor-Tomas, S. (2015). Exogenous degradation in the commons: Field experimental evidence. Ecological Economics, 120, 430-439. https://doi.org/10.1016/j.ecolecon.2015.03.028

Burton-Chellew, M. N., \& West, S. A. (2013). Prosocial preferences do not explain human cooperation in public-goods games. Proceedings of the National Academy of Sciences, $110(1), 216-221$.

Chea, C. (2010). The Local Governance of Common Pool Resources: The Case of Irrigation Water in Cambodia. In CDRI Working Paper Series No. 47.

Chem, P., Kim, S. S., \& Khiev, D. (2010). Empirical evidence of irrigation management in the Tonle Sap Basin: Issues and challenges. In CDRI Working Paper Series No. 48.

Dal Bo, P., Foster, A., \& Putterman, L. (2010). Institutions and Behavior: Experimental Evidence on the Effects of Democracy. 100(5), 2205-2229.

Dannenberg, A., \& Gallier, C. (2019). The choice of institutions to solve cooperation problems: a survey of experimental research. Experimental Economics. https://doi.org/10.1007/s10683-019-09629-8

Dannenberg, A., Haita-Falah, C., \& Zitzelsberger, S. (2019). Voting on the threat of exclusion in a public goods experiment. Experimental Economics. https://doi.org/10.1007/s10683019-09609-y

Diepart, J.-C. (Ed.). (2015). Learning for Resilience: Insights from Cambodia's Rural Communities. The Learning Institute.

Ertan, A., Page, T., \& Putterman, L. (2009). Who to punish? Individual decisions and majority rule in mitigating the free rider problem. European Economic Review, 53(5), 495-511. https://doi.org/10.1016/j.euroecorev.2008.09.007

Fallucchi, F., Luccasen, R. A., \& Turocy, T. L. (2018). Identifying discrete behavioural types: a re-analysis of public goods game contributions by hierarchical clustering. Journal of the Economic Science Association, 5(2), 238-254. https://doi.org/10.1007/s40881-018-0060- 
Fehr, E., \& Fischbacher, U. (2003). The nature of human altruism. Nature, 425(6960), 785.

Feld, L. P., \& Tyran, J.-R. (2002). Tax Evasion and Voting: An Experimental Analysis. Kyklos, 55(2), 197-221. https://doi.org/10.1111/1467-6435.00183

Finkbeiner, E. M., Micheli, F., Saenz-Arroyo, A., Vazquez-Vera, L., Perafan, C. A., \& Cárdenas, J. C. (2018). Local response to global uncertainty: Insights from experimental economics in small-scale fisheries. Global Environmental Change, 48(October 2017), 151-157. https://doi.org/10.1016/j.gloenvcha.2017.11.010

Gallier, C. (2020). Democracy and Compliance in Public Goods Games. European Economic Review, 121, 103346. https://doi.org/10.2139/ssrn.3057268

Gneezy, U., \& Potters, J. (1997). An experiment on risk taking and evaluation periods.

Quarterly Journal of Economics, 112(2), 631-645.

https://doi.org/10.1162/003355397555217

Grossman, H. I., \& Mendoza, J. (2003). Scarcity and appropriative competition. European Journal of Political Economy, 19(4), 747-758.

Haddeland, I., Heinke, J., Biemans, H., Eisner, S., Flörke, M., Hanasaki, N., Konzmann, M., Ludwig, F., Masaki, Y., Schewe, J., Stacke, T., Tessler, Z. D., Wada, Y., \& Wisser, D. (2014). Global water resources affected by human interventions and climate change. Proceedings of the National Academy of Sciences, 111(9), 3251-3256.

Jaeger, W. K., Amos, A., Bigelow, D. P., Chang, H., Conklin, D. R., Haggerty, R., Langpap, C., Moore, K., Mote, P. W., Nolin, A. W., Plantinga, A. J., Schwartz, C. L., Tullos, D., \& Turner, D. P. (2017). Finding water scarcity amid abundance using human-natural system models. Proceedings of the National Academy of Sciences, 114(45), 11884-11889.

Janssen, M. A., Lindahl, T., \& Murphy, J. J. (2015). Advancing the understanding of behavior in social-ecological systems: results from lab and field experiments. Ecology and Society, 20(4). https://doi.org/10.5751/ES-08097-200434

Kamei, K., Putterman, L., \& Tyran, J. R. (2015). State or nature? Endogenous formal versus informal sanctions in the voluntary provision of public goods. Experimental Economics, 18(1), 38-65. https://doi.org/10.1007/s10683-014-9405-0

Kocher, M. G., Martinsson, P., Persson, E., \& Wang, X. (2016). Is there a hidden cost of imposing a minimum contribution level for public good contributions? Journal of Economic Psychology, 56, 74-84. https://doi.org/10.1016/j.joep.2016.05.007

Kosfeld, M., Okada, A., \& Riedl, A. (2009). Institution formation in public goods games. American Economic Review, 99(4), 1335-1355. https://doi.org/10.1257/aer.99.4.1335 
Kroll, S., Cherry, T. L., \& Shogren, J. F. (2007). Voting, punishment, and public goods. Economic Inquiry, 45(3), 557-570. https://doi.org/10.1111/j.1465-7295.2007.00028.x

Ledyard, J. O. (1995). Public goods: A survey of experimental research. In R. A. Kagel, J. (Ed.). Princeton University Press, Princeton.

Mak, S. (2017). Water Governance in Cambodia: From Centralized Water Governance to Farmer Water User Community. Resources, 6(3), 44.

Markussen, T., Putterman, L., \& Tyran, J. R. (2014). Self-organization for collective action: An experimental study of voting on sanction regimes. Review of Economic Studies, 81(1), 301-324. https://doi.org/10.1093/restud/rdt022

Martinsson, P., \& Persson, E. (2019). Public Goods and Minimum Provision Levels: Does the Institutional Formation Affect Cooperation? Scandinavian Journal of Economics, 000(0), 1-27. https://doi.org/10.1111/sjoe.12321

Nhim, T., Richter, A., \& Zhu, X. (2019). The resilience of social norms of cooperation under resource scarcity and inequality - An agent-based model on sharing water over two harvesting seasons. Ecological Complexity, 40. https://doi.org/10.1016/j.ecocom.2018.06.001

North, D. C. (1991). Institutions. Journal of Economic Perspectives, 5(1), 97-112.

Osés-Eraso, N., Udina, F., \& Viladrich-Grau, M. (2008). Environmental versus human-induced scarcity in the commons: do they trigger the same response? Environmental and Resource Economics, 40(4), 529-550. https://doi.org/10.1007/s10640-007-9168-6

Osés-Eraso, N., \& Viladrich-Grau, M. (2007). On the sustainability of common property resources. Journal of Environmental Economics and Management, 53(3), 393-410.

Ostrom, E. (1990). Governing the commons: the evolution of institutions for collective action. Cambridge University Press.

Ostrom, E. (2000). Collective Action and the Evolution of Social Norms. Journal of Economic Perspectives, 14(3), 137-158.

Ostrom, E. (2009). A general framework for analyzing sustainability of social-ecological systems. Science, 325(5939), 419-422.

Ostrom, E., Burger, J., Field, C. B., Norgaard, R. B., \& Policansky, D. (1999). Revisiting the commons: Local lessons, global challenges. Science, 284(5412), 278-282. https://doi.org/10.1126/science.284.5412.278

Ostrom, E., \& Gardner, R. (1993). Coping with asymmetries in the commons: self-governing irrigation systems can work. The Journal of Economic Perspectives, 7(4), 93-112. https://doi.org/10.1257/jep.7.4.93 
Pfaff, A., Vélez, M. A., Ramos, P. A., \& Molina, A. (2015). Framed field experiment on resource scarcity \& extraction: path-dependent generosity within sequential water appropriation. Ecological Economics, 120, 416-429.

Richter, A., Schuch, E., \& Nhim, T. (2020). Cooperation under uncertainty in natural resource management. https://doi.org/10.17605/OSF.IO/U3GC8.

Richter, A., van Soest, D., \& Grasman, J. (2013). Contagious cooperation, temptation, and ecosystem collapse. Journal of Environmental Economics and Management, 66(1), 141158.

Sam, S., \& Pech, S. (Eds.). (2015). Climate Change and Water Governance in Cambodia: Challenge and Perspectives for Water Security and Climate Change in Selected Catchments. CDRI - Cambodia Development Resource Institute.

Schewe, J., Heinke, J., Gerten, D., Haddeland, I., Arnell, N. W., Clark, D. B., Dankers, R., Eisner, S., Fekete, B. M., Colón-González, F. J., Gosling, S. N., Kim, H., Liu, X., Masaki, Y., Portmann, F. T., Satoh, Y., Stacke, T., Tang, Q., Wada, Y., ... Kabat, P. (2014). Multimodel assessment of water scarcity under climate change. Proceedings of the National Academy of Sciences, 111(9), 3245-3250.

Schill, C., Wijermans, N., Schlüter, M., \& Lindahl, T. (2016). Cooperation is not enough Exploring social-ecological micro-foundations for sustainable common-pool resource use. PLoS ONE, 11(8). https://doi.org/10.1371/journal.pone.0157796

Schlüter, M., Tavoni, A., \& Levin, S. (2016). Robustness of norm-driven cooperation in the commons. Proc. R. Soc. B, 283(1822).

Schuch, E., Dirks, S.,Nhim, T., \& Richter, A. (2021). Cooperation under social and strategic uncertainty: The role of risk and social capital in rural Cambodia. Journal of Behavioral and Experimental Economics (90), https://doi.org/10.1016/j.socec.2020.101642

Shreedhar, G., Tavoni, A., \& Marchiori, C. (2019). Monitoring and punishment networks in a common-pool resource dilemma. Environment and Development Economics, 1-29. https://doi.org/10.1017/S1355770X19000457

Sutter, M., Haigner, S., \& Kocher, M. G. (2010). Choosing the carrot or the stick? Endogenous institutional choice in social dilemma situations. Review of Economic Studies, 77(4), 1540-1566. https://doi.org/10.1111/j.1467-937X.2010.00608.x

Sutter, M., \& Weck-Hannemann, H. (2003). On the effects of asymmetric and endogenous taxation in experimental public goods games. In Economics Letters (Vol. 79, Issue 1, pp. 59-67). https://doi.org/10.1016/S0165-1765(02)00288-4

Sutter, M., \& Weck-Hannemann, H. (2004). An experimental test of the public-goods 
crowding-out hypothesis when taxation is endogenous. FinanzArchiv: Public Finance Analysis, 60(1), 94-110.

Tavoni, A., Schlüter, M., \& Levin, S. (2012). The survival of the conformist: Social pressure and renewable resource management. Journal of Theoretical Biology, 299, 152-161. https://doi.org/10.1016/j.jtbi.2011.07.003

Tyran, J. R., \& Feld, L. P. (2006). Achieving compliance when legal sanctions are nondeterrent. Scandinavian Journal of Economics, 108(1), 135-156. https://doi.org/10.1111/j.1467-9442.2006.00444.x

Vollan, B., Landmann, A., Zhou, Y., Hu, B., \& Herrmann-Pillath, C. (2017). Cooperation and authoritarian values: An experimental study in China. European Economic Review, 93, 90-105. https://doi.org/10.1016/j.euroecorev.2017.01.007

Yeboah-Assiamah, E., Muller, K., \& Domfeh, K. A. (2017). Institutional assessment in natural resource governance: A conceptual overview. Forest Policy and Economics, 74, 1-12. https://doi.org/10.1016/j.forpol.2016.10.006 


\section{Chapter 6-Synthesis}

\subsection{Introduction}

Worldwide, water governance is facing pressing challenge due to climate change (Haddeland et al. 2014; Elliott et al. 2014; Schewe et al. 2014; Jaeger et al. 2017; Veldkamp et al. 2017). A small-scale irrigation system is thus no exception in this regard. An irrigation system is part of a social-ecological system, in which the interactions between users, the resource system, and the embedded institutions are complex and nonlinear (Ostrom 2009; Liu et al. 2007; Wada et al. 2017). At the heart of its management is understanding how human behavior co-evolves with its biophysical system under various institutional arrangements and resource conditions (Schill et al. 2019; Yu et al. 2015; Anderies et al. 2004). The sustainability of such a system depends largely on the self-organizing capacity of resource users for collective action such as cooperation (Anderies et al. 2004; Ostrom 2009). This thesis uses both evolutionary agentbased model and field experiments, to analyze how changing resource conditions that may bring about scarcity interact with resource users' attributes and their embedded institutions and cooperative arrangements.

\subsection{Overview of results and discussions}

The role of social norms and informal institutions in maintaining cooperative resource management is prevalent in small self-governing communities (Ostrom 1990). The ability of informal institutions to adapt is especially important in the face of climate change (Adger et al. 2005). System productivity has been identified as a key variable that is associated with level of cooperation and success of self-governance (Ostrom 2009). The interaction between changes in scarcity and cooperation, however, remains unclear. While a strand of literature suggests that scarcity undermines cooperation (Grossman and Mendoza 2003; Blanco et al. 2015; Richter et al. 2013; Pfaff et al. 2015), another strand posits that scarcity may indeed increase cooperation (Osés-Eraso et al. 2008; Schlüter et al. 2016; Nie et al. 2020). This thesis investigates how the effect of resource scarcity on cooperation is mediated by contextual variables in the field, such as inequality, cooperative orientation and individual experience, and ultimately affect cooperation. Understanding how scarcity and cooperation interacts thus may enhance our understanding of how self-governance will be able to cope with climate change. 
Findings in Chapter 2 show that social norms of cooperation may collapse in times of resource scarcity, and the dynamics are mediated by inequality and heterogeneity of users. I find that under increasing scarcity, highly efficient agents who possess high land endowment are more successful, taking larger proportion of resource, leaving most others with an increasingly smaller share of the resource. This finding is in line with model results by Mirza et al. (2019). I find that this mechanism is particularly strong if social capital and peer enforcement is weak. Under high scarcity, individual costs of cooperating by restraining harvesting behavior and enforcing the norms are relatively high. As shown in Chapter 2, inequality interacts with scarcity, and its effect on cooperation depends on how the ability to discipline others with peer enforcement. Thus, to understand better how climate change may affect the resilience of cooperative resource arrangements, the interactions between inequality and the role of institution in moderating it needs to be considered (Tavoni et al. 2011; Mirza et al. 2019).

Chapter 3 investigates the role of scarcity further by looking at how it interacts with institutions, and affect cooperation among resource users who face coupled collective action problems in common-pool resource extraction as well as in infrastructure investment giving rise to a public goods problem. Field experiments conducted in Cambodia (see Chapter 5) suggest that existing institutions may respond to scarcity and vice versa. If the community has a functioning water system initially and a high level of cooperation, prosperity can be created, which facilitates further investments in water infrastructure, fostering cooperation further. If the community has a poorly functioning water system initially featuring resource scarcity, cooperation is relatively costly, undermining investments in water infrastructure, potentially giving rise to an institutional trap. Model results confirm these observations from field evidence, but also show that if social capital is stronger, the institutional trap can be overcome, and prosperity can be created. These findings are in line with what has been shown for the case of a forest selfgovernance in Ethiopia (Rustagi et al. 2010). Findings in Chapter 3 explain further that institutional arrangements may respond to scarcity due to the presence of conditional cooperators. Under high scarcity, violating norms is lucrative, and the cost of doing so depends on the cooperative attitude of others. Conditional cooperators respond to their social environment accordingly depending on whether cooperation is common or rare (Traxler and Spichtig 2011), and in particular the level of contributions by others (Frey and Meier 2004). Chapter 4 takes a step further by studying the evolution of cooperation between two communities who share water as a common-pool resource and infrastructure as a public good. 
The results show that cooperation cannot be maintained when the public good is present, unless a reputation mechanism is in place. Further, the model results show that higher system productivity resulted from increased cooperation in the upstream community leads to more cooperation in the downstream community as well as higher welfare overall, but only in the presence of social interactions between communities. If cooperation in the downstream is enhanced, however, it does not translate into more cooperation in the upstream. Previous studies considering the combined problem of public good provision and common-pool resource extraction conclude that the presence of public goods could serve as economic interdependency and a unified purpose that may thus allow cooperation to sustain (Solstad and Brekke 2011; Botelho et al. 2015). When the interactions are studied at multiple levels (between and within communities), as in Chapter 4, I find that this is not the case. This chapter highlights the tension to cooperate within and between communities of resource users, and that cooperation in both communities depends on social structure and characteristics that enable learning within and across communities.

Chapter 5 is based on lab-in-the-field experiments in Cambodia, in which real field users of common-pool resources face two institutional choices: one resembles their existing informal institution in which the enforcement is weak, while the other resembles a more centralized enforcement mechanism in which enforcement is strict but costly. In the latter, this resembles a governance system of a farmer organization, the so-called Farmer Water User Community, which self-governs the small-scale irrigation system in the study area. Findings from Chapter 5 show a broad support for the costly institution, and even more so if players had been exposed to resource scarcity in the past, implying that individual experience about prior exposure to scarcity may have a connection with support for management institutions.

One of the main objectives of the thesis is to disentangle the multiplicity of interactions between scarcity and contextual factors and its effects on cooperation through the lens of institutions. Combining evolutionary agent-based model and field experiments, I find that scarcity may interact with key variables in the field such as inequality (Chapter 2), individual experience (Chapter 5) and preferences (Chapter 3), to affect cooperation. Methodologically, the interacting effects could well explain why a single approach to the study of scarcity and cooperation may not provide a complete picture of its rich dynamics in a small-scale socialecological system. This thesis brings stylized facts generated through findings from field experiments, and tests them in the modelling setting to gain deeper understanding how social 
dynamics, institutions, and scarcity affect each other (Chapter 3). At the same time, theoretical results from the model were also tested in the field (Chapter 5). Through this combination of approaches, thematically, overall the thesis shows that scarcity undermines cooperation, especially when resource users are heterogeneous (Chapter $2 \& 3$ ). This s especially the case when agents are assumed to be unconditional cooperators (Chapter 2), and that the institution may interact with scarcity by creating a path-dependency-if the system has a poorly functioning system which features scarcity and low cooperation initially, it remains trapped and is unable to evolve (Chapter 3). However, both field evidence and the modeling results suggest that conditional cooperation could be beneficial for institution to prosper by further facilitating cooperation under scarcity.

Another objective of the thesis is to understand better the dynamics of coupled social dilemmas among resource users who jointly harvest water as a common-pool resource, while at the same time rely on joint effort for its production, such as investing in a shared water infrastructure. Previous studies establish that coupling the public good to cooperative resource extraction can foster cooperation, though some individuals may take a larger share in providing (Botelho et al. 2015; Solstad and Brekke 2011). An open question is how or why the public good is provided by some agents in the first place. The model results (Chapter 3) suggest that if the community is largely comprised of low contributing agents in the first place, and faces initial scarcity, the system cannot evolve and remains trapped in the regime of low contribution to the public good which thus leads to a collapse of cooperation. So if climate change causes scarcity to increase, will resource users be able to join hands and contribute to the public good? I find that if not enough number of agents contribute to the maintenance of the infrastructure, water cannot be stored in the system, leaving everyone worse off. The results suggest that conditional cooperation could be key to overcoming such an institutional trap. Conditional cooperation can mitigate initial scarcity and further facilitate the contribution to the public good and hence cooperation in the common pool resource. Chapter 4 , however, identifies a further challenge if the resource is shared between two communities. Even if the public good is provided from a joint effort by members in both communities, cooperation in one community does not directly spread to the other. This is especially true when one community has an advantage over the other in terms of resource access, which is typically the case in a small-scale irrigation system. For a self-governing system between communities, contextual variables such as social capital and social networks could play a role in promoting cooperation. 
Overall, the findings from this thesis have two implications for researchers and practitioners. First, when evaluating the impacts of climate change on the self-governance of a common resources, it is important to consider institutional configurations which may respond to scarcity, potentially aggravating or moderating scarcity, depending on characteristics and structure of the social system. Second, the findings caution against considering social dilemma in common pool resources and public goods management in isolation, as the unfolding dynamics emerged from the interaction between the two may be very different than if considered alone. Further, the findings suggest that having experienced scarcity in the past may create public support for institutional change, which is observed in the field work, but have not been formalized in a model yet.

\subsection{Limitations, and future research}

While part of the model assumptions and results in this thesis are informed by field experiments, many findings in this thesis are theoretically grounded. The lack of empirical grounding of the model work limits predictions about what we will observe in the field. The next step would be to bring the model predictions and test them in the field, by, for example, conducting experiments. Previous experiments focus on either the role of inequality and heterogeneity of users or the role of scarcity on cooperation, but rarely both combined. Further, while theoretical models on coupled social dilemmas of common-pool resource and public good are rarely studied, potentially due to its complexity, the field experiments that incorporate the interactions between the two also remain unexplored. To date, Botelho et al. (2015) have tested such setting in the lab by simplifying the games to a great extent. Conducting such experiments in the field with a similar setup would thus enhance our understanding of the nature of the complex social and resource dynamics in the field. Finally, while the experiments in this thesis (Chapter 5) mimic the institutional settings that may resemble the type of institution available in the study area, the roles of scarcity are not directly incorporated in the games. An experimental setting that allows for the analysis that can reveal to what extent and which types of institution the real resource users may support under experimentally-controlled scarcity would be an exciting next step to inform sustainable resource management under climate change. 


\section{References}

Adger, W. Neil, Terry P. Hughes, Carl Folke, Stephen R. Carpenter, and Johan Rockström. 2005. “Social-Ecological Resilience to Coastal Disasters.” Science 309 ((5737): 103639. https://doi.org/10.4324/9781351201117-18.

Anderies, John M, Marco a Janssen, and Elinor Ostrom. 2004. "A Framework to Analyze the Robustness of Social-Ecological Systems from an Institutional Perspective.” Ecology and Society 9 (1): 1-18. https://doi.org/18.

Blanco, Esther, Maria Claudia Lopez, and Sergio Villamayor-Tomas. 2015. "Exogenous Degradation in the Commons: Field Experimental Evidence." Ecological Economics 120: 430-39. https://doi.org/10.1016/j.ecolecon.2015.03.028.

Botelho, Anabela, Ariel Dinar, Lígia M.Costa Pinto, and Amnon Rapoport. 2015. "Promoting Cooperation in Resource Dilemmas: Theoretical Predictions and Experimental Evidence." Journal of Behavioral and Experimental Economics 54: 40-49. https://doi.org/10.1016/j.socec.2014.11.007.

Elliott, Joshua, Delphine Deryng, Christoph Müller, Katja Frieler, Markus Konzmann, Dieter Gerten, Michael Glotter, et al. 2014. "Constraints and Potentials of Future Irrigation Water Availability on Agricultural Production under Climate Change." Proceedings of the National Academy of Sciences 111 (9): 3239-44.

Frey, Bruno S., and Stephan Meier. 2004. "Social Comparisons and Pro-Social Behavior:

Testing Conditional Cooperation in a Field Experiment." American Economic Review 94 (5): 1717-22. https://doi.org/10.1257/0002828043052187.

Grossman, Herschel I., and Juan Mendoza. 2003. "Scarcity and Appropriative Competition." European Journal of Political Economy 19 (4): 747-58.

Haddeland, Ingjerd, Jens Heinke, Hester Biemans, Stephanie Eisner, Martina Flörke, Naota Hanasaki, Markus Konzmann, et al. 2014. "Global Water Resources Affected by Human Interventions and Climate Change." Proceedings of the National Academy of Sciences 111 (9): 3251-56.

Jaeger, William K., Adell Amos, Daniel P. Bigelow, Heejun Chang, David R. Conklin, Roy Haggerty, Christian Langpap, et al. 2017. "Finding Water Scarcity amid Abundance Using Human-Natural System Models." Proceedings of the National Academy of Sciences 114 (45): 11884-89.

Liu, Jianguo, Thomas Dietz, S. Carpenter, Marina Alberti, Carl Folke, Emilio Moran, Alice N 
Pell, et al. 2007. "Complexity of Coupled Human and Natural Systems." Science 317 (5844): 1513-16.

Mirza, M. Usman, Andries Richter, Egbert H. van Nes, and Marten Scheffer. 2019.

“Technology Driven Inequality Leads to Poverty and Resource Depletion.” Ecological Economics 160 (February): 215-26. https://doi.org/10.1016/j.ecolecon.2019.02.015.

Nie, Zihan, Xiaojun Yang, and Qin Tu. 2020. "Resource Scarcity and Cooperation : Evidence from a Gravity Irrigation System in China.” World Development 135: 105035. https://doi.org/10.1016/j.worlddev.2020.105035.

Osés-Eraso, Nuria, Frederic Udina, and Montserrat Viladrich-Grau. 2008. "Environmental versus Human-Induced Scarcity in the Commons: Do They Trigger the Same Response?" Environmental and Resource Economics 40 (4): 529-50. https://doi.org/10.1007/s10640-007-9168-6.

Ostrom, Elinor. 1990. Governing the Commons: The Evolution of Institutions for Collective Action. Cambridge University Press.

_. 2009. "A General Framework for Analyzing Sustainability of Social-Ecological Systems." Science 325 (5939): 419-22.

Pfaff, Alexander, Maria Alejandra Vélez, Pablo Andres Ramos, and Adriana Molina. 2015. "Framed Field Experiment on Resource Scarcity \& Extraction: Path-Dependent Generosity within Sequential Water Appropriation.” Ecological Economics 120: 416-29. Richter, A., D. van Soest, and J. Grasman. 2013. "Contagious Cooperation, Temptation, and Ecosystem Collapse.” Journal of Environmental Economics and Management 66 (1): $141-58$.

Rustagi, Devesh, Engel Stefanie, and Michael Kosfeld. 2010. "Conditional Cooperation and Costly Monitoring Explain Success in Forest Commons Management.” Science 330 (6006): 961-65. https://doi.org/10.1126/science.1193649.

Schewe, Jacob, Jens Heinke, Dieter Gerten, Ingjerd Haddeland, Nigel W. Arnell, Douglas B. Clark, Rutger Dankers, et al. 2014. "Multimodel Assessment of Water Scarcity under Climate Change." Proceedings of the National Academy of Sciences 111 (9): 3245-50.

Schill, Caroline, John M. Anderies, Therese Lindahl, Carl Folke, Stephen Polasky, Juan Camilo Cárdenas, Anne Sophie Crépin, Marco A. Janssen, Jon Norberg, and Maja Schlüter. 2019. "A More Dynamic Understanding of Human Behaviour for the Anthropocene.” Nature Sustainability 2 (12): 1075-82. https://doi.org/10.1038/s41893019-0419-7.

Schlüter, Maja, Alessandro Tavoni, and Simon Levin. 2016. "Robustness of Norm-Driven 
Cooperation in the Commons." Proc. R. Soc. B 283 (1822).

Solstad, Jan Tore, and Kjell Arne Brekke. 2011. "Does the Existence of a Public Good Enhance Cooperation among Users of Common-Pool Resources?” Land Economics 87 (2): 335-45. https://doi.org/10.1353/lde.2011.0023.

Tavoni, Alessandro, Astrid Dannenberg, Giorgos Kallis, and Andreas Löschel. 2011.

"Inequality, Communication, and the Avoidance of Disastrous Climate Change in a Public Goods Game." Proceedings of the National Academy of Sciences of the United States of America 108 (29): 11825-29. https://doi.org/10.1073/pnas.1102493108.

Traxler, Christian, and Mathias Spichtig. 2011. "Social Norms and the Indirect Evolution of Conditional Cooperation." Journal of Economics/ Zeitschrift Fur Nationalokonomie 102 (3): 237-62. https://doi.org/10.1007/s00712-010-0173-9.

Veldkamp, T.I.E., Y. Wada, J.C.J.H. Aerts, P. Döll, S. N. Gosling, J. Liu, Y. Masaki, et al. 2017. "Water Scarcity Hotspots Travel Downstream Due to Human Interventions in the 20th and 21st Century." Nature Communications 8.

https://doi.org/10.1038/ncomms15697.

Wada, Yoshihide, Marc F P Bierkens, Ad De Roo, Paul A Dirmeyer, James S Famiglietti, Naota Hanasaki, Megan Konar, Junguo Liu, and Hannes Müller Schmied. 2017. "Human-Water Interface in Hydrological Modelling: Current Status and Future Directions.” Earth Syst. Sci 215194: 4169-93. https://doi.org/10.5194/hess-21-41692017.

Yu, David J, Murad R Qubbaj, Rachata Muneepeerakul, John M Anderies, and Rimjhim M Aggarwal. 2015. "Effect of Infrastructure Design on Commons Dilemmas in SocialEcological System Dynamics." Proceedings of the National Academy of Sciences of the United States of America 112 (43): 1410688112-. https://doi.org/10.1073/pnas.1410688112. 


\section{Summary}

Worldwide, water governance is facing pressing challenges due to climate change. A smallscale irrigation system is thus no exception in this regard. An irrigation system is part of a social-ecological system (SES), in which the interactions between the subsystems are complex and nonlinear. The sustainability of such a system depends largely on the self-organizing capacity of resource users for collective action, for example by cooperating. This thesis focuses on a small-scale SES, namely an irrigation system which consists of the resource (water), water users (farmers), and the governance system (informal and formal institutions). I use evolutionary agent-based models and field experiments to analyzes under what conditions, and with which institutional mechanisms cooperative arrangements can be established and maintained under climate change.

This thesis consists of six chapters. In Chapter 1 I describes the general societal and academic problem which lead to the scientific research questions. Further, I describe the contribution of the thesis to the current literature, and reflect on the research methods used in the thesis. In Chapter 2, I analyse how resource scarcity interacts with inequality and affects the emergence and stability of cooperative arrangements, using an evolutionary agent-based model. Findings in Chapter 2 show that social norms of cooperation may collapse in times of resource scarcity, and the dynamics are mediated by inequality and heterogeneity of users. This is especially the case if social capital and peer enforcement are weak. Thus, to understand better how climate change may affect the resilience of cooperative resource arrangements, the interactions between inequality and the role of institution in moderating scarcity needs to be considered.

Chapter 3 investigates the role of scarcity further by looking at how it interacts with institutions, and affects cooperation among resource users who face coupled collective action problems. In particular, I consider the case where users are confronted with the social dilemma of commonpool resource extraction, as well as the public goods problem of investing in water infrastructure. Field experiments conducted in Cambodia suggest that existing institutions may respond to scarcity and vice versa. If the community has initially a functioning water system and a high level of cooperation, prosperity can be created, which facilitates further investments in water infrastructure, fostering cooperation further. If the community has initially a poorly functioning water system featuring resource scarcity, cooperation is relatively costly, undermining investments in water infrastructure, potentially giving rise to an institutional trap. 
Model results confirm these observations from field evidence, but also show that if social capital is stronger, the institutional trap can be overcome, and prosperity can be created. Findings in Chapter 3 explain further that institutional arrangements may respond to scarcity due to the presence of conditional cooperators. The findings also caution against considering social dilemmas in common pool resources and contribution to public goods in isolation, as the unfolding dynamics emerging from the interactions between the two coupled dilemmas may be very different than if considered alone.

Chapter 4 takes a step further by studying the evolution of cooperation between two communities who share water as a common-pool resource and infrastructure as a public good. The results show that cooperation cannot be maintained when the public good is present, unless a reputation mechanism is in place. Further, the model results show that higher system productivity due to increased cooperation in the upstream community leads to more cooperation in the downstream community as well as higher welfare overall, but only in the presence of social interactions between communities. If cooperation in the downstream is enhanced, however, it does not translate into more cooperation in the upstream. This chapter highlights the tension to cooperate within and between communities of resource users, and that cooperation in both communities depends on social structure and characteristics that enable learning within and across communities.

Chapter 5 is based on lab-in-the-field experiments in Cambodia, in which real field users of common-pool resources face two institutional choices: one resembles their existing informal institution in which the enforcement is weak, while the other resembles a more centralized enforcement mechanism in which enforcement is strict but costly. In the latter, this resembles a governance system of a farmer organization, the so-called Farmer Water User Community, which self-governs the small-scale irrigation system in the study area. Findings from Chapter 5 show a broad support for the costly institution, and even more so if players had been exposed to resource scarcity in the past, implying that individual experience about prior exposure to scarcity may have a connection with support for management institutions. 


\section{SENSE}

Netherlands Research School for the

Socio-Economic and Natural Sciences of the Environment

\section{I P L O M A \\ for specialised PhD training}

The Netherlands research school for the Socio-Economic and Natural Sciences of the Environment (SENSE) declares that

\section{Tum Nhim}

born on $10^{\text {th }}$ December 1987 in Kampot, Cambodia

has successfully fulfilled all requirements of the educational PhD programme of SENSE.

Wageningen, $1^{\text {st }}$ July 2021

Chair of the SENSE board

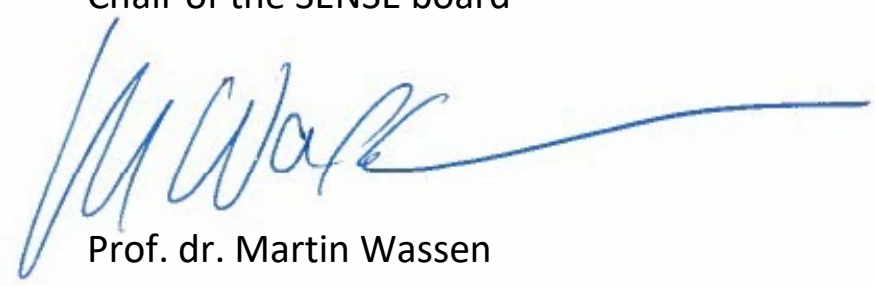

The SENSE Director

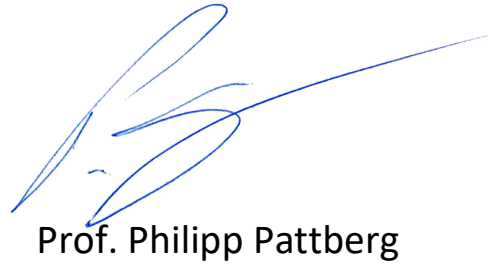

The SENSE Research School has been accredited by the Royal Netherlands Academy of Arts and Sciences (KNAW)

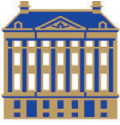

$\begin{array}{llllllllllllllllllllll}K & O & N & I & N & K & L & I & J & K & E & N & E & D & E & R & L & A & N & D & S & E\end{array}$

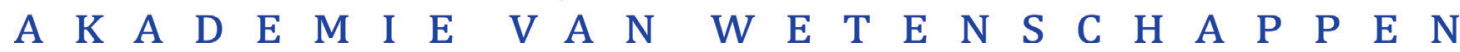




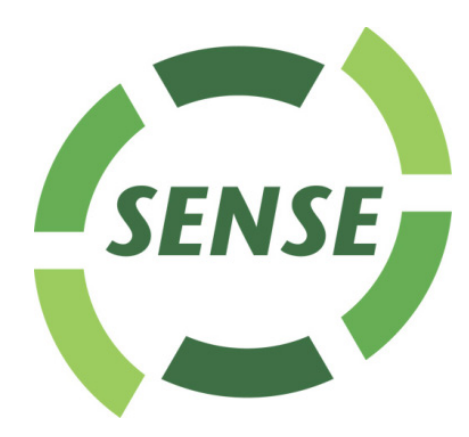

The SENSE Research School declares that Tum Nhim has successfully fulfilled all requirements of the educational PhD programme of SENSE with a work load of 35.4 EC, including the following activities:

\section{SENSE PhD Courses}

- Environmental research in context (2016)

- Research in context activity: 'Development of Grant Research Proposal and Project Management (2019)

- Bayesian Statistics (2016)

o The Art of Modelling (2017)

\section{Other PhD and Advanced MSc Courses}

- Reviewing a scientific paper, Wageningen Graduate School (2016)

- Techniques for writing and presenting scientific paper, Wageningen Graduate School (2016)

- Advance Microeconomics, Wageningen University (2016)

- Economics and Management of Natural Resources, Wageningen University (2016)

- Modeling environmental resilience, CERES, Ecole Normale Supérieure (2016)

- Agent-based modeling for resilience, ESSA/SiLiCo (2017)

\section{External training at a foreign research institute}

- Young Scientists Summer Program, IIASA, Austria (2018)

\section{Oral Presentations}

- The resilience of social norms of cooperation under resource scarcity and inequality - an agent-based model on sharing water over two harvesting seasons, World Conference on Natural Resource Modelling, 6-9 June 2017, Spain, Barcelona

- Endogenous institution and cooperation among farmers and fishers: Experimental evidence from Cambodia. 21st Annual BIOECON Conference, 11-13 September 2019, Wageningen, The Netherlands

SENSE coordinator PhD education

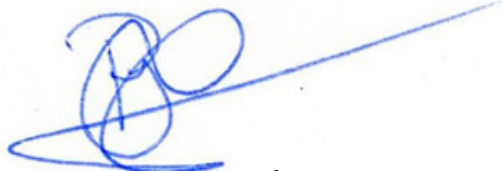

Dr. ir. Peter Vermeulen 
The research in this thesis received funding from the European Commission through the Alfabet project under the EU Erasmus Mundus Program. Research visits at the International Institute for Applied Systems Analysis (IIASA), Austria, have been financially supported by NWO. Research fieldworks in Cambodia received funding from the Center for Khmer Studies, project number 900-185.

Cover photo and design by Tum Nhim 
